\title{
Supramolecular Iron Metallocubanes Exhibiting Site-Selective Thermal and Light-Induced Spin-Crossover
}

\author{
Izar Capel Berdiell, ${ }^{a}$ Tim Hochdörffer, ${ }^{\mathrm{b}}$ Cédric Desplanches, ${ }^{\mathrm{c}}$ Rafal Kulmaczewski, ${ }^{\mathrm{a}}$ \\ Namrah Shahid, ${ }^{\mathrm{a}}$ Juliusz A. Wolny, ${ }^{\mathrm{b}}$ Stuart L. Warriner, ${ }^{\mathrm{a}}$ Oscar Cespedes, ${ }^{\mathrm{d}}$ Volker Schünemann, ${ }^{\mathrm{b}}$ \\ Guillaume Chastanet ${ }^{*, \mathrm{c}}$ and Malcolm A. Halcrow*,a \\ ${ }^{a}$ School of Chemistry, University of Leeds, Woodhouse Lane, Leeds LS2 9JT, \\ United Kingdom. \\ E-mail: m.a.halcrow@leeds.ac.uk \\ ${ }^{b}$ Department of Physics, Technical University of Kaiserslautern, Erwin Schrödinger Str. 46, \\ D-67663 Kaiserslautern, Germany. \\ ${ }^{c}$ CNRS, Univ. Bordeaux, ICMCB, UMR 5026, F-33600 Pessac, France. \\ E-mail: chastanet@icmcb-bordeaux.cnrs.fr \\ ${ }^{d}$ School of Physics and Astronomy, EC Stoner Building, University of Leeds, Leeds, UK LS2 9JT.
}

\section{Supporting Information}


Table S1 Experimental data for the crystal structure determinations of 1.6MeCN. S5

Table S2 Experimental data for the crystal structure determinations of $\mathbf{2} \cdot x \mathrm{MeCN} \cdot y\left(\mathrm{C}_{2} \mathrm{H}_{5}\right)_{2} \mathrm{O} . \quad$ S6

$\begin{array}{lll}\text { Table S3 } & \text { Experimental data for the other crystal structure determinations. } & \text { S7 }\end{array}$

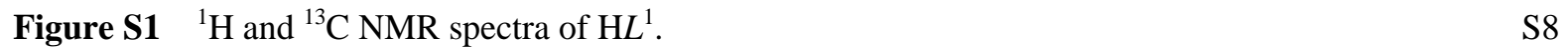

Figure S2 ${ }^{1} \mathrm{H}$ and ${ }^{13} \mathrm{C}$ NMR spectra of $\mathrm{H} L^{2}$. $\quad$ S9

Figure S3 View of the three unique molecules in the asymmetric unit of $\mathrm{H} L^{1}$. $\mathrm{S} 10$

$\begin{array}{lll}\text { Figure S4 View of the asymmetric unit of } \mathrm{HL}^{2} \text {. } & \mathrm{S} 10\end{array}$

Scheme S1 Potential tautomeric structures of $\mathrm{HL}^{1}$. $\quad \mathrm{S} 11$

Table S4 Hydrogen bond parameters for the crystal structures of $\mathrm{H} L^{1}$ and $\mathrm{H} L^{2}$ S11

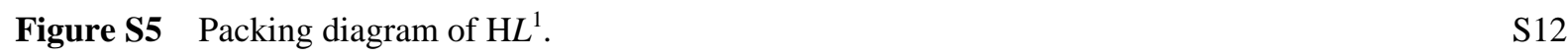

Figure S6 Packing diagram of $\mathrm{H}^{2}$. $\quad \mathrm{S} 13$

Figure S7 The half- $\left[\left\{\mathrm{Fe}\left(\mathrm{OH}_{2}\right)_{6}\right\} \subset \mathrm{Fe}_{8}\left(\mu-L^{1}\right)_{12}\right]^{7+}$ molecule in the asymmetric unit of 1.6MeCN. $\quad \mathrm{S} 14$

Table S5 Selected bond lengths and angles in the crystal structures of 1.6 MeCN. S15

Figure S8 The half- $\left[\left\{\mathrm{Fe}\left(\mathrm{OH}_{2}\right)_{6}\right\} \subset \mathrm{Fe}_{8}\left(\mu-L^{2}\right)_{12}\right]^{7+}$ molecule in the asymmetric unit of $\quad \mathrm{S} 17$ 2. $x \mathrm{MeCN} \cdot y\left(\mathrm{C}_{2} \mathrm{H}_{5}\right)_{2} \mathrm{O}$.

Table S6 Selected bond lengths and angles in the crystal structures of $2 \cdot x \mathrm{MeCN} \cdot y\left(\mathrm{C}_{2} \mathrm{H}_{5}\right)_{2} \mathrm{O} . \quad \mathrm{S} 18$

Table S7 Dihedral angles between the bidentate ligand fragments coordinated to each vertex of the S20 cubane assembly in $\mathbf{1} \cdot 6 \mathrm{MeCN}$.

Table S8 Dihedral angles between the bidentate ligand fragments coordinated to each vertex of the S21 cubane assembly in $\mathbf{2} \cdot x \mathrm{MeCN} \cdot y\left(\mathrm{C}_{2} \mathrm{H}_{5}\right)_{2} \mathrm{O}$.

Table S9 Hydrogen bond parameters for $\mathbf{1} \cdot 6 \mathrm{CH}_{3} \mathrm{CN}$ at $100 \mathrm{~K}$. 22

Table S10 Temperature dependence of the host:guest hydrogen bonds in $1 \cdot 6 \mathrm{MeCN}$ S22

Table S11 Temperature dependence of the host:guest hydrogen bonds in $2 \cdot x \mathrm{MeCN} \cdot y\left(\mathrm{C}_{2} \mathrm{H}_{5}\right)_{2} \mathrm{O} . \quad \mathrm{S} 22$

Table S12 Bond valence sum (BVS) values for the iron atoms in 1.6MeCN.

Table S13 Bond valence sum (BVS) values for the iron atoms in $2 \cdot x \mathrm{MeCN} \cdot y\left(\mathrm{C}_{2} \mathrm{H}_{5}\right)_{2} \mathrm{O} . \quad \mathrm{S} 23$

Figure S9 View of the $\left[\left\{\mathrm{Fe}\left(\mathrm{OH}_{2}\right)_{6}\right\} \subset \mathrm{Fe}_{8}\left(\mu-L^{1}\right)_{12}\right]^{7+}$ assembly, highlighting the intramolecular steric $\quad$ S24 clashes giving rise to the pyrazolyl group disorder in $1 \cdot 6 \mathrm{MeCN}$.

Figure S10 Cut-away space-filling plot of the $\left[\left\{\mathrm{Fe}\left(\mathrm{OH}_{2}\right)_{6}\right\} \subset \mathrm{Fe}_{8}\left(\mu-L^{1}\right)_{12}\right]^{7+}$ assembly. $\quad$ S25

Figure S11 Overlays of the high-spin and low-spin structures of the $\left[\left\{\mathrm{Fe}\left(\mathrm{OH}_{2}\right)_{6}\right\} \subset \mathrm{Fe}_{8}\left(\mu-L^{1}\right)_{12}\right]^{7+} \quad \mathrm{S} 26$ assembly.

Figure S12 Overlays of the high-spin and low-spin structures of the $\left[\left\{\mathrm{Fe}\left(\mathrm{OH}_{2}\right)_{6}\right\} \subset \mathrm{Fe} 8\left(\mu-L^{2}\right)_{12}\right]^{7+} \quad \mathrm{S} 27$ assembly.

$\begin{array}{lll}\text { Figure S13 X-ray powder diffraction data for an air-dried samples of } \mathbf{1} \text { and } \mathbf{2} & \text { S28 }\end{array}$

Figure S14 Magnetic susceptibility data for freshly prepared $\mathbf{1} \cdot 6 \mathrm{CH}_{3} \mathrm{CN}$ and desolvated $\mathbf{1} . \quad$ S29

Figure S15 Magnetic susceptibility data for freshly prepared $2 \cdot x \mathrm{MeCN} \cdot y\left(\mathrm{C}_{2} \mathrm{H}_{5}\right)_{2} \mathrm{O}$ and desolvated $2 . \quad \mathrm{S} 29$

Figure S16 Measured and simulated Mössbauer spectra of $\mathbf{1} . \quad$ S30

Table S14 Parameters from the Mössbauer spectrum simulations. $\quad$ S31

Table S15 Temperature dependence of the high-spin fraction $\left(\gamma_{\mathrm{HS}}\right)$ of the iron(II) centers in $\mathbf{1}$ and $\mathbf{2}, \quad$ S32 as measured by different techniques. 
Figure S17 LIESST effect measurement on 1.

Figure S18 Diffuse reflectance UV/vis spectra of solid 1 at different temperatures. $\quad$ S33

Figure S19 Temperature dependence of the total diffuse reflectance intensity from $1 . \quad S 33$

$\begin{array}{lll}\text { Figure S20 } & { }^{1} \mathrm{H} \text { NMR spectrum of } \mathbf{1}\left(\mathrm{CD}_{3} \mathrm{CN}\right) . & \text { S34 }\end{array}$

$\begin{array}{lll}\text { Figure } \mathbf{S 2 1} & { }^{1} \mathrm{H} \text { NMR spectrum of } \mathbf{2}\left(\mathrm{CD}_{3} \mathrm{CN}\right) . & \mathrm{S} 34\end{array}$

Figure S22 Electrospray mass spectrum of $\mathbf{1}$ from $\mathrm{MeCN}$ solution. $\quad \mathrm{S} 35$

Figure S23 Measured and simulated mass peaks for the ions $\left[\left\{\mathrm{Fe}\left(\mathrm{OH}_{2}\right)_{6}\right\} \subset \mathrm{Fe}_{8}\left(\mu-L^{1}\right)_{12}\left(\mathrm{BF}_{4}\right)_{n}\right]^{(7-n)+} . \quad \mathrm{S} 35$

Figure S24 Electrospray mass spectrum of $\mathbf{2}$ from MeCN solution. $\quad$ S37

Figure S25 Variable temperature magnetic susceptibility data for $\mathbf{1}$ in $\mathrm{CD}_{3} \mathrm{CN} . \quad \mathrm{S} 37$

Figure S26 View of the asymmetric unit in $\left[\mathrm{Fe}\left(\mathrm{HL}^{1}\right)\left(\mathrm{OH}_{2}\right)_{2}(\mathrm{NCMe})_{2}\right]\left[\mathrm{ClO}_{4}\right]_{2}(\mathbf{3}) . \quad \mathrm{S} 38$

Figure S27 View of the asymmetric unit in $\left[\mathrm{Fe}\left(\mathrm{HL}^{1}\right)\left(\mathrm{OH}_{2}\right)_{3}(\mathrm{NCMe})\right]\left[\mathrm{BF}_{4}\right]_{2}(\mathbf{4}) . \quad \mathrm{S} 39$

Table S16 Selected bond lengths and angles in the crystal structure of $\mathbf{3}$ S40

Table S17 Hydrogen bond parameters for the crystal structures of $3 . \quad S 40$

Table S18 Selected bond lengths and angles in the crystal structure of $4 . \quad$ S41

Table S19 Hydrogen bond parameters for the crystal structures of $4 . \quad$ S41

Figure S28 Packing diagram of 4, showing the 2D assembly of the hydrogen-bonded dimers. $\quad$ S42

Figure S29 View of the asymmetric unit in catena- $\left[\mathrm{Fe}\left(L^{2}\right)\left(\mathrm{OCMe}_{2}\right)\left(\mathrm{OH}_{2}\right)\right]\left[\mathrm{BF}_{4}\right]_{2} \cdot \mathrm{H}_{2} \mathrm{O}\left(\mathbf{5} \cdot \mathrm{H}_{2} \mathrm{O}\right) . \quad \mathrm{S} 43$

Table S20 Selected bond lengths and angles in the crystal structure of $\mathbf{5} \cdot \mathrm{H}_{2} \mathrm{O} . \quad \mathrm{S} 43$

Table S21 Hydrogen bond parameters for the crystal structure of $\mathbf{5} \cdot \mathrm{H}_{2} \mathrm{O}$. S44

Figure S30 Packing diagram of $\mathbf{5} \cdot \mathrm{H}_{2} \mathrm{O}$, showing the coordination polymer chains. $\quad \mathrm{S} 44$

Figure S31 Measured and simulated X-ray powder diffraction data for $\mathbf{3}$ and $\mathbf{5} \cdot \mathrm{H}_{2} \mathrm{O}$. S45

$\begin{array}{ll}\text { References } & \text { S46 }\end{array}$ 


\section{Experimental}

\section{Single Crystal Structure Analyses}

Experimental details of the structure determinations in this study are given in Tables S1-S3. Unless otherwise stated, all crystallographically ordered non- $\mathrm{H}$ atoms in each structure were refined anisotropically, and $\mathrm{H}$ atoms were placed in calculated positions and refined using a riding model.

Structure refinements of $\mathbf{H} \boldsymbol{L}^{1}$ and $\mathbf{H} \boldsymbol{L}^{2}$. The asymmetric unit of $\mathrm{H} L^{1}$ contains three molecules of the compound, while $\mathrm{H} L^{2}$ has jsut one molecule in its asymmetric unit. No disorder is present in either model. All $\mathrm{H}$ atoms were located in the Fourier map and refined, with $U_{\text {iso }}$ constrained to $1.2 \mathrm{x} U_{\text {eq }}$ of the corresponding $\mathrm{C}$ or $\mathrm{N}$ atom for the heterocyclic $\mathrm{H}$ atoms, or $1.5 \mathrm{x} U_{\mathrm{eq}}\{\mathrm{C}\}$ for the $\mathrm{H} L^{2}$ methyl groups.

Structure refinements of $\left[\left\{\mathrm{Fe}\left(\mathrm{OH}_{2}\right)_{6}\right\} \subset \mathrm{Fe}_{8}\left(\mu-L^{\mathbf{1}}\right)_{12}\right]\left[\mathrm{BF}_{4}\right]_{7} \cdot \mathbf{6 C H}_{3} \mathrm{CN}(1 \cdot \mathrm{MeCN})$. The asymmetric unit contains half a formula unit, with the encapsulated $\mathrm{Fe}$ ion $\mathrm{Fe}(5)$ lying on the inversion center $3 / 4,1 / 4,1 / 2$. Five pyrazolyl rings on the six unique ligands are disordered over two equally occupied sites, which is related to close inter-ligand steric contacts across faces of the cubic assembly (Figure S9). While distance restraints were used to construct the ligand disorder model, these were removed for the final cycles of least squares refinement. There are four unique $\mathrm{BF}_{4}^{-}$ions, one of which lies close to the $C_{2}$ axis $1 / 2, y, 3 / 4$ and so is only half-occupied. This half-anion is crystallographically ordered at 100 and $150 \mathrm{~K}$, but disordered over two sites at higher temperatures. The other three whole anions are disordered over two or three orientations which were modelled with refined B-F and F...F restraints; isotropic displacement ellipsoid restraints were applied to some disordered $\mathrm{F}$ atoms in the higher temperature refinements. There are four fully or part-occupied acetonitrile sites in the model, three of which are disordered. The fixed restraints $\mathrm{C}-\mathrm{C}=1.48(2), \mathrm{C}-\mathrm{N}=$ $1.15(2)$ and 1,3-C...N = 2.63(2) $\AA$ were applied to those molecules.

At $100 \mathrm{~K}$ the aqua ligand $\mathrm{H}$ atoms successfully refined, with the restraints $\mathrm{O}-\mathrm{H}=0.90(1)$ and $\mathrm{H} . . . \mathrm{H}=$ $1.56(1) \AA$ and with $U_{\text {iso }}$ constrained to $1.5 \mathrm{x} U_{\text {eq }}\{\mathrm{O}\}$. At higher temperatures these $\mathrm{H}$ atoms positions did not refine satisfactorily, so they were omitted from the model. The highest residual Fourier peak of $1.0-1.5 e \AA^{-3}$ in the lower temperature refinements lies on the special position near the half-occupied $\mathrm{BF}_{4}^{-}$ion site.

Structure refinements of $\left[\left\{\mathrm{Fe}\left(\mathrm{OH}_{2}\right)_{6}\right\} \subset \mathrm{Fe}_{8}\left(\mu-L^{2}\right)_{12}\right]\left[\mathrm{BF}_{4}\right]_{7} \cdot x \mathrm{CH}_{3} \mathrm{CN} \cdot y\left(\mathrm{C}_{2} \mathrm{H}_{5}\right)_{2} \mathrm{O}\left(2 \cdot x \mathrm{MeCN} \cdot y\left(\mathrm{C}_{2} \mathrm{H}_{5}\right)_{2} \mathrm{O}\right)$. The asymmetric unit contains half a formula unit, with the encapsulated Fe ion Fe(5) lying on the inversion center $1 / 2,1 / 2,1 / 2$. There are four unique $\mathrm{BF}_{4}{ }^{-}$ions, three of which are crystallographically ordered at 125 and $200 \mathrm{~K}$ but disordered at $250 \mathrm{~K}$. The fourth anion is disordered at all temperatures over three equally populated sites whose occupancies sum to 0.5 . The disordered anions were treated with fixed B-F and F...F distance restraints and, in some cases, with isotropic displacement ellipsoid restraints. There are three or four part-occupied acetonitrile sites in the refinements, which were refined with the restraints $\mathrm{C}-\mathrm{C}=1.48(2)$, $\mathrm{C}-\mathrm{N}=1.15(2)$ and 1,3-C...N $=2.63(2) \AA$; and, two or three partial diethyl ether molecules, which were treated with the restraints $\mathrm{C}-\mathrm{C}=1.52(2), \mathrm{C}-\mathrm{O}=1.43(2), 1,3-\mathrm{C} \ldots \mathrm{C}=2.34(2)$ and $1,3-\mathrm{C} \ldots \mathrm{O}=2.42(2) \AA$.

All fully occupied non-H atoms plus (at $125 \mathrm{~K}$ ) selected partial solvent sites, were refined anisotropically. The aqua ligand $\mathrm{H}$ atoms positions were not evident in the Fourier map and so were not included in the model. The highest residual Fourier peak of 1.3-1.6 $e \AA^{-3}$ lies within a $\mathrm{BF}_{4}^{-}$ion site, or (at 125 $\mathrm{K})$ in a partial diethyl ether molecule.

The $125 \mathrm{~K}$ refinement includes 2.5 equiv of acetonitrile and diethyl ether (ie $x=y=2.5$ ), but the 200 and $250 \mathrm{~K}$ structures contain reduced amounts of each solvent. Evidently the solvent was slowly lost during data collection at these higher temperatures. Consistent with that, attempts to collect a fourth dataset from the same crystal led to its complete decomposition.

Structure refinement of $\left[\mathrm{Fe}\left(\mathrm{HL}^{1}\right)_{2}\left(\mathrm{OH}_{2}\right)_{2}(\mathrm{NCMe})_{2}\right]\left[\mathrm{ClO}_{4}\right]_{2}(3)$ Two non-superimposable twin domains were resolved in the $h k l$ file, with a population ratio of 0.80:0.20. The asymmetric unit contains two formula units of the compound, with all residues in general crystallographic positions. One of the four unique perchlorate ions is disordered over two sites, with refined occupancies of 0.61 and 0.39 . These were modelled using the refined restraints $\mathrm{Cl}-\mathrm{O}=1.43(2)$ and $\mathrm{O} \ldots \mathrm{O}=2.34(2) \AA$. All non- $\mathrm{H}$ atoms except the minor anion disorder site were refined anisotropically. The water ligand $\mathrm{H}$ atoms were located in the Fourier map and refined positionally, with the fixed restraints $\mathrm{O}-\mathrm{H}=0.90(2)$ and $\mathrm{H} . . . \mathrm{H}=1.47(2) \AA$ and with $U_{\text {iso }}(\mathrm{H})$ $=1.5 \times U_{\text {eq }}(\mathrm{O})$. The highest residual Fourier peak of $+1.3 e \AA^{-3}$ is associated with an ordered anion, and may indicate a minor degree of unmodelled disorder in that residue. 


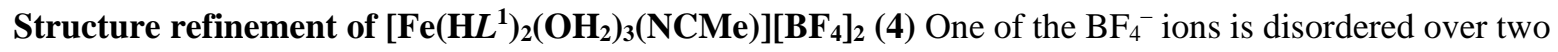
sites, which were refined as equally occupied. These were modelled using the refined restraints $\mathrm{B}-\mathrm{F}=$ $1.39(2)$ and $\mathrm{F} . . . \mathrm{F}=2.27(2) \AA$. All fully occupied non-H atoms were refined anisotropically and C-bound $\mathrm{H}$ atoms were placed in calculated positions and refined using a riding model. The $\mathrm{N}-\mathrm{H}$ and water ligand $\mathrm{H}$ atoms were located in the Fourier map and refined positionally, with the fixed restraint $\mathrm{O}-\mathrm{H}=0.90(2) \AA$ applied to the latter. The thermal parameter constraints $U_{\text {iso }}(\mathrm{H})=1.2 \times U_{\text {eq }}(\mathrm{N})$ or $1.5 \times U_{\text {eq }}(\mathrm{O})$ were also applied to these $\mathrm{H}$ atoms.

Structure refinement of $\left[\mathrm{Fe}\left(\mu-L^{3}\right)\left(\mathrm{OH}_{2}\right)\left(\mathrm{OCMe}_{2}\right)\right]\left[\mathrm{BF}_{4}\right]_{2} \cdot \mathrm{H}_{2} \mathrm{O}\left(5 \cdot \mathrm{H}_{2} \mathrm{O}\right)$ The asymmetric unit of this crystal contains one formula unit of the compound, with all residues on general crystallographic sites. One $\mathrm{BF}_{4}^{-}$ion is disordered over two orientations, whose occupancies refined to 0.71:0.29. This was modelled with refined restraints $\mathrm{B}-\mathrm{F}=1.38(2)$ and $\mathrm{F} . . \mathrm{F}=2.25(2) \AA$. All non-H atoms except the minor anion disorder site were refined anisotropically. The water $\mathrm{H}$ atoms were located in the Fourier map and refined, with fixed $\mathrm{O}-\mathrm{H}=$ $0.90(2) \AA$ and $U_{\text {iso }}(\mathrm{H})=1.5 \mathrm{x} U_{\mathrm{eq}}(\mathrm{O})$.

CCDC-1861171-1861177 and 1938349-1938353 contain the supplementary crystallographic data for this paper (Table S1). These data can be obtained free of charge from The Cambridge Crystallographic Data Center via www.ccdc.cam.ac.uk/data_request/cif. 
Table S1 Experimental data for the crystal structure determinations of $1 \cdot 6 \mathrm{CH}_{3} \mathrm{CN}$.

\begin{tabular}{|c|c|c|c|c|}
\hline$T / \mathrm{K}$ & 100 & 150 & 200 & 250 \\
\hline molecular formula & & $\mathrm{C}_{132} \mathrm{H}$ & & \\
\hline$M_{\mathrm{r}}$ & & & & \\
\hline crystal class & & & & \\
\hline space group & & & & \\
\hline$a / \AA$ & $28.138(12)$ & $28.1814(3)$ & $28.3179(3)$ & $28.4475(3)$ \\
\hline$b / \AA$ & $20.942(11)$ & $20.9721(2)$ & $21.0820(2)$ & $21.1969(2)$ \\
\hline$c / \AA$ & $29.140(17)$ & $29.1619(4)$ & $29.2509(3)$ & $29.3353(4)$ \\
\hline$\alpha /^{\circ}$ & - & - & - & - \\
\hline$\beta /^{\circ}$ & $93.17(4)$ & $93.064(1)$ & $92.810(1)$ & $92.571(1)$ \\
\hline$\gamma /{ }^{\circ}$ & - & - & - & - \\
\hline$V / \AA^{3}$ & $17145(15)$ & $17210.7(3)$ & $17441.7(3)$ & $17671.3(3)$ \\
\hline$Z$ & 4 & 4 & 4 & 4 \\
\hline$\mu / \mathrm{mm}^{-1}$ & $0.854^{\mathrm{a}}$ & $0.851^{\mathrm{a}}$ & $0.839^{\mathrm{a}}$ & $0.766^{\mathrm{a}}$ \\
\hline$D_{\mathrm{c}} / \mathrm{gcm}^{-3}$ & 1.624 & 1.618 & 1.596 & 1.575 \\
\hline measured reflections & 125210 & 35961 & 36429 & 36842 \\
\hline independent reflections & 21605 & 12633 & 12794 & 12946 \\
\hline$R_{\text {int }}$ & 0.062 & 0.037 & 0.032 & 0.034 \\
\hline parameters & 1236 & 1237 & 1271 & 1271 \\
\hline restraints & 108 & 108 & 192 & 194 \\
\hline$R_{1}\left[F_{0}>4 \sigma\left(F_{0}\right)\right]^{\mathrm{b}}$ & 0.081 & 0.079 & 0.076 & 0.080 \\
\hline$w R_{2}$, all data ${ }^{c}$ & 0.274 & 0.260 & 0.255 & 0.264 \\
\hline goodness of fit & 1.102 & 1.132 & 1.124 & 1.099 \\
\hline$\Delta \rho_{\min / \max } / e \AA^{-3}$ & $+1.49 /-0.84$ & $+1.16 /-0.74$ & $+0.93 /-0.62$ & $+0.77 /-0.53$ \\
\hline $\mathrm{CCDC}$ & 1861172 & 1861173 & 1861174 & 1861175 \\
\hline
\end{tabular}

${ }^{\mathrm{a}}$ Collected with synchrotron radiation. $\quad{ }^{\mathrm{b}} R=\Sigma\left[\left|F_{\mathrm{o}}\right|-\left|F_{\mathrm{c}}\right|\right] / \Sigma\left|F_{\mathrm{o}}\right| \quad{ }^{\mathrm{c}} w R=\left[\Sigma w\left(F_{\mathrm{o}}{ }^{2}-F_{\mathrm{c}}{ }^{2}\right) / \Sigma w F_{\mathrm{o}}^{4}\right]^{1 / 2}$ 
Table S2 Experimental data for the crystal structure determinations of $2 \cdot x \mathrm{MeCN} \cdot y \mathrm{Et}_{2} \mathrm{O}$. The solvent content of the crystal, given by $x$ and $y$, appears to decrease at higher temperatures which may indicate partial solvent loss during the higher temperature data collections.

\begin{tabular}{|c|c|c|c|}
\hline$T / \mathrm{K}$ & $\begin{array}{c}125 \\
(x=2.5, y=2.5)\end{array}$ & $\begin{array}{c}200 \\
(x=2.5, y=1.5)\end{array}$ & $\begin{array}{c}250 \\
(x=1.8, y=1.3)\end{array}$ \\
\hline molecular formula & $\mathrm{C}_{159} \mathrm{H}_{176.5} \mathrm{~B}_{7} \mathrm{~F}_{28} \mathrm{Fe}_{9} \mathrm{~N}_{74.5} \mathrm{O}_{20.5}$ & $\mathrm{C}_{155} \mathrm{H}_{166.5} \mathrm{~B}_{7} \mathrm{~F}_{28} \mathrm{Fe}_{9} \mathrm{~N}_{74.5} \mathrm{O}_{19.5}$ & $\mathrm{C}_{152.8} \mathrm{H}_{162.4} \mathrm{~B}_{7} \mathrm{~F}_{28} \mathrm{Fe}_{9} \mathrm{~N}_{73.8} \mathrm{O}_{19.3}$ \\
\hline$M_{\mathrm{r}}$ & 4569.57 & 4495.45 & 4451.89 \\
\hline crystal class & & monoclinic & \\
\hline space group & & $P 2_{1} / n$ & \\
\hline$a / \AA$ & $17.2790(4)$ & $17.4408(2)$ & $17.4969(5)$ \\
\hline$b / \AA$ & $24.2218(5)$ & $24.2929(2)$ & $24.4003(11)$ \\
\hline$c / \AA$ & $25.7224(4)$ & $25.9866(3)$ & $25.9749(5)$ \\
\hline$\alpha /^{\circ}$ & - & - & - \\
\hline$\beta / \circ$ & $99.479(2)$ & $99.074(1)$ & $98.569(2)$ \\
\hline$\gamma /{ }^{\circ}$ & - & - & - \\
\hline$V / \AA^{3}$ & $10618.6(4)$ & $10872.4(2)$ & $10965.7(6)$ \\
\hline Z & 2 & 2 & 2 \\
\hline$\mu / \mathrm{mm}^{-1}$ & $5.646^{\mathrm{a}}$ & $5.502^{\mathrm{a}}$ & $5.448^{\mathrm{a}}$ \\
\hline$D_{\mathrm{c}} / \mathrm{gcm}^{-3}$ & 1.429 & 1.373 & 1.348 \\
\hline measured reflections & 47892 & 44615 & 45815 \\
\hline independent reflections & 20478 & 21326 & 21426 \\
\hline$R_{\text {int }}$ & 0.041 & 0.024 & 0.033 \\
\hline parameters & 1417 & 1395 & 1380 \\
\hline restraints & 69 & 68 & 131 \\
\hline$R_{1}\left[F_{0}>4 \sigma\left(F_{0}\right)\right]^{\mathrm{b}}$ & 0.067 & 0.057 & 0.071 \\
\hline$w R_{2}$, all data ${ }^{\mathrm{c}}$ & 0.196 & 0.178 & 0.223 \\
\hline goodness of fit & 1.028 & 1.027 & 1.030 \\
\hline$\Delta \rho_{\min / \max } / e \AA^{-3}$ & $+1.57 /-1.00$ & $+1.28 /-0.62$ & $+1.30 /-0.95$ \\
\hline CCDC & 1938349 & 1938353 & 1938352 \\
\hline
\end{tabular}

${ }^{\mathrm{a} C o l l e c t e d}$ with $\mathrm{Cu}-K_{\alpha}$ radiation.

${ }^{\mathrm{b}} R=\Sigma\left[\left|F_{\mathrm{o}}\right|-\left|F_{\mathrm{c}}\right|\right] / \Sigma\left|F_{\mathrm{o}}\right|$

${ }^{\mathrm{c}} w R=\left[\Sigma w\left(F_{\mathrm{o}}{ }^{2}-F_{\mathrm{c}}{ }^{2}\right) / \Sigma w F_{\mathrm{o}}{ }^{4}\right]^{1 / 2}$ 
Table S3 Experimental data for the other crystal structure determinations.

\begin{tabular}{|c|c|c|c|c|c|}
\hline$T / \mathrm{K}$ & $\begin{array}{c}H \mathrm{~L}^{1} \\
100 \\
\end{array}$ & $\begin{array}{l}H \mathrm{~L}^{2} \\
125 \\
\end{array}$ & $\begin{array}{c}3 \\
120 \\
\end{array}$ & $\begin{array}{c}4 \\
125 \\
\end{array}$ & $\begin{array}{c}\mathbf{5} \cdot \mathrm{H}_{2} \mathrm{O} \\
120 \\
\end{array}$ \\
\hline molecular formula & $\mathrm{C}_{10} \mathrm{H}_{8} \mathrm{~N}_{6} \mathrm{O}$ & $\mathrm{C}_{12} \mathrm{H}_{12} \mathrm{~N}_{6} \mathrm{O}$ & $\mathrm{C}_{14} \mathrm{H}_{18} \mathrm{Cl}_{2} \mathrm{FeN}_{8} \mathrm{O}_{11}$ & $\mathrm{C}_{12} \mathrm{H}_{17} \mathrm{~B}_{2} \mathrm{~F}_{8} \mathrm{FeN} \mathrm{N}_{7} \mathrm{O}_{4}$ & $\mathrm{C}_{14} \mathrm{H}_{20} \mathrm{~B}_{2} \mathrm{~F}_{8} \mathrm{FeN}_{6} \mathrm{O}_{3}$ \\
\hline$M_{\mathrm{r}}$ & 228.22 & 256.28 & 601.11 & 552.80 & 549.83 \\
\hline crystal class & monoclinic & triclinic & triclinic & triclinic & monoclinic \\
\hline space group & $P 2_{1} / c$ & $P \overline{1}$ & $P \overline{1}$ & $P \overline{1}$ & $P 2_{1} / n$ \\
\hline$a / \AA$ & $3.7553(1)$ & $6.3701(4)$ & $10.4570(3)$ & $10.0049(9)$ & $11.1084(2)$ \\
\hline$b / \AA$ & $17.3319(4)$ & $8.0327(6)$ & $15.2881(4)$ & $10.1934(11)$ & $9.5675(1)$ \\
\hline$c / \AA$ & $44.326(1)$ & $12.0083(11)$ & $15.3002(4)$ & 11.1633(11) & $21.0007(4)$ \\
\hline$\alpha /^{\circ}$ & - & 77.757(7) & $86.767(2)$ & $109.358(9)$ & - \\
\hline$\beta /^{\circ}$ & $92.170(3)$ & $88.305(6)$ & $77.965(3)$ & $94.272(7)$ & $102.628(2)$ \\
\hline$\gamma / \circ$ & - & $75.102(6)$ & 76.781(2) & $91.322(8)$ & - \\
\hline$V / \AA^{3}$ & $2882.95(14)$ & $580.09(8)$ & $2328.76(11)$ & $1069.75(18)$ & $2177.96(6)$ \\
\hline Z & 12 & 2 & 4 & 2 & 4 \\
\hline$\mu / \mathrm{mm}^{-1}$ & $0.112^{\mathrm{a}}$ & $0.837^{\mathrm{b}}$ & $7.983^{b}$ & $6.653^{\mathrm{b}}$ & $6.481^{\mathrm{b}}$ \\
\hline$D_{\mathrm{c}} / \mathrm{gcm}^{-3}$ & 1.577 & 1.467 & 1.715 & 1.716 & 1.677 \\
\hline measured reflections & 35410 & 4021 & 15126 & 8475 & 8404 \\
\hline independent reflections & 8769 & 2144 & $15126^{\mathrm{c}}$ & 4018 & 4261 \\
\hline$R_{\text {int }}$ & 0.186 & 0.036 & $0^{c}$ & 0.033 & 0.021 \\
\hline parameters & 532 & 208 & 699 & 325 & 344 \\
\hline restraints & 0 & 0 & 32 & 26 & 24 \\
\hline$R_{1}\left[F_{0}>4 \sigma\left(F_{0}\right)\right]^{\mathrm{d}}$ & 0.078 & 0.051 & 0.058 & 0.053 & 0.037 \\
\hline$w R_{2}$, all data $\mathrm{e}^{\mathrm{e}}$ & 0.189 & 0.142 & 0.169 & 0.140 & 0.097 \\
\hline goodness of fit & 0.915 & 1.037 & 1.087 & 1.020 & 1.052 \\
\hline$\Delta \rho_{\min / \max } / e \AA^{-3}$ & $-0.41 / 0.49$ & $-0.29 / 0.28$ & $+1.25 /-0.82$ & $+0.99 /-0.60$ & $+0.47 /-0.35$ \\
\hline CCDC & 1861171 & 1938350 & 1861176 & 1938351 & 1861177 \\
\hline
\end{tabular}

${ }^{\mathrm{a}}$ Collected with synchrotron radiation. $\quad{ }^{\mathrm{b}}$ Collected with $\mathrm{Cu}-K_{\alpha}$ radiation.

${ }^{\mathrm{d}} R=\Sigma\left[\left|F_{\mathrm{o}}\right|-\left|F_{\mathrm{c}}\right|\right] / \Sigma\left|F_{\mathrm{o}}\right| \quad{ }^{\mathrm{e}} w R=\left[\Sigma w\left(F_{\mathrm{o}}{ }^{2}-F_{\mathrm{c}}{ }^{2}\right) / \Sigma w F_{\mathrm{o}}{ }^{4}\right]^{1 / 2}$ 


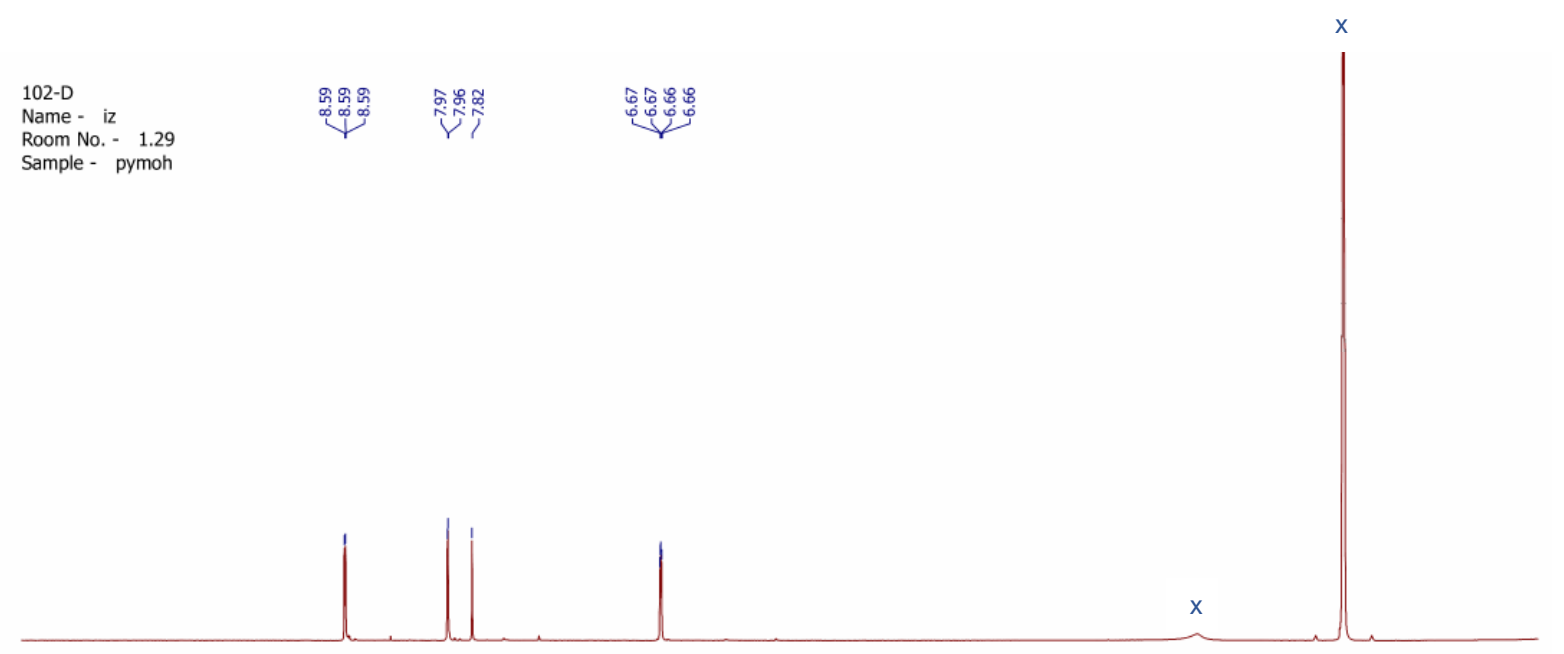

\begin{tabular}{|c|c|c|c|c|c|c|c|c|c|c|c|c|c|c|c|c|c|c|}
\hline 10 & 100 & 9.5 & 90 & 8.5 & 80 & 75 & 70 & 6.5 & 6.0 & 5.5 & 5.0 & 4.5 & 4.0 & 3.5 & 3.0 & 2.5 & 2.0 & 1.5 \\
\hline 10.5 & 10.0 & 9.5 & 9.0 & 8.5 & 8.0 & 7.5 & 7.0 & 6.5 & $\begin{array}{l}6.0 \\
\mathrm{f} 1(\mathrm{ppm})\end{array}$ & 5.5 & 5.0 & 4.5 & 4.0 & 3.5 & 3.0 & 2.5 & 2.0 & 1.5 \\
\hline
\end{tabular}
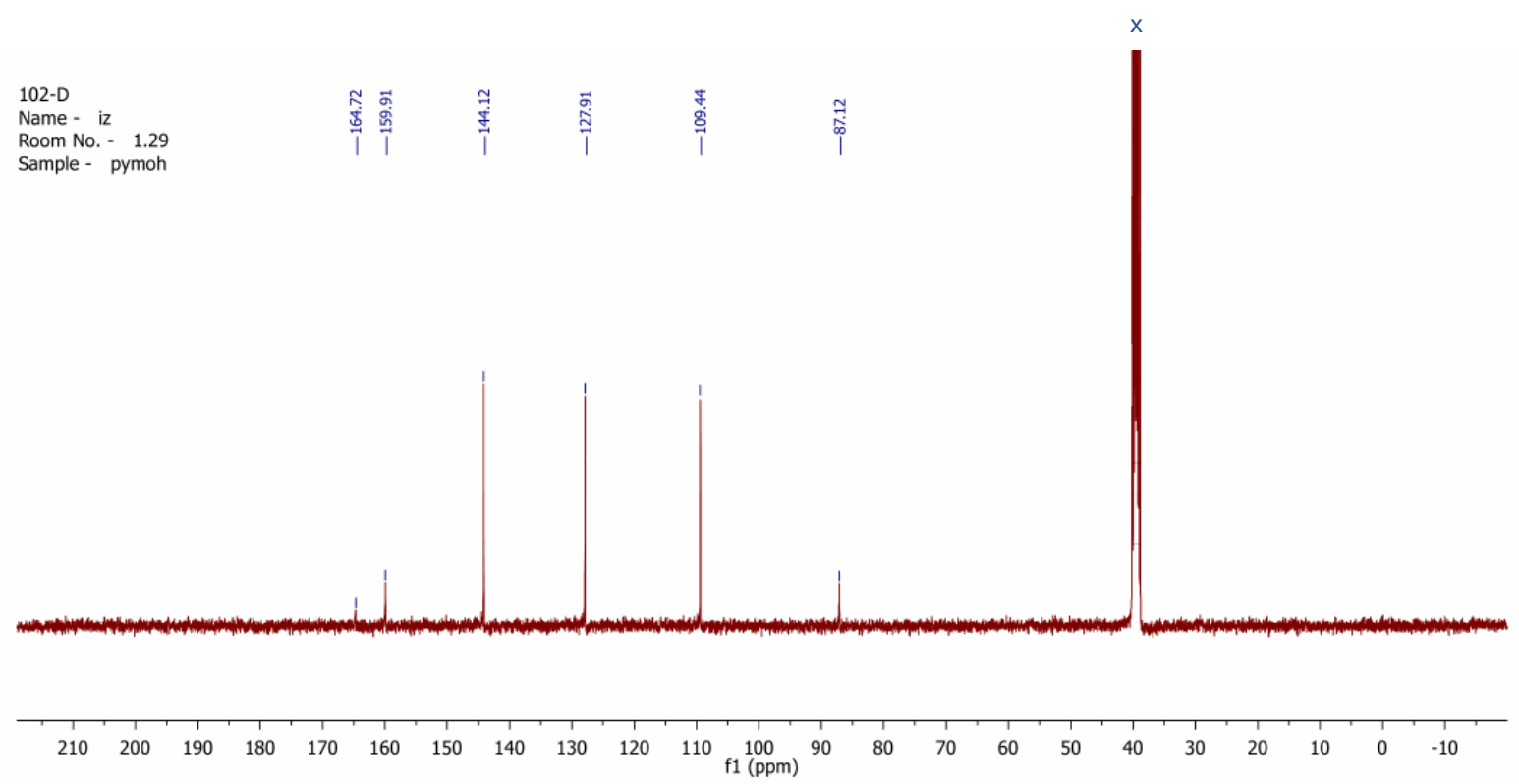

Figure $\mathbf{S 1}{ }^{1} \mathrm{H}$ (top) and ${ }^{13} \mathrm{C}$ (bottom) NMR spectra of $\mathrm{H} L^{1}\left(\left\{\mathrm{CD}_{3}\right\}_{2} \mathrm{SO}\right)$.

No $\mathrm{N} H$ peak was observed in the ${ }^{1} \mathrm{H}$ spectrum of $\mathrm{H} L^{1}$, which probably reflects chemical exchange of these protons with adventitious water. Consistent with that, the water peak at $3.3 \mathrm{ppm}$ in this spectrum is very broad. The $\mathrm{N} H$ proton in $\mathrm{HL}^{2}$ was observed by ${ }^{1} \mathrm{H}$ NMR, at $12.5 \mathrm{ppm}$ (Figure $\mathrm{S} 2$ ). 
$161-4 \quad 161-4 \stackrel{\Re}{n}$
Name -
Room No. -1.25
Sample - $161-4$

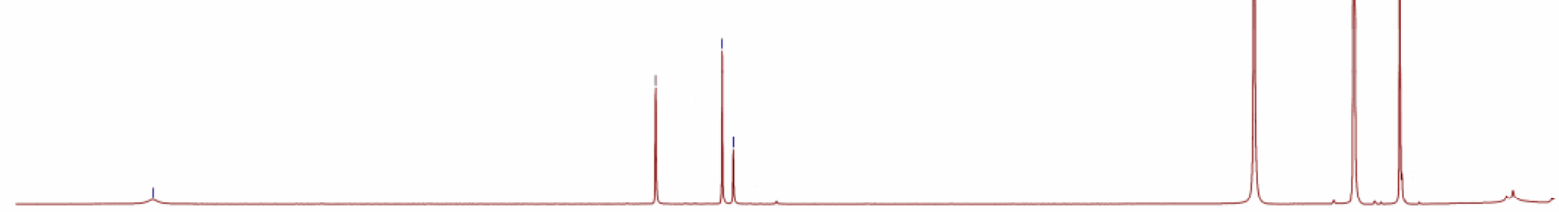
$\begin{array}{rrrrrrrrrrrrrrrrrrrrrrrrrrrrr} & 13.5 & 13.0 & 12.5 & 12.0 & 11.5 & 11.0 & 10.5 & 10.0 & 9.5 & 9.0 & 8.5 & 8.0 & 7.5 & 7.0 & 6.5 & 6.0 & 5.5 & 5.0 & 4.5 & 4.0 & 3.5 & 3.0 & 2.5 & 2.0 & 1.5 & 1.0\end{array}$

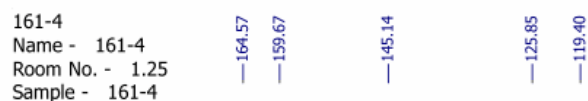

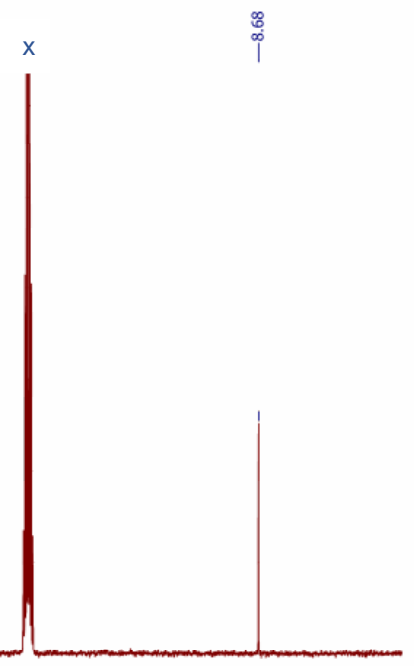

\begin{tabular}{|c|c|c|c|c|c|c|c|c|c|c|c|c|c|c|c|c|c|c|c|c|}
\hline $\begin{array}{c}1 \\
190\end{array}$ & $\begin{array}{c}1 \\
180\end{array}$ & $\begin{array}{l}1 \\
170\end{array}$ & $\begin{array}{c}1 \\
160\end{array}$ & $\begin{array}{l}1 \\
150\end{array}$ & 140 & $\begin{array}{c}1 \\
130\end{array}$ & 12 & 110 & & $\begin{array}{l}1 \\
90\end{array}$ & 80 & 70 & 10 & $\begin{array}{l}1 \\
50\end{array}$ & $\begin{array}{l}1 \\
40\end{array}$ & 30 & $\begin{array}{l}1 \\
20\end{array}$ & $\begin{array}{l}1 \\
10\end{array}$ & T & -10 \\
\hline 190 & 180 & 170 & 160 & 150 & 140 & 130 & 120 & 110 & & ppm) & 80 & 70 & 60 & 30 & 40 & 30 & 20 & 10 & 0 & -10 \\
\hline
\end{tabular}

Figure S2 ${ }^{1} \mathrm{H}$ (top) and ${ }^{13} \mathrm{C}$ (bottom) NMR spectra of $\mathrm{HL}{ }^{2}\left(\left\{\mathrm{CD}_{3}\right\}_{2} \mathrm{SO}\right)$. 


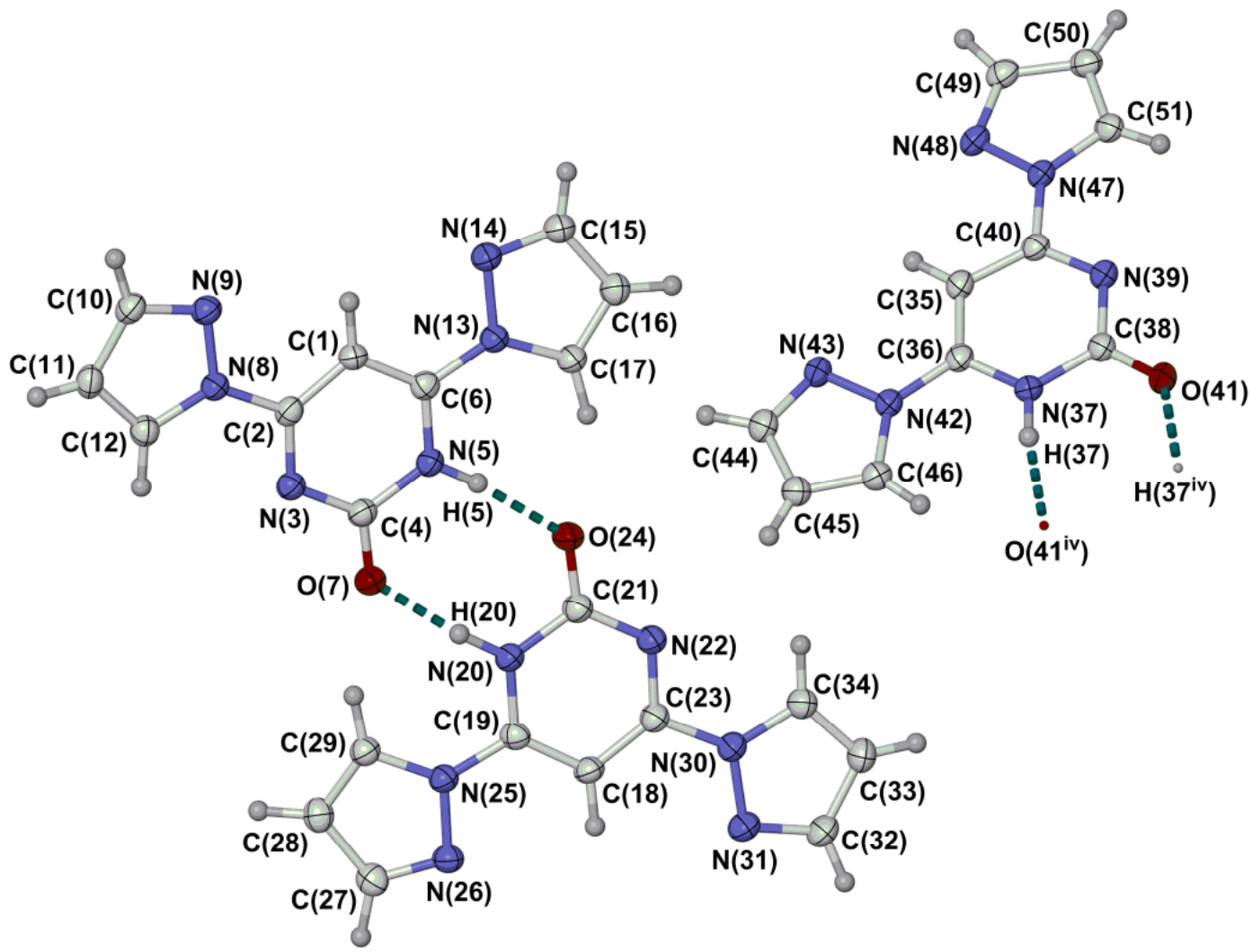

Figure S3 View of the three unique molecules in the asymmetric unit of $\mathrm{H} L^{1}$. Displacement ellipsoids are at the $50 \%$ probability level, except for $\mathrm{H}$ atoms which have arbitrary radii. Symmetry code: (iv) $2-x, 1-y,-z$. Color code $\mathrm{C}$, white; $\mathrm{H}$, grey; $\mathrm{N}$, blue; $\mathrm{O}$, red.

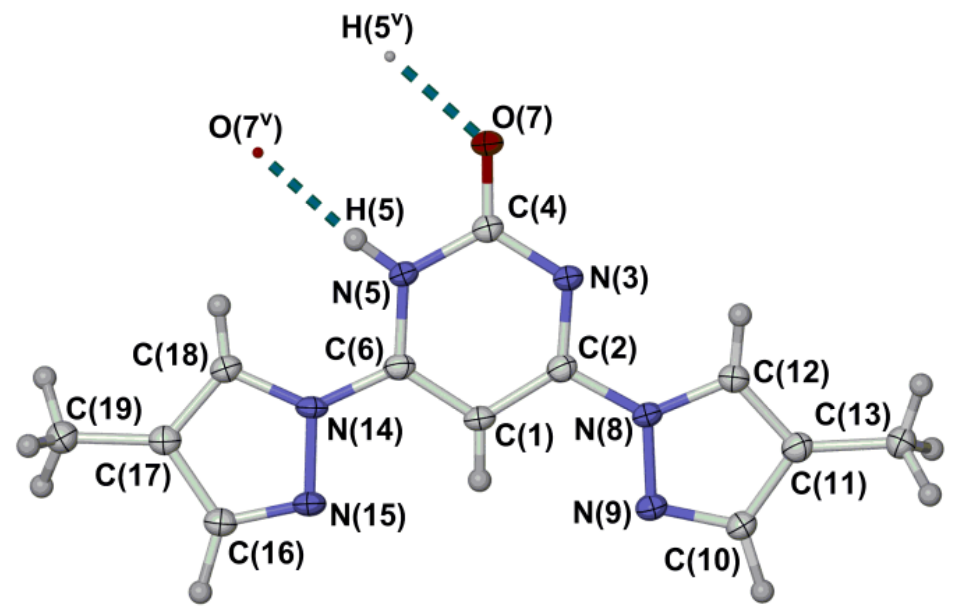

Figure S4 View of the asymmetric unit of $\mathrm{H} L^{2}$. Details as for Figure S3. Symmetry code: (v) $-x, 1-y, 1-z$. 

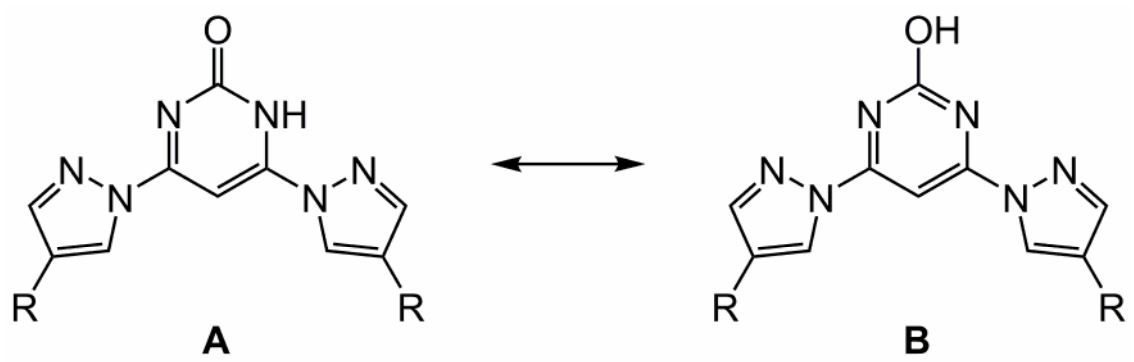

Scheme S1 Potential tautomeric structures of $\mathrm{H} L^{1}$ and $\mathrm{H} L^{2}(\mathrm{R}=\mathrm{H}$ or Me). Tautomer $\mathrm{A}$ is adopted by the free ligands in the solid state and in solution, but the $\left[L^{1}\right]^{-}$and $\left[L^{2}\right]^{-}$ligands in $\mathbf{1}$ and $\mathbf{2}$ are better described as a deprotonated form of tautomer B.

The presence of the 4,6-di(pyrazol-1-yl)-1H-pyrimid-2-one tautomer in the structures of $\mathrm{H} L^{1}$ and $\mathrm{H} L^{2}$, rather than the alternative 2-hydroxy-4,6-di(pyrazol-1-yl)-1H-pyrimidine form (Scheme S1), is evident for two reasons. First is the location and refinement of the N-bound $\mathrm{H}$ atoms in the Fourier map. Second, is from the $\mathrm{C}=\mathrm{O}$ distances in each molecule which lie between 1.240(2)-1.249(3) $\AA$ and clearly indicate a double bond between these atoms. The ${ }^{13} \mathrm{C}$ NMR chemical shift for the pyrimidinyl $C 2$ atom $\left(\delta 164.7\right.$ for $\mathrm{H} L^{1}$ and 164.6 $\left.\mathrm{H} L^{2}\right)$ implies the same tautomer is also present in $\left(\mathrm{CD}_{3}\right)_{2} \mathrm{SO}$ solution (Figures $\mathrm{S} 1$ and $\left.\mathrm{S} 2\right)^{1}$

Table S4 Hydrogen bond parameters for the crystal structures of $\mathrm{H} L^{1}$ and $\mathrm{H} L^{2}\left(\AA,{ }^{\circ}\right)$. See Figures S3 and S4 for the atom numbering schemes. Symmetry codes: (iv) $2-x, 1-y,-z$; (v) $-x, 1-y, 1-z$.

\begin{tabular}{lcccc}
\hline & $\mathrm{D}-\mathrm{H}$ & $\mathrm{H} \ldots \mathrm{A}$ & $\mathrm{D} \ldots \mathrm{A}$ & $\mathrm{D}-\mathrm{H} \ldots \mathrm{A}$ \\
\hline $\mathrm{H} L^{1}$ & & & & \\
$\mathrm{~N}(5)-\mathrm{H}(5) \ldots \mathrm{O}(24)$ & $0.90(3)$ & $1.87(3)$ & $2.765(2)$ & $172(2)$ \\
$\mathrm{N}(20)-\mathrm{H}(20) \ldots \mathrm{O}(7)$ & $0.94(2)$ & $1.83(3)$ & $2.760(2)$ & $170(2)$ \\
$\mathrm{N}(37)-\mathrm{H}(37) \ldots \mathrm{O}\left(41^{\mathrm{iv}}\right)$ & $0.88(3)$ & $1.89(3)$ & $2.748(2)$ & $165(3)$ \\
& & & & \\
$\mathrm{H} L^{2}$ & & & & \\
$\mathrm{~N}(5)-\mathrm{H}(5) \ldots \mathrm{O}\left(7^{\vee}\right)$ & $0.86(3)$ & $1.88(3)$ & $2.728(2)$ & $174(2)$ \\
\hline
\end{tabular}




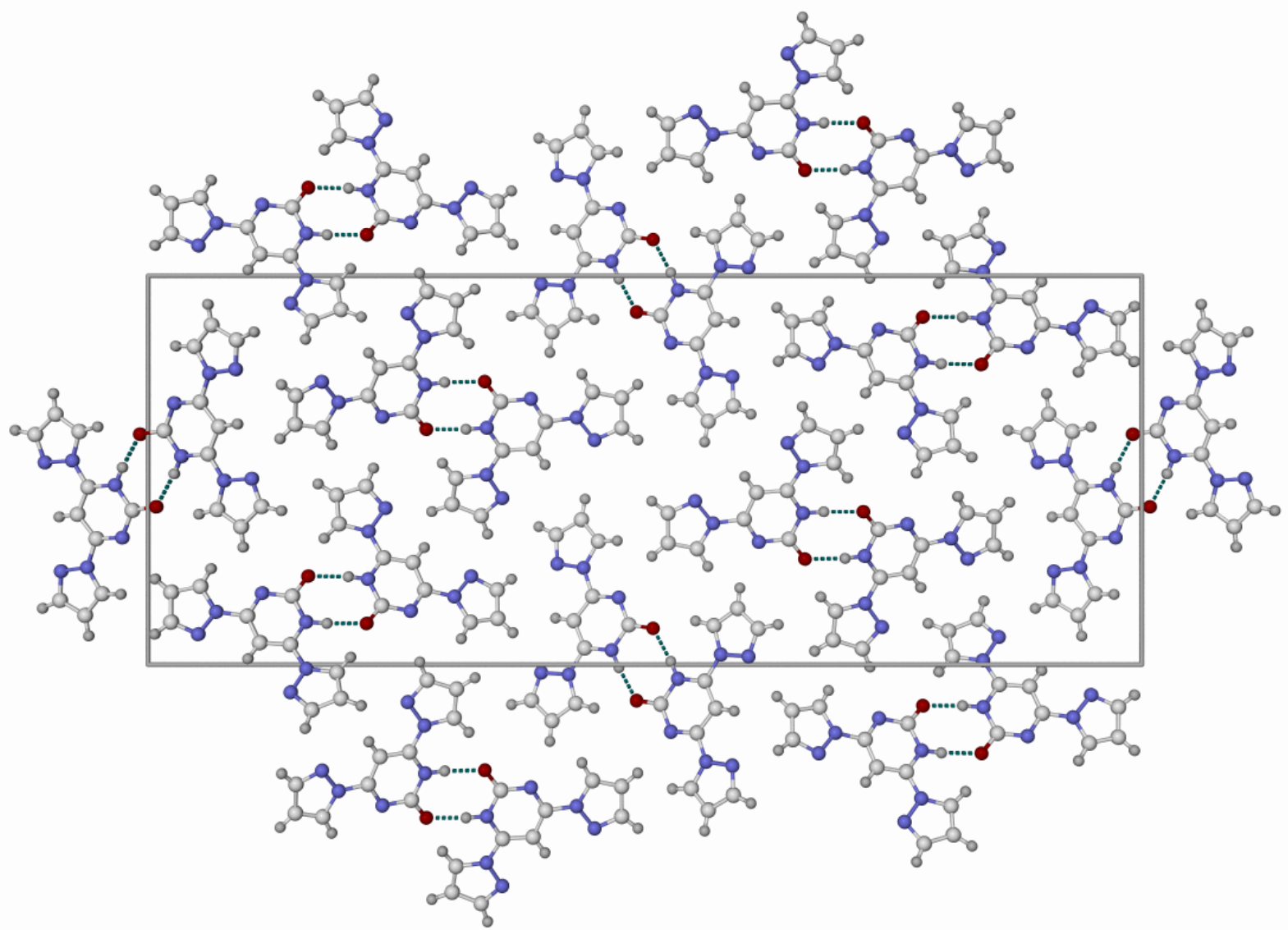

Figure S5 Packing diagram of $H \mathrm{~L}^{1}$. The view is parallel to the [100] crystal vector, with the unit cell $b$ axis horizontal.

Hydrogen-bonded molecular dyads are arranged in canted stacks by translation along the $a$ direction. The interplanar distance between adjacent molecules in the stacks is 3.248(6)-3.335(3) A. 


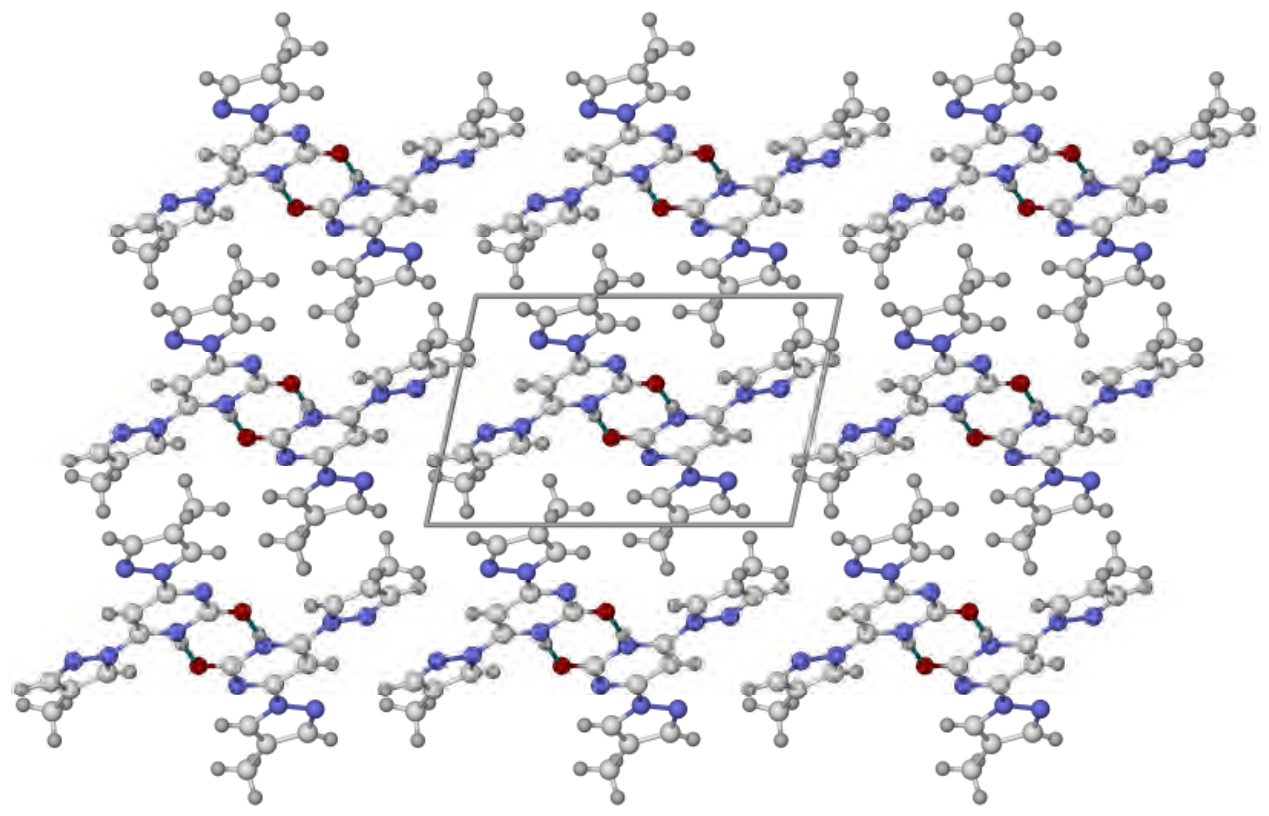

Figure S6 Packing diagram of $H \mathrm{~L}^{2}$. The view is parallel to the [100] crystal vector, with the unit cell $c$ axis horizontal.

Hydrogen-bonded molecular dyads are arranged in canted stacks by translation along the $a$ direction. The interplanar distance between adjacent molecules in the stacks is 3.270(4) $\AA$. 


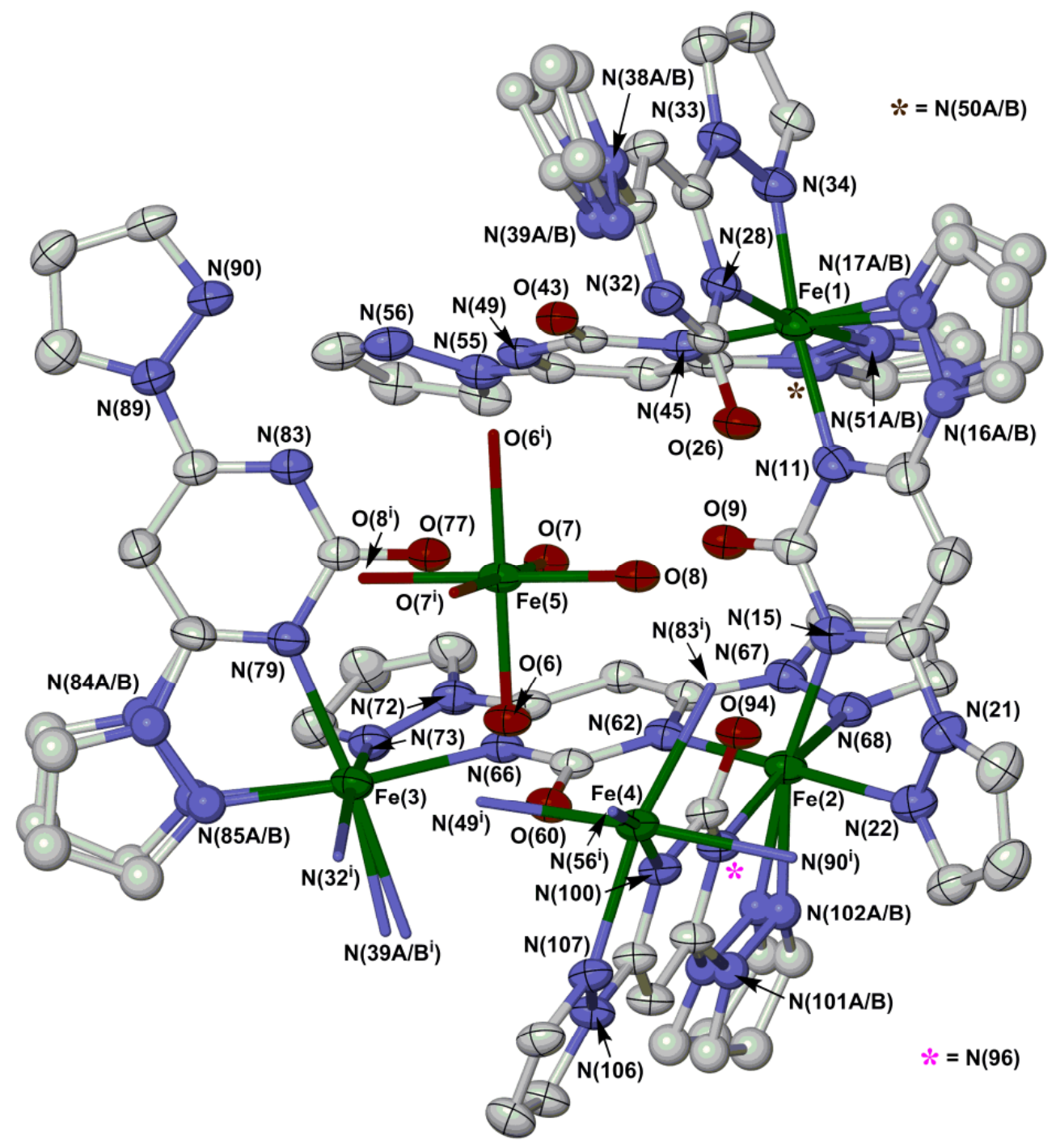

Figure S7 View of the half- $\left[\left\{\mathrm{Fe}\left(\mathrm{OH}_{2}\right)_{6}\right\} \subset \mathrm{Fe}_{8}\left(\mu-L^{1}\right)_{12}\right]^{7+}$ assembly in the asymmetric unit of $\mathbf{1} \cdot 6 \mathrm{CH}_{3} \mathrm{CN}$ at $100 \mathrm{~K}$, showing the numbering of the metal and ligand heteroatoms. Both orientations of the disordered pyrazolyl groups in the model are shown. Displacement ellipsoids are at the $50 \%$ probability level, and $\mathrm{H}$ atoms are omitted for clarity. Symmetry code: (i) $3 / 2-x, 1 / 2-y, 1-z$.

Color code: $\mathrm{C}$, white; $\mathrm{Fe}$, green; $\mathrm{N}$, blue; $\mathrm{O}$, red. 
Table S5 Selected bond lengths and angles in the crystal structures of $1 \cdot 6 \mathrm{MeCN}\left(\AA,{ }^{\circ}\right)$. See Fig. S7 for the atom numbering scheme employed. Symmetry code: (i) $3 / 2-x,{ }^{1} / 2-y, 1-z$.

\begin{tabular}{|c|c|c|c|c|}
\hline$T / \mathrm{K}$ & 100 & 150 & 200 & 250 \\
\hline $\mathrm{Fe}(1)-\mathrm{N}(11)$ & $2.066(3)$ & $2.079(5)$ & $2.120(5)$ & $2.149(4)$ \\
\hline $\mathrm{Fe}(1)-\mathrm{N}(17 \mathrm{~A} / \mathrm{B})$ & $1.854(7) / 2.184(8)$ & $1.829(12) / 2.211(12)$ & $1.884(11) / 2.297(11)$ & $1.971(12) / 2.343(13)$ \\
\hline $\mathrm{Fe}(1)-\mathrm{N}(28)$ & $2.055(3)$ & $2.078(4)$ & $2.109(4)$ & $2.145(4)$ \\
\hline $\mathrm{Fe}(1)-\mathrm{N}(34)$ & $1.989(3)$ & $2.017(4)$ & $2.076(4)$ & $2.141(4)$ \\
\hline $\mathrm{Fe}(1)-\mathrm{N}(45)$ & $2.053(3)$ & $2.068(5)$ & $2.107(4)$ & $2.145(4)$ \\
\hline $\mathrm{Fe}(1)-\mathrm{N}(51 \mathrm{~A} / \mathrm{B})$ & $2.034(7) / 1.951(8)$ & $2.031(14) / 1.995(13)$ & $2.108(14) / 2.041(11)$ & $2.158(18) / 2.100(15)$ \\
\hline $\mathrm{Fe}(2)-\mathrm{N}(15)$ & $2.156(3)$ & $2.158(4)$ & $2.154(4)$ & $2.152(4)$ \\
\hline $\mathrm{Fe}(2)-\mathrm{N}(22)$ & $2.159(4)$ & $2.189(5)$ & $2.199(5)$ & $2.203(5)$ \\
\hline $\mathrm{Fe}(2)-\mathrm{N}(62)$ & 2.171(3) & $2.182(4)$ & $2.194(4)$ & $2.203(5)$ \\
\hline $\mathrm{Fe}(2)-\mathrm{N}(68)$ & $2.152(3)$ & $2.164(5)$ & $2.184(4)$ & $2.188(5)$ \\
\hline $\mathrm{Fe}(2)-\mathrm{N}(96)$ & $2.166(3)$ & $2.180(4)$ & $2.189(4)$ & 2.184(4) \\
\hline $\mathrm{Fe}(2)-\mathrm{N}(102 \mathrm{~A} / \mathrm{B})$ & $2.128(8) / 2.140(7)$ & $2.126(11) / 2.180(10)$ & $2.158(10) / 2.182(9)$ & $2.138(11) / 2.188(9)$ \\
\hline $\mathrm{Fe}(3)-\mathrm{N}\left(32^{\mathrm{i}}\right)$ & $2.179(3)$ & $2.184(5)$ & $2.176(4)$ & $2.164(4)$ \\
\hline $\mathrm{Fe}(3)-\mathrm{N}\left(39 \mathrm{~A} / \mathrm{B}^{\mathrm{i}}\right)$ & $2.242(9) / 2.160(8)$ & $2.236(12) / 2.169(12)$ & $2.243(10) / 2.173(10)$ & $2.248(10) / 2.158(10)$ \\
\hline $\mathrm{Fe}(3)-\mathrm{N}(66)$ & $2.168(3)$ & $2.168(4)$ & $2.171(4)$ & $2.168(4)$ \\
\hline $\mathrm{Fe}(3)-\mathrm{N}(73)$ & $2.225(3)$ & $2.231(5)$ & $2.231(4)$ & $2.229(5)$ \\
\hline $\mathrm{Fe}(3)-\mathrm{N}(79)$ & $2.201(3)$ & $2.207(5)$ & $2.198(4)$ & $2.204(4)$ \\
\hline $\mathrm{Fe}(3)-\mathrm{N}(85 \mathrm{~A} / \mathrm{B})$ & $2.041(8) / 2.264(8)$ & $2.072(11) / 2.274(12)$ & $2.041(11) / 2.264(10)$ & $1.974(16) / 2.252(11)$ \\
\hline $\mathrm{Fe}(4)-\mathrm{N}\left(49^{\mathrm{i}}\right)$ & $2.200(3)$ & $2.192(4)$ & $2.191(4)$ & $2.188(4)$ \\
\hline $\mathrm{Fe}(4)-\mathrm{N}\left(56^{\mathrm{i}}\right)$ & $2.192(3)$ & $2.193(4)$ & $2.196(4)$ & $2.198(5)$ \\
\hline $\mathrm{Fe}(4)-\mathrm{N}\left(83^{\mathrm{i}}\right)$ & $2.179(3)$ & $2.177(4)$ & $2.184(4)$ & $2.196(4)$ \\
\hline $\mathrm{Fe}(4)-\mathrm{N}\left(90^{\mathrm{i}}\right)$ & $2.192(4)$ & $2.189(5)$ & $2.187(4)$ & $2.180(5)$ \\
\hline $\mathrm{Fe}(4)-\mathrm{N}(100)$ & 2.192(3) & $2.182(4)$ & $2.181(4)$ & $2.183(4)$ \\
\hline $\mathrm{Fe}(4)-\mathrm{N}(107)$ & $2.182(3)$ & $2.187(4)$ & $2.194(4)$ & $2.205(4)$ \\
\hline $\mathrm{Fe}(5)-\mathrm{O}(6)$ & $1.993(2)$ & $2.002(3)$ & $1.995(3)$ & $1.993(3)$ \\
\hline $\mathrm{Fe}(5)-\mathrm{O}(7)$ & $2.005(2)$ & $2.007(4)$ & 2.013(3) & $2.019(3)$ \\
\hline $\mathrm{Fe}(5)-\mathrm{O}(8)$ & $1.996(3)$ & $2.008(4)$ & $2.006(4)$ & $2.004(4)$ \\
\hline $\mathrm{Fe}(1) \ldots \mathrm{Fe}(2)$ & $6.216(3)$ & $6.2139(10)$ & $6.2256(9)$ & $6.2371(9)$ \\
\hline $\mathrm{Fe}(1) \ldots \mathrm{Fe}\left(3^{i}\right)$ & $6.241(3)$ & $6.2334(11)$ & $6.2417(10)$ & $6.2417(10)$ \\
\hline $\mathrm{Fe}(1) \ldots \mathrm{Fe}\left(4^{\mathrm{i}}\right)$ & $6.252(4)$ & $6.2515(12)$ & $6.2587(11)$ & $6.2667(11)$ \\
\hline $\mathrm{Fe}(2) \ldots \mathrm{Fe}(3)$ & $6.279(4)$ & $6.2827(12)$ & $6.2891(11)$ & $6.2935(12)$ \\
\hline $\mathrm{Fe}(2) \ldots \mathrm{Fe}(4)$ & $6.327(3)$ & $6.3137(10)$ & $6.3232(9)$ & $6.3284(10)$ \\
\hline $\mathrm{Fe}(3) \ldots \mathrm{Fe}\left(4^{i}\right)$ & $6.351(3)$ & $6.3450(10)$ & $6.3560(9)$ & $6.3625(9)$ \\
\hline $\mathrm{Fe}(1) \ldots \mathrm{Fe}\left(1^{\mathrm{i}}\right)$ & $11.321(6)$ & $11.2949(16)$ & $11.2431(14)$ & $11.1668(15)$ \\
\hline $\mathrm{Fe}(2) \ldots \mathrm{Fe}\left(2^{i}\right)$ & $10.675(4)$ & $10.6723(16)$ & $10.7199(15)$ & $10.7714(15)$ \\
\hline $\mathrm{Fe}(3) \ldots \mathrm{Fe}\left(3^{\mathrm{i}}\right)$ & $10.932(4)$ & $10.9450(16)$ & $10.9909(15)$ & $11.0386(15)$ \\
\hline $\mathrm{Fe}(4) \ldots \mathrm{Fe}\left(4^{\mathrm{i}}\right)$ & $10.548(4)$ & $10.5346(15)$ & $10.5583(14)$ & $10.5794(15)$ \\
\hline $\mathrm{N}(11)-\mathrm{Fe}(1)-\mathrm{N}(17 \mathrm{~A} / \mathrm{B})$ & $82.4(2) / 74.5(2)$ & $82.3(4) / 73.7(4)$ & $80.4(3) / 72.0(3)$ & $77.4(4) / 71.0(3)$ \\
\hline $\mathrm{N}(11)-\mathrm{Fe}(1)-\mathrm{N}(28)$ & $102.40(13)$ & 103.09(17) & $104.80(15)$ & 106.51(16) \\
\hline $\mathrm{N}(11)-\mathrm{Fe}(1)-\mathrm{N}(34)$ & $173.19(14)$ & $172.54(19)$ & $171.03(18)$ & $169.86(19)$ \\
\hline $\mathrm{N}(11)-\mathrm{Fe}(1)-\mathrm{N}(45)$ & 100.62(12) & 101.26(17) & 102.50(16) & 103.76(16) \\
\hline $\mathrm{N}(11)-\mathrm{Fe}(1)-\mathrm{N}(51 \mathrm{~A} / \mathrm{B})$ & $90.0(2) / 83.2(3)$ & $90.3(4) / 84.4(4)$ & $90.8(4) / 84.3(3)$ & $90.4(4) / 85.3(4)$ \\
\hline $\mathrm{N}(17 \mathrm{~A} / \mathrm{B})-\mathrm{Fe}(1)-\mathrm{N}(28)$ & $84.9(2) / 81.6(2)$ & $84.0(3) / 82.5(3)$ & $83.4(3) / 83.4(3)$ & $82.7(3) / 83.5(3)$ \\
\hline $\mathrm{N}(17 \mathrm{~A} / \mathrm{B})-\mathrm{Fe}(1)-\mathrm{N}(34)$ & $91.2(2) / 99.4(2)$ & 90.5(4)/99.3(4) & $90.9(3) / 99.5(3)$ & $92.8(4) / 99.3(3)$ \\
\hline $\mathrm{N}(17 \mathrm{~A} / \mathrm{B})-\mathrm{Fe}(1)-\mathrm{N}(45)$ & $172.5(2) / 174.9(2)$ & $171.9(4) / 173.9(3)$ & $171.8(3) / 172.5(3)$ & $172.2(3) / 171.9(3)$ \\
\hline $\mathrm{N}(17 \mathrm{~A} / \mathrm{B})-\mathrm{Fe}(1)-\mathrm{N}(51 \mathrm{~A} / \mathrm{B})$ & $89.9(3)-103.6(3)$ & $91.0(5)-102.9(5)$ & $92.2(4)-102.6(5)$ & $93.3(5)-101.7(6)$ \\
\hline $\mathrm{N}(28)-\mathrm{Fe}(1)-\mathrm{N}(34)$ & $79.27(13)$ & 78.19(18) & $76.18(16)$ & $74.58(16)$ \\
\hline $\mathrm{N}(28)-\mathrm{Fe}(1)-\mathrm{N}(45)$ & 101.01(12) & $102.16(18)$ & 103.09(16) & 104.09(16) \\
\hline $\mathrm{N}(28)-\mathrm{Fe}(1)-\mathrm{N}(51 \mathrm{~A} / \mathrm{B})$ & $167.5(2) / 171.7(2)$ & $166.5(4) / 170.3(4)$ & $164.4(4) / 169.0(3)$ & $163.1(4) / 166.2(4)$ \\
\hline $\mathrm{N}(34)-\mathrm{Fe}(1)-\mathrm{N}(45)$ & 85.44(13) & $85.53(18)$ & $85.81(16)$ & $85.51(17)$ \\
\hline $\mathrm{N}(34)-\mathrm{Fe}(1)-\mathrm{N}(51 \mathrm{~A} / \mathrm{B})$ & $88.7(2) / 94.5(3)$ & $88.7(4) / 93.6(4)$ & $88.5(4) / 93.8(3)$ & $88.6(4) / 92.5(4)$ \\
\hline $\mathrm{N}(45)-\mathrm{Fe}(1)-\mathrm{N}(51 \mathrm{~A} / \mathrm{B})$ & $74.7(2) / 83.8(3)$ & $73.3(4) / 82.2(4)$ & $72.1(4) / 80.5(3)$ & 71.7(5)/79.2(4) \\
\hline $\mathrm{N}(15)-\mathrm{Fe}(2)-\mathrm{N}(22)$ & 74.57(13) & 74.21(18) & 74.24(17) & 74.12(17) \\
\hline $\mathrm{N}(15)-\mathrm{Fe}(2)-\mathrm{N}(62)$ & $108.18(11)$ & $108.88(16)$ & $109.25(16)$ & $109.60(16)$ \\
\hline $\mathrm{N}(15)-\mathrm{Fe}(2)-\mathrm{N}(68)$ & 84.72(11) & 84.91(16) & $85.05(15)$ & $85.33(15)$ \\
\hline $\mathrm{N}(15)-\mathrm{Fe}(2)-\mathrm{N}(96)$ & $104.25(11)$ & $104.59(15)$ & 104.51(14) & $103.84(15)$ \\
\hline $\mathrm{N}(15)-\mathrm{Fe}(2)-\mathrm{N}(102 \mathrm{~A} / \mathrm{B})$ & $162.5(3) / 171.5(2)$ & $161.0(4) / 171.0(3)$ & $159.8(3) / 170.8(3)$ & $158.5(4) / 170.4(3)$ \\
\hline
\end{tabular}


Table S5 continued.

\begin{tabular}{|c|c|c|c|c|}
\hline$T[\mathrm{~K}]$ & 100 & 150 & 200 & 250 \\
\hline $\mathrm{N}(22)-\mathrm{Fe}(2)-\mathrm{N}(62)$ & $173.56(12)$ & $173.10(17)$ & $172.60(15)$ & $171.75(16)$ \\
\hline $\mathrm{N}(22)-\mathrm{Fe}(2)-\mathrm{N}(68)$ & $100.02(13)$ & $99.77(19)$ & $99.99(17)$ & $98.98(19)$ \\
\hline $\mathrm{N}(22)-\mathrm{Fe}(2)-\mathrm{N}(96)$ & $81.30(13)$ & 81.31(18) & $80.96(16)$ & $81.39(17)$ \\
\hline $\mathrm{N}(22)-\mathrm{Fe}(2)-\mathrm{N}(102 \mathrm{~A} / \mathrm{B})$ & $88.5(3) / 97.0(2)$ & $87.3(4) / 96.8(3)$ & $85.9(3) / 96.5(3)$ & $84.6(3) / 96.3(3)$ \\
\hline $\mathrm{N}(62)-\mathrm{Fe}(2)-\mathrm{N}(68)$ & $74.69(12)$ & $74.66(17)$ & $74.15(16)$ & $74.33(18)$ \\
\hline $\mathrm{N}(62)-\mathrm{Fe}(2)-\mathrm{N}(96)$ & 103.33(12) & 103.49(17) & 104.03(15) & 104.39(16) \\
\hline $\mathrm{N}(62)-\mathrm{Fe}(2)-\mathrm{N}(102 \mathrm{~A} / \mathrm{B})$ & $88.3(2) / 80.2(2)$ & $89.1(4) / 80.1(3)$ & $90.1(3) / 79.9(3)$ & $91.2(3) / 80.0(3)$ \\
\hline $\mathrm{N}(68)-\mathrm{Fe}(2)-\mathrm{N}(96)$ & 170.91(11) & $170.30(15)$ & $170.23(14)$ & $170.48(15)$ \\
\hline $\mathrm{N}(68)-\mathrm{Fe}(2)-\mathrm{N}(102 \mathrm{~A} / \mathrm{B})$ & $94.4(2) / 96.4(2)$ & $94.2(3) / 97.3(3)$ & $95.1(3) / 96.6(3)$ & $95.7(3) / 97.0(3)$ \\
\hline $\mathrm{N}(96)-\mathrm{Fe}(2)-\mathrm{N}(102 \mathrm{~A} / \mathrm{B})$ & $76.6(2) / 74.5(2)$ & $76.2(3) / 73.0(3)$ & $75.2(3) / 73.7(3)$ & $74.8(3) / 73.5(3)$ \\
\hline $\mathrm{N}\left(32^{\mathrm{i}}\right)-\mathrm{Fe}(3)-\mathrm{N}\left(39 \mathrm{~A} / \mathrm{B}^{\mathrm{i}}\right)$ & $72.8(2) / 75.2(2)$ & $72.8(3) / 75.3(3)$ & $72.3(3) / 75.2(3)$ & $73.1(3) / 74.1(3)$ \\
\hline $\mathrm{N}\left(32^{\mathrm{i}}\right)-\mathrm{Fe}(3)-\mathrm{N}(66)$ & $107.96(12)$ & $108.12(17)$ & $108.06(16)$ & $107.86(16)$ \\
\hline $\mathrm{N}\left(32^{\mathrm{i}}\right)-\mathrm{Fe}(3)-\mathrm{N}(73)$ & $166.70(12)$ & $166.26(17)$ & $166.00(15)$ & $165.68(16)$ \\
\hline $\mathrm{N}\left(32^{\mathrm{i}}\right)-\mathrm{Fe}(3)-\mathrm{N}(79)$ & 108.97(12) & 108.87(17) & 109.11(15) & $109.21(15)$ \\
\hline $\mathrm{N}\left(32^{\mathrm{i}}\right)-\mathrm{Fe}(3)-\mathrm{N}(85 \mathrm{~A} / \mathrm{B})$ & $83.8(3) / 94.0(2)$ & $83.8(4) / 94.5(4)$ & $83.3(4) / 93.8(3)$ & $83.9(5) / 92.6(3)$ \\
\hline $\mathrm{N}\left(39 \mathrm{~A} / \mathrm{B}^{\mathrm{i}}\right)-\mathrm{Fe}(3)-\mathrm{N}(66)$ & $82.8(2) / 89.2(2)$ & $82.3(3) / 89.9(4)$ & $82.0(3) / 90.5(3)$ & $81.2(3) / 91.5(3)$ \\
\hline $\mathrm{N}\left(39 \mathrm{~A} / \mathrm{B}^{\mathrm{i}}\right)-\mathrm{Fe}(3)-\mathrm{N}(73)$ & $94.3(2) / 91.6(2)$ & $93.9(3) / 91.1(3)$ & $94.4(3) / 90.9(3)$ & 93.3(3)/91.7(3) \\
\hline $\mathrm{N}\left(39 \mathrm{~A} / \mathrm{B}^{\mathrm{i}}\right)-\mathrm{Fe}(3)-\mathrm{N}(79)$ & $175.1(3) / 167.2(3)$ & $175.7(4) / 166.3(4)$ & $175.9(3) / 165.4(3)$ & $175.6(3) / 164.7(3)$ \\
\hline $\mathrm{N}\left(39 \mathrm{~A} / \mathrm{B}^{\mathrm{i}}\right)-\mathrm{Fe}(3)-\mathrm{N}(85 \mathrm{~A} / \mathrm{B})$ & $93.7(3)-101.9(3)$ & $93.0(4)-102.4(4)$ & $92.3(4)-102.6(4)$ & $90.7(5)-103.2(4)$ \\
\hline $\mathrm{N}(66)-\mathrm{Fe}(3)-\mathrm{N}(73)$ & $72.87(12)$ & $72.73(17)$ & $73.31(16)$ & $73.60(17)$ \\
\hline $\mathrm{N}(66)-\mathrm{Fe}(3)-\mathrm{N}(79)$ & $100.66(11)$ & $100.79(16)$ & $101.00(14)$ & 101.37(15) \\
\hline $\mathrm{N}(66)-\mathrm{Fe}(3)-\mathrm{N}(85 \mathrm{~A} / \mathrm{B})$ & $168.2(3) / 157.9(2)$ & $168.2(4) / 157.3(3)$ & $168.6(4) / 157.9(3)$ & $168.2(5) / 159.4(3)$ \\
\hline $\mathrm{N}(73)-\mathrm{Fe}(3)-\mathrm{N}(79)$ & $83.58(12)$ & $84.16(18)$ & $83.88(16)$ & $84.05(16)$ \\
\hline $\mathrm{N}(73)-\mathrm{Fe}(3)-\mathrm{N}(85 \mathrm{~A} / \mathrm{B})$ & $95.6(3) / 85.2(2)$ & $95.7(4) / 84.8(4)$ & $95.6(4) / 84.8(3)$ & $94.7(5) / 86.0(3)$ \\
\hline $\mathrm{N}(79)-\mathrm{Fe}(3)-\mathrm{N}(85 \mathrm{~A} / \mathrm{B})$ & $75.1(2) / 73.6(2)$ & $74.8(3) / 73.6(3)$ & $74.7(3) / 73.6(3)$ & $75.0(5) / 73.1(3)$ \\
\hline $\mathrm{N}\left(49^{\mathrm{i}}\right)-\mathrm{Fe}(4)-\mathrm{N}\left(56^{\mathrm{i}}\right)$ & 73.64(12) & $73.59(17)$ & $73.73(16)$ & 73.74(17) \\
\hline $\mathrm{N}\left(49^{\mathrm{i}}\right)-\mathrm{Fe}(4)-\mathrm{N}\left(83^{\mathrm{i}}\right)$ & $108.17(11)$ & $108.21(16)$ & $107.97(14)$ & $107.80(15)$ \\
\hline $\mathrm{N}\left(49^{\mathrm{i}}\right)-\mathrm{Fe}(4)-\mathrm{N}\left(90^{\mathrm{i}}\right)$ & $166.93(11)$ & $166.45(16)$ & $166.01(15)$ & $165.49(16)$ \\
\hline $\mathrm{N}\left(49^{\mathrm{i}}\right)-\mathrm{Fe}(4)-\mathrm{N}(100)$ & $107.15(11)$ & $107.20(16)$ & $107.11(15)$ & 107.71(16) \\
\hline $\mathrm{N}\left(49^{\mathrm{i}}\right)-\mathrm{Fe}(4)-\mathrm{N}(107)$ & $81.85(11)$ & $81.67(16)$ & $81.95(15)$ & $81.78(16)$ \\
\hline $\mathrm{N}\left(56^{\mathrm{i}}\right)-\mathrm{Fe}(4)-\mathrm{N}\left(83^{\mathrm{i}}\right)$ & $83.44(12)$ & $83.58(17)$ & $83.68(15)$ & $83.39(16)$ \\
\hline $\mathrm{N}\left(56^{\mathrm{i}}\right)-\mathrm{Fe}(4)-\mathrm{N}\left(90^{\mathrm{i}}\right)$ & $93.88(13)$ & $93.49(18)$ & $92.76(17)$ & 92.21(19) \\
\hline $\mathrm{N}\left(56^{\mathrm{i}}\right)-\mathrm{Fe}(4)-\mathrm{N}(100)$ & $168.81(11)$ & $168.15(16)$ & $167.57(15)$ & $167.32(16)$ \\
\hline $\mathrm{N}\left(56^{\mathrm{i}}\right)-\mathrm{Fe}(4)-\mathrm{N}(107)$ & $95.40(12)$ & $95.23(17)$ & $94.59(16)$ & $94.18(17)$ \\
\hline $\mathrm{N}\left(83^{\mathrm{i}}\right)-\mathrm{Fe}(4)-\mathrm{N}\left(90^{\mathrm{i}}\right)$ & $73.41(12)$ & $73.55(17)$ & $73.31(15)$ & $73.33(17)$ \\
\hline $\mathrm{N}\left(83^{\mathrm{i}}\right)-\mathrm{Fe}(4)-\mathrm{N}(100)$ & 106.51(11) & $106.95(16)$ & $107.39(14)$ & $107.61(15)$ \\
\hline $\mathrm{N}\left(83^{\mathrm{i}}\right)-\mathrm{Fe}(4)-\mathrm{N}(107)$ & 168.99(12) & $169.12(18)$ & $168.85(17)$ & $168.85(18)$ \\
\hline $\mathrm{N}\left(90^{\mathrm{i}}\right)-\mathrm{Fe}(4)-\mathrm{N}(100)$ & $84.33(12)$ & $84.59(17)$ & $85.28(15)$ & $85.19(17)$ \\
\hline $\mathrm{N}\left(90^{\mathrm{i}}\right)-\mathrm{Fe}(4)-\mathrm{N}(107)$ & $95.80(12)$ & $95.78(17)$ & $95.82(16)$ & $95.95(17)$ \\
\hline $\mathrm{N}(100)-\mathrm{Fe}(4)-\mathrm{N}(107)$ & $73.85(11)$ & $73.39(16)$ & $73.45(15)$ & $73.81(15)$ \\
\hline $\mathrm{O}(6)-\mathrm{Fe}(5)-\mathrm{O}\left(6^{\mathrm{i}}\right)$ & 180 & 180 & 180 & 180 \\
\hline $\mathrm{O}(6)-\mathrm{Fe}(5)-\mathrm{O}(7)$ & $89.64(10)$ & $89.38(14)$ & $89.61(13)$ & $89.71(14)$ \\
\hline $\mathrm{O}(6)-\mathrm{Fe}(5)-\mathrm{O}\left(7^{\mathrm{i}}\right)$ & $90.36(10)$ & $90.62(14)$ & $90.39(13)$ & $90.29(14)$ \\
\hline $\mathrm{O}(6)-\mathrm{Fe}(5)-\mathrm{O}(8)$ & $89.71(10)$ & $89.72(15)$ & $89.75(14)$ & $90.18(14)$ \\
\hline $\mathrm{O}(6)-\mathrm{Fe}(5)-\mathrm{O}\left(8^{\mathrm{i}}\right)$ & $90.29(10)$ & $90.28(15)$ & $90.25(14)$ & $89.82(14)$ \\
\hline $\mathrm{O}(7)-\mathrm{Fe}(5)-\mathrm{O}\left(7^{\mathrm{i}}\right)$ & 180 & 180 & 180 & 180 \\
\hline $\mathrm{O}(7)-\mathrm{Fe}(5)-\mathrm{O}(8)$ & $90.07(11)$ & $89.88(16)$ & $90.04(14)$ & $90.07(15)$ \\
\hline $\mathrm{O}(7)-\mathrm{Fe}(5)-\mathrm{O}\left(8^{\mathrm{i}}\right)$ & $89.93(11)$ & $90.12(16)$ & $89.96(14)$ & $89.93(15)$ \\
\hline $\mathrm{O}(8)-\mathrm{Fe}(5)-\mathrm{O}\left(8^{\mathrm{i}}\right)$ & 180 & 180 & 180 & 180 \\
\hline
\end{tabular}




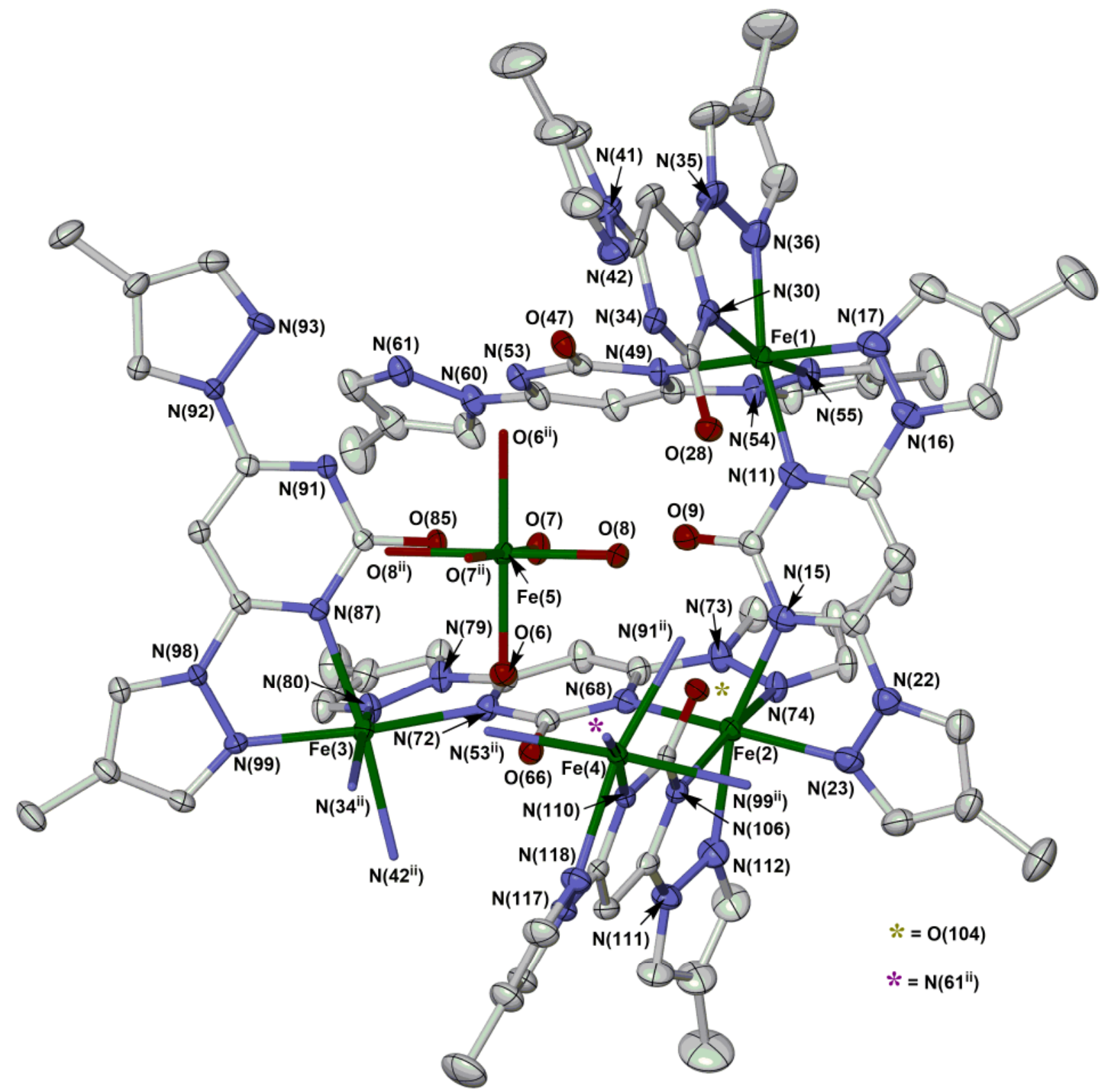

Figure S8 View of the half- $\left[\left\{\mathrm{Fe}\left(\mathrm{OH}_{2}\right)_{6}\right\} \subset \mathrm{Fe}_{8}\left(\mu-L^{2}\right)_{12}\right]^{7+}$ assembly in the asymmetric unit of $2 \cdot x \mathrm{MeCN} \cdot y \mathrm{Et} 2 \mathrm{O}$ at $125 \mathrm{~K}$, showing the numbering of the metal and ligand heteroatoms. Displacement ellipsoids are at the 50 $\%$ probability level, and $\mathrm{H}$ atoms are omitted for clarity. Symmetry code: (ii) $1-x, 1-y, 1-z$.

Color code: $\mathrm{C}$, white; Fe, green; $\mathrm{N}$, blue; $\mathrm{O}$, red. 
Table S6 Selected bond lengths and angles in the crystal structures of $2 \cdot x \mathrm{MeCN} \cdot y \mathrm{Et}_{2} \mathrm{O}\left(\AA{ }^{\circ},{ }^{\circ}\right)$. See Fig. S8 for the atom numbering scheme employed. Symmetry code: (ii) $1-x, 1-y, 1-z$.

\begin{tabular}{|c|c|c|c|}
\hline$T / \mathrm{K}$ & 125 & 200 & 250 \\
\hline $\mathrm{Fe}(1)-\mathrm{N}(11)$ & $2.089(3)$ & $2.149(3)$ & $2.157(3)$ \\
\hline $\mathrm{Fe}(1)-\mathrm{N}(17)$ & $2.045(4)$ & $2.175(3)$ & 2.191(4) \\
\hline $\mathrm{Fe}(1)-\mathrm{N}(30)$ & $2.081(3)$ & $2.142(3)$ & $2.151(3)$ \\
\hline $\mathrm{Fe}(1)-\mathrm{N}(36)$ & $2.054(4)$ & 2.191(3) & $2.214(4)$ \\
\hline $\mathrm{Fe}(1)-\mathrm{N}(49)$ & 2.104(3) & $2.174(3)$ & $2.182(3)$ \\
\hline $\mathrm{Fe}(1)-\mathrm{N}(55)$ & $2.049(3)$ & $2.159(3)$ & $2.185(4)$ \\
\hline $\mathrm{Fe}(2)-\mathrm{N}(15)$ & $2.173(3)$ & $2.174(3)$ & $2.179(3)$ \\
\hline $\mathrm{Fe}(2)-\mathrm{N}(23)$ & $2.142(4)$ & $2.176(3)$ & $2.178(4)$ \\
\hline $\mathrm{Fe}(2)-\mathrm{N}(68)$ & $2.172(3)$ & $2.190(3)$ & $2.187(3)$ \\
\hline $\mathrm{Fe}(2)-\mathrm{N}(74)$ & $2.135(3)$ & $2.170(3)$ & $2.176(4)$ \\
\hline $\mathrm{Fe}(2)-\mathrm{N}(106)$ & $2.146(3)$ & $2.155(3)$ & $2.160(3)$ \\
\hline $\mathrm{Fe}(2)-\mathrm{N}(112)$ & $2.186(3)$ & $2.205(3)$ & $2.220(4)$ \\
\hline $\mathrm{Fe}(3)-\mathrm{N}\left(34^{\mathrm{ii}}\right)$ & $2.212(3)$ & $2.204(3)$ & $2.206(3)$ \\
\hline $\mathrm{Fe}(3)-\mathrm{N}\left(42^{\mathrm{ii}}\right)$ & $2.172(3)$ & $2.182(3)$ & $2.181(4)$ \\
\hline $\mathrm{Fe}(3)-\mathrm{N}(72)$ & $2.148(3)$ & $2.162(3)$ & $2.170(3)$ \\
\hline $\mathrm{Fe}(3)-\mathrm{N}(80)$ & $2.189(3)$ & $2.200(3)$ & $2.196(4)$ \\
\hline $\mathrm{Fe}(3)-\mathrm{N}(87)$ & $2.168(3)$ & $2.172(2)$ & $2.171(3)$ \\
\hline $\mathrm{Fe}(3)-\mathrm{N}(93)$ & $2.137(3)$ & $2.164(3)$ & $2.167(3)$ \\
\hline $\mathrm{Fe}(4)-\mathrm{N}\left(53^{\mathrm{ii}}\right)$ & $2.209(3)$ & $2.199(3)$ & $2.202(3)$ \\
\hline $\mathrm{Fe}(4)-\mathrm{N}\left(61^{\mathrm{ii}}\right)$ & $2.166(3)$ & $2.175(3)$ & $2.169(3)$ \\
\hline $\mathrm{Fe}(4)-\mathrm{N}\left(91^{\mathrm{ii}}\right)$ & $2.163(3)$ & $2.161(2)$ & $2.166(3)$ \\
\hline $\mathrm{Fe}(4)-\mathrm{N}\left(99^{\mathrm{ii}}\right)$ & $2.212(3)$ & $2.203(3)$ & $2.201(3)$ \\
\hline $\mathrm{Fe}(4)-\mathrm{N}(110)$ & $2.192(3)$ & $2.203(2)$ & $2.206(3)$ \\
\hline $\mathrm{Fe}(4)-\mathrm{N}(118)$ & $2.212(3)$ & $2.208(3)$ & $2.205(4)$ \\
\hline $\mathrm{Fe}(5)-\mathrm{O}(6)$ & $1.996(2)$ & $1.996(2)$ & $1.995(3)$ \\
\hline $\mathrm{Fe}(5)-\mathrm{O}(7)$ & $1.994(2)$ & $2.001(2)$ & $2.001(3)$ \\
\hline $\mathrm{Fe}(5)-\mathrm{O}(8)$ & $1.995(2)$ & $1.997(2)$ & $1.992(3)$ \\
\hline $\mathrm{Fe}(1) \ldots \mathrm{Fe}(2)$ & $6.2410(7)$ & $6.2346(7)$ & $6.2506(9)$ \\
\hline $\mathrm{Fe}(1) \ldots \mathrm{Fe}\left(3^{\mathrm{ii}}\right)$ & $6.2738(8)$ & $6.2546(7)$ & $6.2665(9)$ \\
\hline $\mathrm{Fe}(1) \ldots \mathrm{Fe}\left(4^{\mathrm{ii}}\right)$ & $6.3372(7)$ & $6.3585(7)$ & $6.3568(8)$ \\
\hline $\mathrm{Fe}(2) \ldots \mathrm{Fe}(3)$ & $6.2767(8)$ & $6.3032(7)$ & $6.3077(8)$ \\
\hline $\mathrm{Fe}(2) \ldots \mathrm{Fe}(4)$ & $6.2599(8)$ & $6.2859(7)$ & $6.2881(8)$ \\
\hline $\mathrm{Fe}(3) \ldots \mathrm{Fe}\left(4^{\mathrm{ii}}\right)$ & $6.3188(7)$ & $6.3223(7)$ & $6.3142(8)$ \\
\hline $\mathrm{Fe}(1) \ldots \mathrm{Fe}\left(1^{\mathrm{ii}}\right)$ & $11.6168(11)$ & $11.5086(10)$ & $11.4807(13)$ \\
\hline $\mathrm{Fe}(2) \ldots \mathrm{Fe}\left(2^{\mathrm{ii}}\right)$ & $10.8357(11)$ & $10.9740(10)$ & $11.0058(12)$ \\
\hline $\mathrm{Fe}(3) \ldots \mathrm{Fe}\left(3^{\mathrm{ii}}\right)$ & $10.7535(11)$ & $10.7385(10)$ & $10.7302(12)$ \\
\hline $\mathrm{Fe}(4) \ldots \mathrm{Fe}\left(4^{\mathrm{ii}}\right)$ & $10.2929(11)$ & $10.3468(9)$ & $10.3829(12)$ \\
\hline $\mathrm{N}(11)-\mathrm{Fe}(1)-\mathrm{N}(17)$ & $77.45(13)$ & $74.56(11)$ & $74.25(13)$ \\
\hline $\mathrm{N}(11)-\mathrm{Fe}(1)-\mathrm{N}(30)$ & $105.35(12)$ & $109.48(10)$ & $109.80(12)$ \\
\hline $\mathrm{N}(11)-\mathrm{Fe}(1)-\mathrm{N}(36)$ & 167.97(14) & $163.43(11)$ & $162.90(14)$ \\
\hline $\mathrm{N}(11)-\mathrm{Fe}(1)-\mathrm{N}(49)$ & $102.73(12)$ & $106.30(10)$ & $106.58(12)$ \\
\hline $\mathrm{N}(11)-\mathrm{Fe}(1)-\mathrm{N}(55)$ & $88.30(12)$ & $88.60(11)$ & $88.46(14)$ \\
\hline $\mathrm{N}(17)-\mathrm{Fe}(1)-\mathrm{N}(30)$ & $91.16(13)$ & $92.40(11)$ & $92.39(13)$ \\
\hline $\mathrm{N}(17)-\mathrm{Fe}(1)-\mathrm{N}(36)$ & $90.78(14)$ & $89.17(12)$ & $88.93(15)$ \\
\hline $\mathrm{N}(17)-\mathrm{Fe}(1)-\mathrm{N}(49)$ & 168.63(13) & $165.25(11)$ & $164.53(14)$ \\
\hline $\mathrm{N}(17)-\mathrm{Fe}(1)-\mathrm{N}(55)$ & $91.38(13)$ & $90.95(11)$ & $90.43(14)$ \\
\hline $\mathrm{N}(30)-\mathrm{Fe}(1)-\mathrm{N}(36)$ & $77.05(13)$ & $73.68(11)$ & $73.31(13)$ \\
\hline $\mathrm{N}(30)-\mathrm{Fe}(1)-\mathrm{N}(49)$ & $99.67(11)$ & $100.95(10)$ & $101.59(12)$ \\
\hline $\mathrm{N}(30)-\mathrm{Fe}(1)-\mathrm{N}(55)$ & $166.34(13)$ & $161.85(11)$ & $161.60(14)$ \\
\hline $\mathrm{N}(36)-\mathrm{Fe}(1)-\mathrm{N}(49)$ & $88.31(14)$ & $88.62(11)$ & $88.78(14)$ \\
\hline $\mathrm{N}(36)-\mathrm{Fe}(1)-\mathrm{N}(55)$ & $89.50(14)$ & $88.55(12)$ & $88.57(15)$ \\
\hline $\mathrm{N}(49)-\mathrm{Fe}(1)-\mathrm{N}(55)$ & $77.28(12)$ & $74.42(10)$ & $74.22(13)$ \\
\hline $\mathrm{N}(15)-\mathrm{Fe}(2)-\mathrm{N}(23)$ & $74.32(12)$ & $73.60(10)$ & $73.55(13)$ \\
\hline $\mathrm{N}(15)-\mathrm{Fe}(2)-\mathrm{N}(68)$ & $109.16(12)$ & $110.26(10)$ & $110.01(12)$ \\
\hline $\mathrm{N}(15)-\mathrm{Fe}(2)-\mathrm{N}(74)$ & $83.43(12)$ & $83.74(11)$ & $83.58(14)$ \\
\hline $\mathrm{N}(15)-\mathrm{Fe}(2)-\mathrm{N}(106)$ & $107.66(11)$ & $107.52(10)$ & $108.03(12)$ \\
\hline $\mathrm{N}(15)-\mathrm{Fe}(2)-\mathrm{N}(112)$ & $163.76(13)$ & $161.69(11)$ & $161.31(14)$ \\
\hline
\end{tabular}


Table S6 continued.

\begin{tabular}{|c|c|c|c|}
\hline$T[\mathrm{~K}]$ & 125 & 200 & 250 \\
\hline $\mathrm{N}(23)-\mathrm{Fe}(2)-\mathrm{N}(68)$ & $165.72(12)$ & $164.26(11)$ & $164.12(14)$ \\
\hline $\mathrm{N}(23)-\mathrm{Fe}(2)-\mathrm{N}(74)$ & $92.03(12)$ & $91.47(11)$ & 91.11(14) \\
\hline $\mathrm{N}(23)-\mathrm{Fe}(2)-\mathrm{N}(106)$ & $91.29(12)$ & $91.75(10)$ & $91.68(13)$ \\
\hline $\mathrm{N}(23)-\mathrm{Fe}(2)-\mathrm{N}(112)$ & $89.52(13)$ & $88.13(11)$ & $87.82(14)$ \\
\hline $\mathrm{N}(68)-\mathrm{Fe}(2)-\mathrm{N}(74)$ & 74.92(11) & $74.15(10)$ & $74.25(13)$ \\
\hline $\mathrm{N}(68)-\mathrm{Fe}(2)-\mathrm{N}(106)$ & $100.50(11)$ & $101.20(10)$ & $101.49(12)$ \\
\hline $\mathrm{N}(68)-\mathrm{Fe}(2)-\mathrm{N}(112)$ & $86.13(12)$ & 87.04(11) & $87.54(13)$ \\
\hline $\mathrm{N}(74)-\mathrm{Fe}(2)-\mathrm{N}(106)$ & $168.90(12)$ & $168.74(11)$ & $168.38(13$ \\
\hline $\mathrm{N}(74)-\mathrm{Fe}(2)-\mathrm{N}(112)$ & $95.78(12)$ & $95.82(11)$ & $95.57(14)$ \\
\hline $\mathrm{N}(106)-\mathrm{Fe}(2)-\mathrm{N}(112)$ & $73.66(12)$ & $73.51(10)$ & $73.27(12)$ \\
\hline $\mathrm{N}\left(34^{\mathrm{ii}}\right)-\mathrm{Fe}(3)-\mathrm{N}\left(42^{\mathrm{ii}}\right)$ & 73.44(13) & $73.38(11)$ & $73.09(13)$ \\
\hline $\mathrm{N}\left(34^{4 i}\right)-\mathrm{Fe}(3)-\mathrm{N}(72)$ & $110.19(11)$ & $109.78(10)$ & $109.33(12)$ \\
\hline $\mathrm{N}\left(34^{\mathrm{ii}}\right)-\mathrm{Fe}(3)-\mathrm{N}(80)$ & $164.48(12)$ & $164.09(10)$ & $164.35(13)$ \\
\hline $\mathrm{N}\left(34^{\mathrm{ii}}\right)-\mathrm{Fe}(3)-\mathrm{N}(87)$ & $105.27(11)$ & $105.96(9)$ & $106.01(12)$ \\
\hline $\mathrm{N}\left(34^{\mathrm{ii}}\right)-\mathrm{Fe}(3)-\mathrm{N}(93)$ & $83.27(11)$ & $83.09(10)$ & $82.84(13)$ \\
\hline $\mathrm{N}\left(42^{\mathrm{ii}}\right)-\mathrm{Fe}(3)-\mathrm{N}(72)$ & $85.50(12)$ & $84.71(11)$ & $84.79(14)$ \\
\hline $\mathrm{N}\left(42^{\mathrm{ii}}\right)-\mathrm{Fe}(3)-\mathrm{N}(80)$ & $92.21(13)$ & $91.75(11)$ & $92.10(14)$ \\
\hline $\mathrm{N}\left(42^{\mathrm{ii}}\right)-\mathrm{Fe}(3)-\mathrm{N}(87)$ & $173.34(12)$ & $172.83(11)$ & $172.26(14)$ \\
\hline $\mathrm{N}\left(42^{\mathrm{ii}}\right)-\mathrm{Fe}(3)-\mathrm{N}(93)$ & $97.74(12)$ & $97.71(11)$ & $97.32(14)$ \\
\hline $\mathrm{N}(72)-\mathrm{Fe}(3)-\mathrm{N}(80)$ & 73.62(11) & $73.53(10)$ & $73.41(12)$ \\
\hline $\mathrm{N}(72)-\mathrm{Fe}(3)-\mathrm{N}(87)$ & $101.00(11)$ & $102.09(9)$ & $102.62(12)$ \\
\hline $\mathrm{N}(72)-\mathrm{Fe}(3)-\mathrm{N}(93)$ & $166.49(12)$ & $167.00(11)$ & $167.67(13)$ \\
\hline $\mathrm{N}(80)-\mathrm{Fe}(3)-\mathrm{N}(87)$ & $88.30(11)$ & $88.07(10)$ & $87.97(12)$ \\
\hline $\mathrm{N}(80)-\mathrm{Fe}(3)-\mathrm{N}(93)$ & $93.10(12)$ & $93.59(10)$ & $94.35(13)$ \\
\hline N(87)-Fe(3)-N(93) & $75.60(11)$ & $75.16(10)$ & $74.96(12)$ \\
\hline $\mathrm{N}\left(53^{\mathrm{ii}}\right)-\mathrm{Fe}(4)-\mathrm{N}\left(61^{\mathrm{ii}}\right)$ & $74.08(11)$ & $74.12(10)$ & $74.00(13)$ \\
\hline $\mathrm{N}\left(53^{3 i}\right)-\mathrm{Fe}(4)-\mathrm{N}\left(91^{\mathrm{ii}}\right)$ & $108.60(11)$ & $107.68(10)$ & 107.72(12) \\
\hline $\mathrm{N}\left(53^{\mathrm{ii}}\right)-\mathrm{Fe}(4)-\mathrm{N}\left(99^{\mathrm{ii}}\right)$ & $170.09(12)$ & $169.04(10)$ & $168.94(13)$ \\
\hline $\mathrm{N}\left(53^{\mathrm{ii}}\right)-\mathrm{Fe}(4)-\mathrm{N}(110)$ & $106.44(11)$ & 107.31(10) & $107.33(12)$ \\
\hline $\mathrm{N}\left(53^{\mathrm{ii}}\right)-\mathrm{Fe}(4)-\mathrm{N}(118)$ & $82.41(11)$ & $82.88(10)$ & $82.93(13)$ \\
\hline $\mathrm{N}\left(61^{\mathrm{ii}}\right)-\mathrm{Fe}(4)-\mathrm{N}\left(91^{\mathrm{ii}}\right)$ & $85.32(11)$ & $85.27(10)$ & $85.84(12)$ \\
\hline $\mathrm{N}\left(61^{\mathrm{ii}}\right)-\mathrm{Fe}(4)-\mathrm{N}\left(99^{\mathrm{ii}}\right)$ & $96.89(12)$ & $95.51(11)$ & $95.47(14)$ \\
\hline $\mathrm{N}\left(61^{\mathrm{ii}}\right)-\mathrm{Fe}(4)-\mathrm{N}(110)$ & $168.78(12)$ & $168.28(10)$ & $167.69(13)$ \\
\hline $\mathrm{N}\left(61^{\mathrm{ii}}\right)-\mathrm{Fe}(4)-\mathrm{N}(118)$ & $95.88(12)$ & $95.80(11)$ & $95.06(13)$ \\
\hline $\mathrm{N}\left(91^{\mathrm{ii}}\right)-\mathrm{Fe}(4)-\mathrm{N}\left(99^{\mathrm{ii}}\right)$ & $74.07(11)$ & $74.32(10)$ & $74.24(12)$ \\
\hline $\mathrm{N}\left(91^{\mathrm{ii}}\right)-\mathrm{Fe}(4)-\mathrm{N}(110)$ & $104.81(11)$ & 104.99(9) & $105.01(11)$ \\
\hline $\mathrm{N}\left(91^{\mathrm{ii}}\right)-\mathrm{Fe}(4)-\mathrm{N}(118)$ & $168.76(11)$ & $169.21(10)$ & $169.08(13)$ \\
\hline $\mathrm{N}\left(99^{\mathrm{ii}}\right)-\mathrm{Fe}(4)-\mathrm{N}(110)$ & $81.60(11)$ & $82.11(10)$ & $82.21(13)$ \\
\hline $\mathrm{N}\left(99^{\mathrm{ii}}\right)-\mathrm{Fe}(4)-\mathrm{N}(118)$ & $94.70(11)$ & $94.89(10)$ & $94.85(14)$ \\
\hline $\mathrm{N}(110)-\mathrm{Fe}(4)-\mathrm{N}(118)$ & $73.24(11)$ & $73.05(10)$ & $73.18(12)$ \\
\hline $\mathrm{O}(6)-\mathrm{Fe}(5)-\mathrm{O}\left(6^{\mathrm{ii}}\right)$ & 180 & 180 & 180 \\
\hline $\mathrm{O}(6)-\mathrm{Fe}(5)-\mathrm{O}(7)$ & $89.59(11)$ & $89.81(10)$ & $90.06(12)$ \\
\hline $\mathrm{O}(6)-\mathrm{Fe}(5)-\mathrm{O}\left(7^{\mathrm{ii}}\right)$ & $90.41(11)$ & $90.19(10)$ & $89.95(12)$ \\
\hline $\mathrm{O}(6)-\mathrm{Fe}(5)-\mathrm{O}(8)$ & $89.85(10)$ & $90.02(9)$ & $89.88(12)$ \\
\hline $\mathrm{O}(6)-\mathrm{Fe}(5)-\mathrm{O}\left(8^{\mathrm{ii}}\right)$ & $90.15(10)$ & $89.98(9)$ & $90.12(12)$ \\
\hline $\mathrm{O}(7)-\mathrm{Fe}(5)-\mathrm{O}\left(7^{\mathrm{ii}}\right)$ & 180 & 180 & 180 \\
\hline $\mathrm{O}(7)-\mathrm{Fe}(5)-\mathrm{O}(8)$ & $90.58(10)$ & $90.31(9)$ & $90.46(12)$ \\
\hline $\mathrm{O}(7)-\mathrm{Fe}(5)-\mathrm{O}\left(8^{\mathrm{ii}}\right)$ & $89.42(10)$ & $89.69(9)$ & $89.54(12)$ \\
\hline $\mathrm{O}(8)-\mathrm{Fe}(5)-\mathrm{O}\left(8^{\mathrm{ii}}\right)$ & 180 & 180 & 180 \\
\hline
\end{tabular}


Table S7 Dihedral angles between the least squares planes of the bidentate pyrazolyl-pyrimidonate ligand fragments coordinated to each vertex of the cubane assembly in $1 \cdot 6 \mathrm{MeCN}\left({ }^{\circ}\right)$. See Fig. S7 for the atom numbering scheme. Angles involving disordered pyrazolyl groups are averaged over both disorder orientations. Symmetry code: (i) $3 / 2-x, 1 / 2-y, 1-z$.

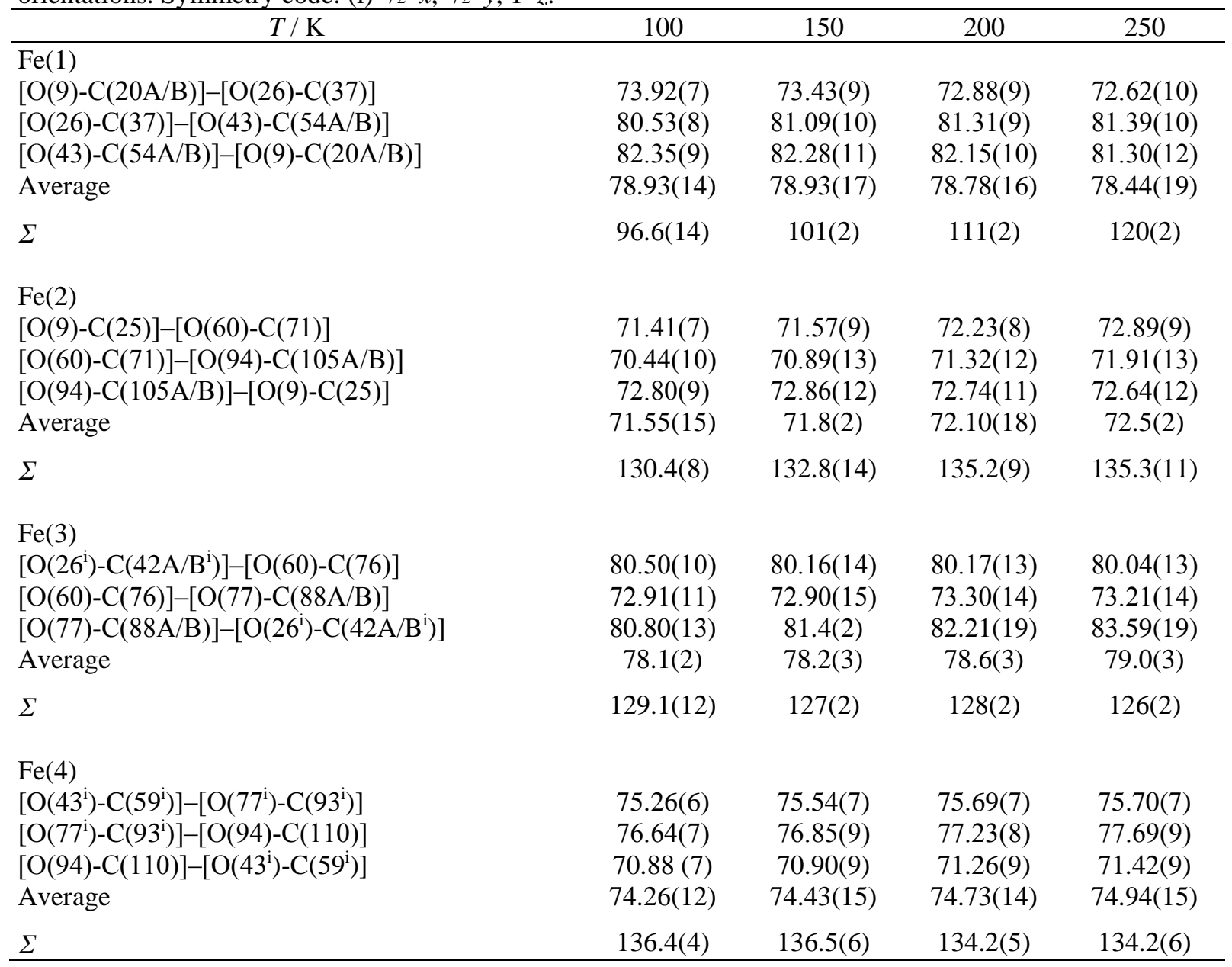

The planes of the bidentate ligands in an "ideal" octahedral tris-chelate center should be at right angles to each other. By that measure, the ligand geometries about $\mathrm{Fe}(2)$ and $\mathrm{Fe}(4)$ are significantly more distorted than about $\mathrm{Fe}(1)$ and $\mathrm{Fe}(3)$.

Values for $\Sigma$ are also given for comparison with Table S8, but the interpretation of the averaged $\Sigma$ values for the most disordered vertices $\mathrm{Fe}(1)$ and $\mathrm{Fe}(3)$ is not straightforward. See Table S8 for a discussion of this parameter. 
Table S8 Dihedral angles between the least squares planes of the bidentate pyrazolyl-pyrimidonate ligand fragments coordinated to each vertex of the cubane assembly in $2 \cdot x \mathrm{MeCN} \cdot y \mathrm{Et}_{2} \mathrm{O}\left({ }^{\circ}\right)$. See Fig. S8 for the atom numbering scheme. Symmetry code: (ii) $1-x, 1-y, 1-z$.

\begin{tabular}{lccc}
\hline \multicolumn{1}{c}{$T / \mathrm{K}$} & 125 & 200 & 250 \\
\hline $\mathrm{Fe}(1)$ & & & \\
{$[\mathrm{O}(9)-\mathrm{C}(20)]-[\mathrm{O}(28)-\mathrm{C}(39)]$} & $86.69(7)$ & $84.89(6)$ & $84.54(8)$ \\
{$[\mathrm{O}(28)-\mathrm{C}(39)]-[\mathrm{O}(47)-\mathrm{C}(58)]$} & $86.87(6)$ & $86.87(6)$ & $86.85(8)$ \\
{$[\mathrm{O}(47)-\mathrm{C}(58)]-[\mathrm{O}(9)-\mathrm{C}(20)]$} & $84.12(5)$ & $83.46(4)$ & $83.46(5)$ \\
Average & $85.89(10)$ & $85.07(09)$ & $84.95(12)$ \\
$\Sigma$ & $83.2(4)$ & $102.5(4)$ & $104.3(5)$ \\
& & & \\
$\mathrm{Fe}(2)$ & & & \\
{$[\mathrm{O}(9)-\mathrm{C}(26)]-[\mathrm{O}(66)-\mathrm{C}(77)]$} & $74.24(5)$ & $74.39(4)$ & $74.20(5)$ \\
{$[\mathrm{O}(66)-\mathrm{C}(77)]-[\mathrm{O}(104)-\mathrm{C}(115)]$} & $75.02(6)$ & $76.96(6)$ & $78.01(7)$ \\
{$[\mathrm{O}(104)-\mathrm{C}(115)]-[\mathrm{O}(9)-\mathrm{C}(26)]$} & $88.25(7)$ & $87.04(6)$ & $86.51(8)$ \\
$\mathrm{Average}$ & $79.17(10)$ & $79.46(9)$ & $79.57(12)$ \\
$\Sigma$ & $114.4(4)$ & $117.9(4)$ & $117.9(5)$ \\
& & & \\
$\mathrm{Fe}(3)$ & & & \\
{$\left[\mathrm{O}\left(28^{\mathrm{ii}}\right)-\mathrm{C}\left(45^{\mathrm{ii}}\right)\right]-[\mathrm{O}(66)-\mathrm{C}(83)]$} & $82.98(5)$ & $82.15(5)$ & $81.29(6)$ \\
{$[\mathrm{O}(66)-\mathrm{C}(83)]-[\mathrm{O}(85)-\mathrm{C}(96)]$} & $80.46(5)$ & $80.02(5)$ & $79.65(6)$ \\
{$[\mathrm{O}(85)-\mathrm{C}(96)]-\left[\mathrm{O}\left(28^{\mathrm{ii}}\right)-\mathrm{C}\left(45^{\mathrm{ii}}\right)\right]$} & $66.42(5)$ & $65.86(5)$ & $65.45(6)$ \\
$\mathrm{Average}$ & $76.62(9)$ & $76.01(9)$ & $75.46(10)$ \\
$\Sigma$ & $119.8(4)$ & $122.9(4)$ & $124.7(4)$ \\
& & & \\
$\mathrm{Fe}(4)$ & & & \\
{$\left[\mathrm{O}\left(47^{\mathrm{ii}}\right)-\mathrm{C}\left(64^{\mathrm{ii}}\right)\right]-\left[\mathrm{O}\left(85^{\mathrm{ii}}\right)-\mathrm{C}\left(102^{\mathrm{ii}}\right)\right]$} & $74.08(5)$ & $75.11(5)$ & $76.20(6)$ \\
{$\left[\mathrm{O}\left(85^{\mathrm{ii}}\right)-\mathrm{C}\left(102^{\mathrm{ii}}\right)\right]-[\mathrm{O}(104)-\mathrm{C}(121)]$} & $67.64(5)$ & $67.28(5)$ & $67.31(6)$ \\
{$[\mathrm{O}(104)-\mathrm{C}(121)]-\left[\mathrm{O}\left(47^{\mathrm{ii}}\right)-\mathrm{C}\left(64^{\mathrm{ii}}\right)\right]$} & $68.56(5)$ & $69.49(5)$ & $70.00(6)$ \\
$\mathrm{Average}$ & $70.09(9)$ & $70.63(9)$ & $71.17(10)$ \\
$\Sigma$ & $136.6(4)$ & $134.4(3)$ & $133.0(4)$ \\
\hline
\end{tabular}

The planes of the bidentate ligands in an "ideal" octahedral tris-chelate center should be at right angles to each other. By that measure, the ligand geometries about $\mathrm{Fe}(2), \mathrm{Fe}(3)$ and $\mathrm{Fe}(4)$ are significantly more distorted than about $\mathrm{Fe}(1)$. This difference is more clear cut than in $1 \cdot 6 \mathrm{MeCN}$ (Table S7).

The $\Sigma$ parameter is calculated from the $\mathrm{N}-\mathrm{Fe}-\mathrm{N}$ bond angles, as a measure of the deviation of the $\mathrm{FeN}_{6}$ coordination sphere from an ideal octahedral geometry (a perfect octahedron gives $\Sigma=0$ ). ${ }^{2}$ Thus, the less twisted arrangement of ligands about $\mathrm{Fe}(1)$ is also reflected in its more regular coordination geometry at 200 and $250 \mathrm{~K}$, compared to the other vertices.

The lower value of $\Sigma$ for $\mathrm{Fe}(1)$ at $125 \mathrm{~K}$ is consistent with its high $\rightarrow$ low spin transition. ${ }^{2}$ 
Table S9 Hydrogen bond parameters in $\mathbf{1} \cdot 6 \mathrm{CH}_{3} \mathrm{CN}$ at $100 \mathrm{~K}\left(\AA{ }^{\circ}\right)$. See Fig. S7 for the atom numbering scheme employed. Symmetry code: (i) ${ }^{3} / 2-x,{ }^{1} / 2-y, 1-z$.

\begin{tabular}{lllll}
\hline & $\mathrm{D}-\mathrm{H}$ & $\mathrm{H} \ldots \mathrm{A}$ & $\mathrm{D} \ldots \mathrm{A}$ & $\mathrm{D}-\mathrm{H} \ldots \mathrm{A}$ \\
\hline $\mathrm{O}(6)-\mathrm{H}(6 \mathrm{~A}) \ldots \mathrm{O}(60)$ & $0.900(10)$ & $1.711(11)$ & $2.611(3)$ & $177(4)$ \\
$\mathrm{O}(6)-\mathrm{H}(6 \mathrm{~B}) \ldots \mathrm{O}\left(43^{\mathrm{i}}\right)$ & $0.900(10)$ & $1.699(13)$ & $2.592(3)$ & $172(4)$ \\
$\mathrm{O}(7)-\mathrm{H}(7 \mathrm{~A}) \ldots \mathrm{O}(9)$ & $0.895(10)$ & $1.727(14)$ & $2.614(4)$ & $170(4)$ \\
$\mathrm{O}(7)-\mathrm{H}(7 \mathrm{~B}) \ldots \mathrm{O}(77)$ & $0.905(10)$ & $1.685(11)$ & $2.588(4)$ & $176(4)$ \\
$\mathrm{O}(8)-\mathrm{H}(8 \mathrm{~A}) \ldots \mathrm{O}(94)$ & $0.901(10)$ & $1.701(11)$ & $2.599(4)$ & $174(4)$ \\
$\mathrm{O}(8)-\mathrm{H}(8 \mathrm{~B}) \ldots \mathrm{O}(26)$ & $0.899(10)$ & $1.737(19)$ & $2.599(4)$ & $160(4)$ \\
\hline
\end{tabular}

Table S10 Temperature dependence of the O...O distances for the intramolecular hydrogen bonds in 1.6MeCN $(\AA){ }^{\text {a }}$ See Fig. S7 for the atom numbering scheme employed. Symmetry code:

(i) ${ }^{3} / 2-x, 1 / 2-y, 1-z$.

\begin{tabular}{lcccc}
\hline$T / \mathrm{K}$ & 100 & 150 & 200 & 250 \\
\hline $\mathrm{O}(6) \ldots \mathrm{O}(60)$ & $2.611(3)$ & $2.601(5)$ & $2.610(4)$ & $2.622(5)$ \\
$\mathrm{O}(6) \ldots \mathrm{O}\left(43^{\mathrm{i}}\right)$ & $2.592(3)$ & $2.587(5)$ & $2.589(4)$ & $2.588(5)$ \\
$\mathrm{O}(7) \ldots \mathrm{O}(9)$ & $2.614(4)$ & $2.610(6)$ & $2.623(5)$ & $2.648(6)$ \\
$\mathrm{O}(7) \ldots \mathrm{O}(77)$ & $2.588(4)$ & $2.583(5)$ & $2.580(5)$ & $2.571(5)$ \\
$\mathrm{O}(8) \ldots \mathrm{O}(94)$ & $2.599(4)$ & $2.595(5)$ & $2.596(5)$ & $2.615(5)$ \\
$\mathrm{O}(8) \ldots \mathrm{O}(26)$ & $2.599(4)$ & $2.588(5)$ & $2.610(5)$ & $2.615(5)$ \\
\hline
\end{tabular}

${ }^{\text {a }}$ The refinements at $T \geq 150 \mathrm{~K}$ do not include $\mathrm{H}$ atoms at $\mathrm{O}(6), \mathrm{O}(7)$ and $\mathrm{O}(8)$.

Only the $\mathrm{O}(7) \ldots \mathrm{O}(9)$ hydrogen bond shows a significant variation over this temperature range, within crystallographic experimental error.

Table S11 Temperature dependence of the O...O distances for the intramolecular hydrogen bonds in $2 \cdot x \mathrm{MeCN}_{y} y \mathrm{Et}_{2} \mathrm{O}(\AA)$. ${ }^{\mathrm{a}}$ See Fig. S8 for the atom numbering scheme employed. Symmetry code:

(ii) $1-x, 1-y, 1-z$.

\begin{tabular}{lccc}
\hline$T / \mathrm{K}$ & 125 & 200 & 250 \\
\hline $\mathrm{O}(6) \ldots \mathrm{O}(66)$ & $2.584(4)$ & $2.607(3)$ & $2.613(4)$ \\
$\mathrm{O}(6) \ldots \mathrm{O}\left(47^{\mathrm{ii}}\right)$ & $2.577(3)$ & $2.588(3)$ & $2.589(4)$ \\
$\mathrm{O}(7) \ldots \mathrm{O}(9)$ & $2.584(4)$ & $2.600(3)$ & $2.609(4)$ \\
$\mathrm{O}(7) \ldots \mathrm{O}(85)$ & $2.587(3)$ & $2.598(3)$ & $2.598(4)$ \\
$\mathrm{O}(8) \ldots \mathrm{O}(104)$ & $2.593(3)$ & $2.594(3)$ & $2.599(4)$ \\
$\mathrm{O}(8) \ldots \mathrm{O}(28)$ & $2.605(4)$ & $2.611(3)$ & $2.614(4)$ \\
\hline
\end{tabular}

${ }^{a}$ The refinements do not include $\mathrm{H}$ atoms at $\mathrm{O}(6), \mathrm{O}(7)$ and $\mathrm{O}(8)$.

Only the $\mathrm{O}(6) \ldots \mathrm{O}(66)$ and $\mathrm{O}(7) \ldots \mathrm{O}(9)$ hydrogen bonds show significant variation over this temperature range, within crystallographic experimental error. 
Table S12 Bond valence sum (BVS) values for 1.6MeCN. These were calculated using parameters for highspin $\mathrm{Fe}-\mathrm{N}$ and $\mathrm{Fe}-\mathrm{O}$ bonds, unless otherwise stated. ${ }^{7}$ Separate values are given for each ligand disorder site about an Fe atom, where relevant. See Fig. S7 for the atom numbering scheme.

\begin{tabular}{lcccc}
\hline$T / \mathrm{K}$ & 100 & 150 & 200 & 250 \\
\hline BVS $\{\mathrm{Fe}(1)\}$ & $1.56(1)-1.89(1)^{\mathrm{a}}$ & $-^{\mathrm{b}}$ & $-^{\mathrm{b}}$ & $2.01(2)-2.44(2)$ \\
BVS $\{\mathrm{Fe}(2)\}$ & $2.10(1)-2.11(1)$ & $2.00(1)-2.05(1)$ & $1.96(1)-1.98(1)$ & $1.94(1)-1.99(1)$ \\
BVS $\{\mathrm{Fe}(3)\}$ & $1.81(1)-2.03(1)$ & $1.80(2)-2.04(2)$ & $1.81(2)-2.09(2)$ & $1.83(2)-2.21(2)$ \\
BVS $\{\mathrm{Fe}(4)\}$ & $1.926(8)$ & $1.94(1)$ & $1.93(1)$ & $1.91(1)$ \\
BVS $\{\mathrm{Fe}(5)\}$ & $3.081(6)$ & $3.028(9)$ & $3.035(8)$ & $3.031(8)$ \\
\hline
\end{tabular}

${ }^{\mathrm{a}}$ Calculated using BVS parameters for low-spin $\mathrm{Fe}-\mathrm{N}$ bonds. ${ }^{\mathrm{b}}$ Iron atom is in a mixed spin-state population at this temperature, so accurate BVS data are not expected.

Table S13 Bond valence sum (BVS) values for $2 \cdot x \mathrm{MeCN} \cdot y \mathrm{Et}_{2} \mathrm{O}$. These were calculated using parameters for high-spin $\mathrm{Fe}-\mathrm{N}$ and $\mathrm{Fe}-\mathrm{O}$ bonds, unless otherwise stated. ${ }^{7}$ See Fig. S8 for the atom numbering scheme.

\begin{tabular}{lccc}
$T / \mathrm{K}$ & 125 & 200 & 250 \\
\hline BVS $\{\mathrm{Fe}(1)\}$ & $2.662(8)\left[1.513(8)^{\mathrm{a}}\right]^{\mathrm{b}}$ & $2.060(7)$ & $1.979(9)$ \\
BVS $\{\mathrm{Fe}(2)\}$ & $2.094(7)$ & $1.986(7)$ & $1.960(9)$ \\
BVS $\{\mathrm{Fe}(3)\}$ & $2.029(7)$ & $1.974(7)$ & $1.967(8)$ \\
BVS $\{\mathrm{Fe}(4)\}$ & $1.914(7)$ & $1.917(7)$ & $1.917(8)$ \\
BVS $\{\mathrm{Fe}(5)\}$ & $3.101(5)$ & $3.081(5)$ & $3.094(7)$ \\
\hline
\end{tabular}

${ }^{a}$ Alternative value calculated using BVS parameters for low-spin Fe-N bonds. ${ }^{b}$ Iron atom is in a mixed spinstate population at this temperature, so accurate BVS data are not expected.

A bond valence sum (BVS) is equivalent to the oxidation state of a metal ion, calculated from its bond lengths using standardized parameters characteristic for each metal ion and donor atom (eq S1). ${ }^{3}$

$$
\mathrm{BVS}=\sum \exp \left(\left(r_{o}-r\right) / b\right)
$$

where $r$ is the measured bond length between the two given atoms; $r_{0}$ is the bond valence parameter for that bond; and $b \approx 0.37$. Values of $r_{0}$ and $b$ have been tabulated for a wide range of metal/donor atom combinations. ${ }^{4}$ This approach is widely used for the calculation of oxidation states in inorganic minerals, but the concept has also been extended to small molecules and metalloproteins.

The assignments of $\mathrm{Fe}(2)-\mathrm{Fe}(4)$ as $\mathrm{Fe}(\mathrm{II})$ centers, and of $\mathrm{Fe}(5)$ as a $\mathrm{Fe}(\mathrm{III})$ site, are obvious in both structures. BVS data for $\mathrm{Fe}(1)$ are less clear cut because of its changing spin-state population; and, because BVS parameters for low-spin metal ions are less developed than for high-spin ions (which are prevalent in minerals and metalloproteins). However, the BVS values for $\mathrm{Fe}(1)$ when it is predominantly high-spin, at $250 \mathrm{~K}$ for $1 \cdot 6 \mathrm{MeCN}$ and at 200 or $250 \mathrm{~K}$ for $2 \cdot x \mathrm{MeCN} \cdot y \mathrm{Et}_{2} \mathrm{O}$, are more consistent with a +2 than a +3 oxidation state. 


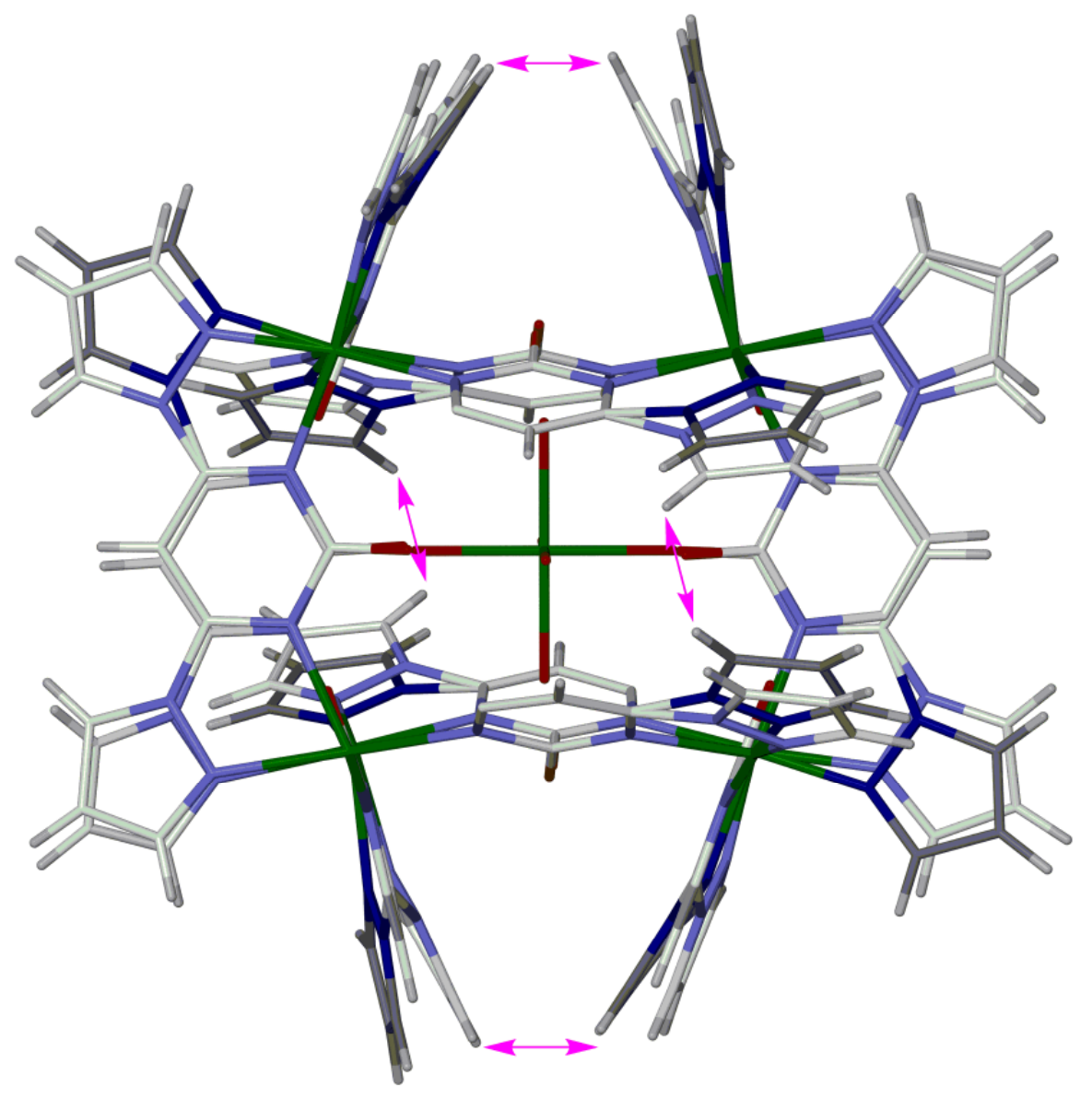

Figure S9 View of the $\left[\left\{\mathrm{Fe}\left(\mathrm{OH}_{2}\right)_{6}\right\} \subset \mathrm{Fe}_{8}\left(\mu-L^{1}\right)_{12}\right]^{7+}$ assembly in $\mathbf{1} \cdot 6 \mathrm{CH}_{3} \mathrm{CN}$, highlighting the intramolecular steric clashes giving rise to the pyrazolyl group disorder (Fig. S7). Some crystallographically ordered atoms that obscure the view of the disordered groups are omitted from the image.

Color code: $\mathrm{C}$, white; $\mathrm{Fe}$, green; $\mathrm{N}$, blue; $\mathrm{O}$, red.

The 'A' and ' $\mathrm{B}$ ' disorder orientations of each residue have pale and dark coloration, respectively. The pink arrows highlight $\mathrm{H}$...H distances of 1.9-2.0 A between A and B disorder sites on neighboring pyrazolyl groups, which cannot therefore co-exist in the same molecule. 


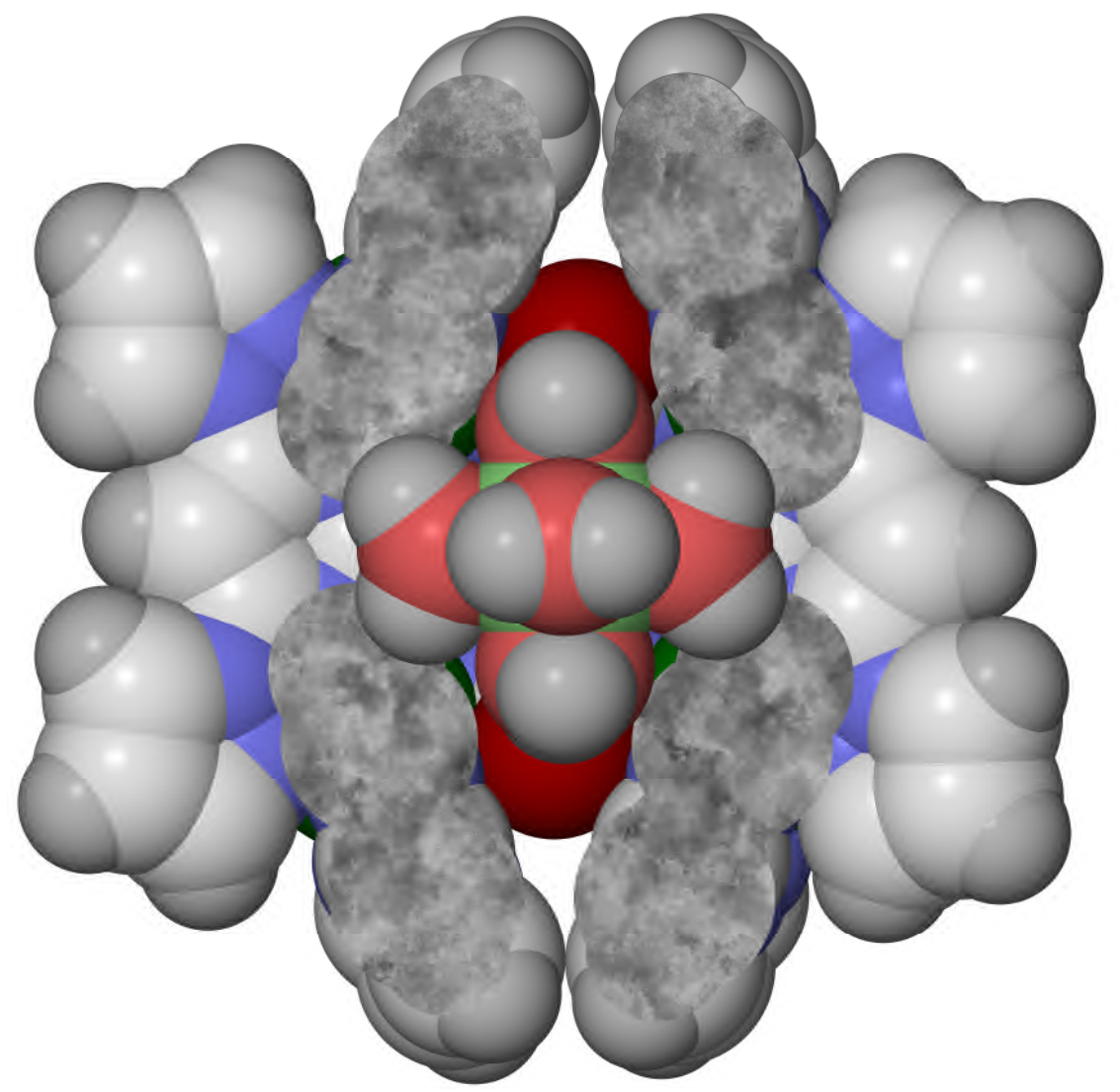

Figure S10 Cut-away space-filling plot of the $\left[\left\{\mathrm{Fe}\left(\mathrm{OH}_{2}\right)_{6}\right\} \subset \mathrm{Fe}_{8}\left(\mu-L^{1}\right)_{12}\right]^{7+}$ assembly in $\mathbf{1} \cdot 6 \mathrm{MeCN}$ at $100 \mathrm{~K}$. For clarity, the encapsulated $\left[\mathrm{Fe}\left(\mathrm{OH}_{2}\right)_{6}\right]^{3+}$ cation is shown with pale coloration. The view is the same as in Figure 2 of the main article.

Color code: $\mathrm{C}$, white; $\mathrm{H}$, pale grey; Fe, dark or pale green; N, blue; O, dark or pale red. 

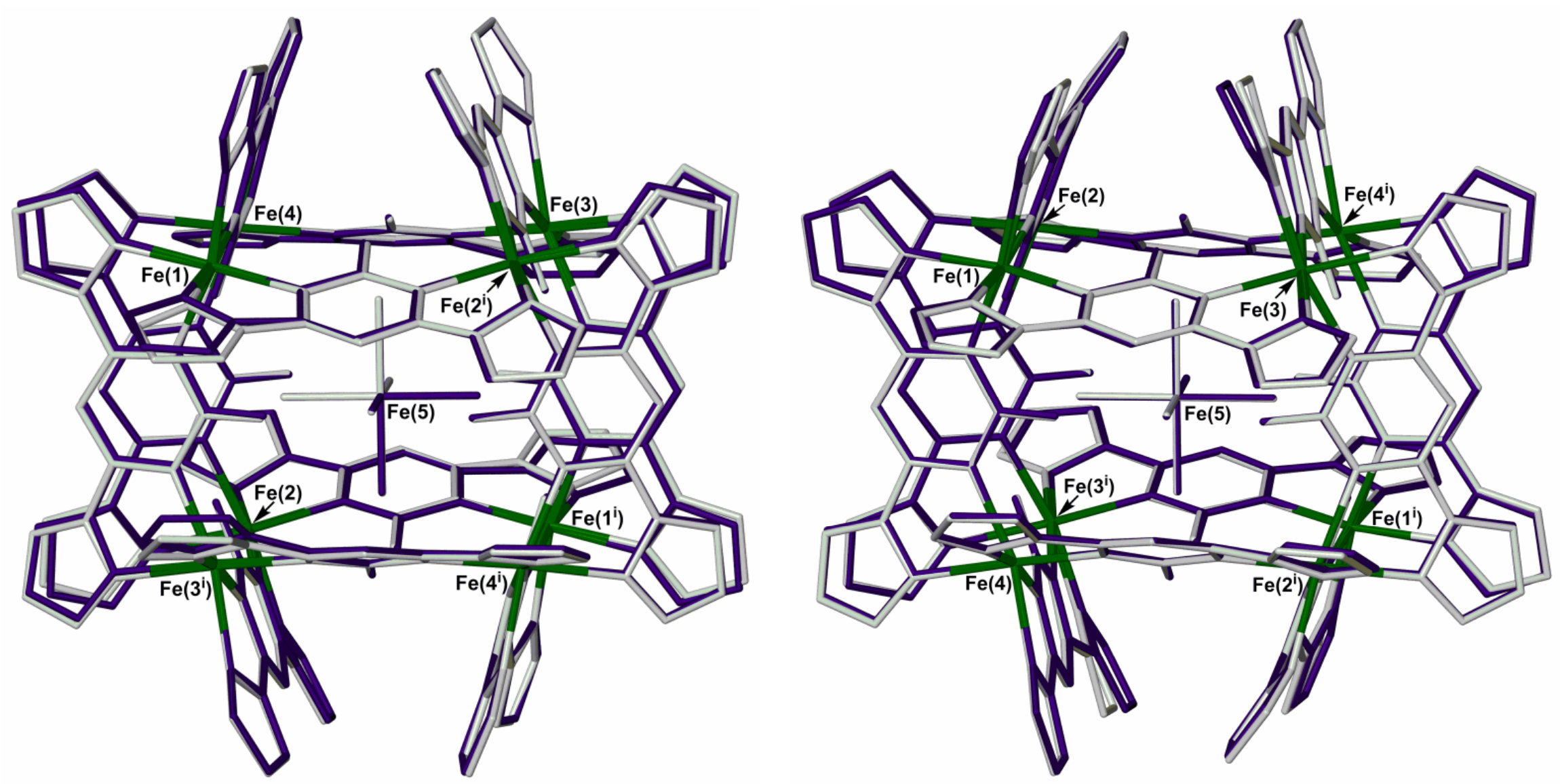

Figure S11 Overlays of the high-spin (white, $250 \mathrm{~K}$ ) and low-spin (purple, $100 \mathrm{~K}$ ) structures of the $\left[\left\{\mathrm{Fe}\left(\mathrm{OH}_{2}\right)_{6}\right\} \subset \mathrm{Fe}_{8}\left(\mu-L^{1}\right)_{12}\right]^{7+}$ assembly in $\mathbf{1} \cdot 6 \mathrm{CH} \mathrm{CHN}_{3} \mathrm{C}$, shown from two right-angled views. For clarity $\mathrm{H}$ atoms are omitted, and only one orientation of the disordered pyrazolyl residues is shown. The Fe ions of the cubane cage are highlighted in green, for clarity; $\mathrm{Fe}(1)$ and $\mathrm{Fe}\left(1^{\mathrm{i}}\right)$ are the SCO-active vertices. Symmetry code: $(\mathrm{i}){ }^{3} / 2-x, 1 / 2-y, 1-z$.

The displacement of the iron atoms between the two structures is small, at $\leq 0.09 \AA$. However, non-H atoms in the pyrazolyl groups are displaced by up to $0.50 \AA$ between the two temperatures. These rearrangements may be necessary to accommodate the contraction at $\mathrm{Fe}(1)$ and $\mathrm{Fe}\left(1^{\mathrm{i}}\right)$ that accompanies their SCO, without changing the volume of the $\left[\mathrm{Fe} 8\left(\mu-L^{1}\right)_{12}\right]^{4+}$ cage [which is dictated by the $\left[\mathrm{Fe}\left(\mathrm{OH}_{2}\right)_{6}\right]^{3+}$ template]. 


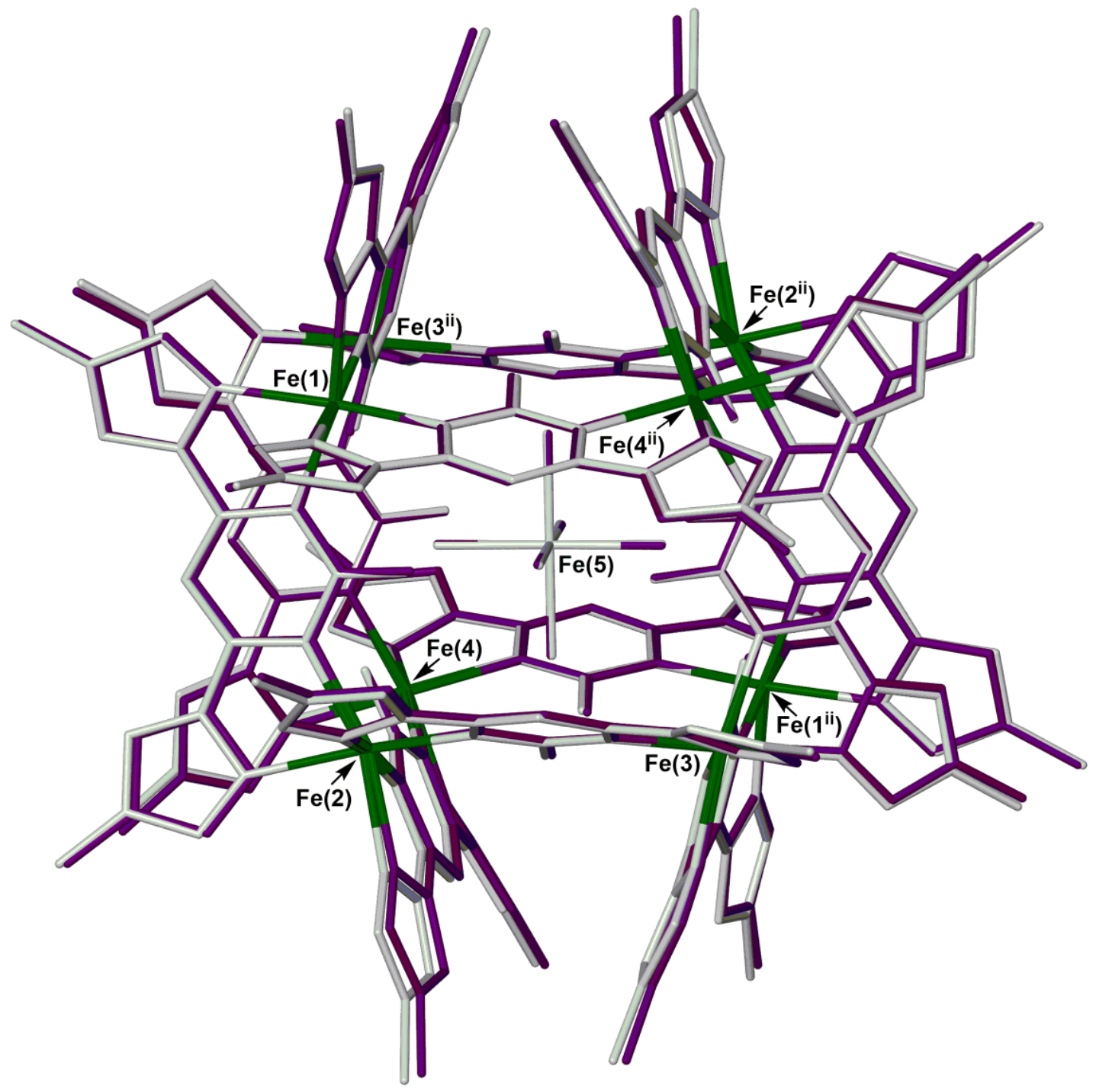

Figure S12 Overlay of the high-spin (white, $250 \mathrm{~K}$ ) and low-spin (purple, $125 \mathrm{~K}$ ) structures of the $\left[\left\{\mathrm{Fe}\left(\mathrm{OH}_{2}\right)_{6}\right\} \subset \mathrm{Fe}_{8}\left(\mu-L^{2}\right)_{12}\right]^{7+}$ assembly in $2 \cdot x \mathrm{MeCN} \cdot y \mathrm{Et}_{2} \mathrm{O}$. Details as for Fig. S11; $\mathrm{Fe}(1)$ and $\mathrm{Fe}\left(1^{\mathrm{i}}\right)$ are the SCO-active vertices. Symmetry code: (ii) $1-x, 1-y, 1-z$.

The heavy atom displacements between these two temperatures are smaller than for $1 \cdot 6 \mathrm{MeCN}$, at $\leq 0.35 \AA$. That may reflect that the spin-crossover at $\mathrm{Fe}(1)$ is still incomplete at $125 \mathrm{~K}$. 

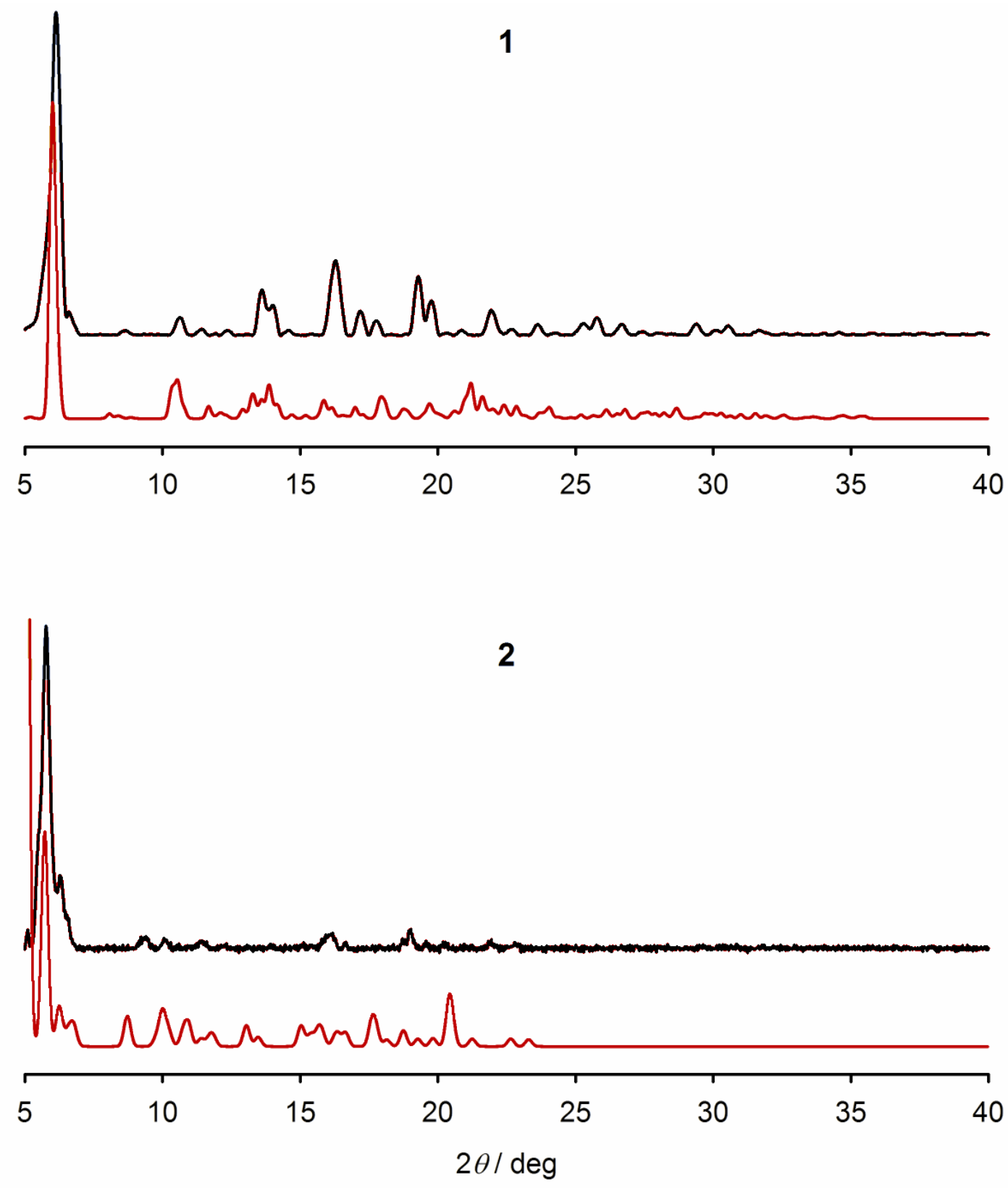

Figure S13 Black: X-ray powder diffraction data for air-dried samples of $\mathbf{1}$ (top) and $\mathbf{2}$ (bottom) at room temperature. Red: simulated powder patterns from $1 \cdot 6 \mathrm{MeCN}$ and $2 \cdot x \mathrm{MeCN}_{y} \cdot y \mathrm{Et}_{2} \mathrm{O}$ at $250 \mathrm{~K}$.

Desolvation of $1 \cdot 6 \mathrm{CH}_{3} \mathrm{CN}$ and $2 \cdot x \mathrm{MeCN} \cdot y \mathrm{Et}_{2} \mathrm{O}$ leads to a reduction in their long-range crystallinity, but the main features of both simulated powder patterns are retained in the experimental data. We conclude that the original local lattice structure is retained in both dried materials.

Preferred orientation effects can also lead to differences in the measured and simulated peak intensities in this type of measurement. 


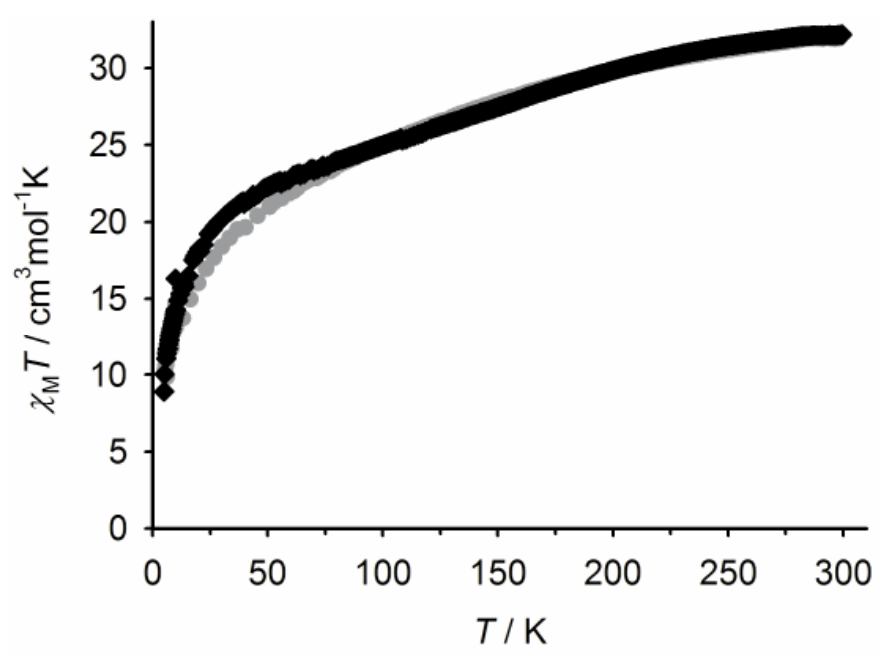

Figure S14 Variable temperature magnetic susceptibility data for freshly prepared $1 \cdot 6 \mathrm{CH}_{3} \mathrm{CN}$ (black) and desolvated 1 (gray). The black data are the same as Figure 4 of the main article.

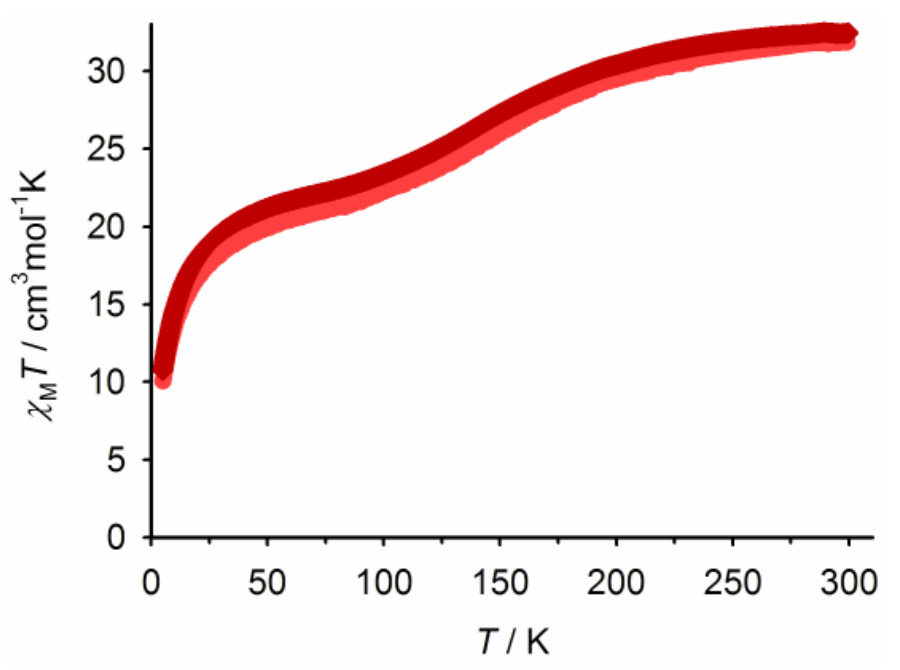

Figure S15 Variable temperature magnetic susceptibility data for freshly prepared $2 \cdot x \mathrm{MeCN} \cdot y \mathrm{Et}_{2} \mathrm{O}$ (dark red) and desolvated $\mathbf{2}$ (pale red). The dark red data are the same as Figure 4 of the main article.

The $\chi_{\mathrm{M}} T v s T$ curve from dried $\mathbf{1}$ has a less defined low-temperature plateau than for polycrystalline $1 \cdot 6 \mathrm{CH}_{3} \mathrm{CN}$. In other respects, the magnetic data from dried samples of $\mathbf{1}$ and $\mathbf{2}$ are almost identical to their freshly prepared samples. That is consistent with the $\left[\left\{\mathrm{Fe}\left(\mathrm{OH}_{2}\right)_{6}\right\} \subset \mathrm{Fe}_{8}(\mu-\mathrm{L})_{12}\right]^{7+}$ assemblies retaining their integrity in dried samples of $\mathbf{1}$ and $\mathbf{2}$. 

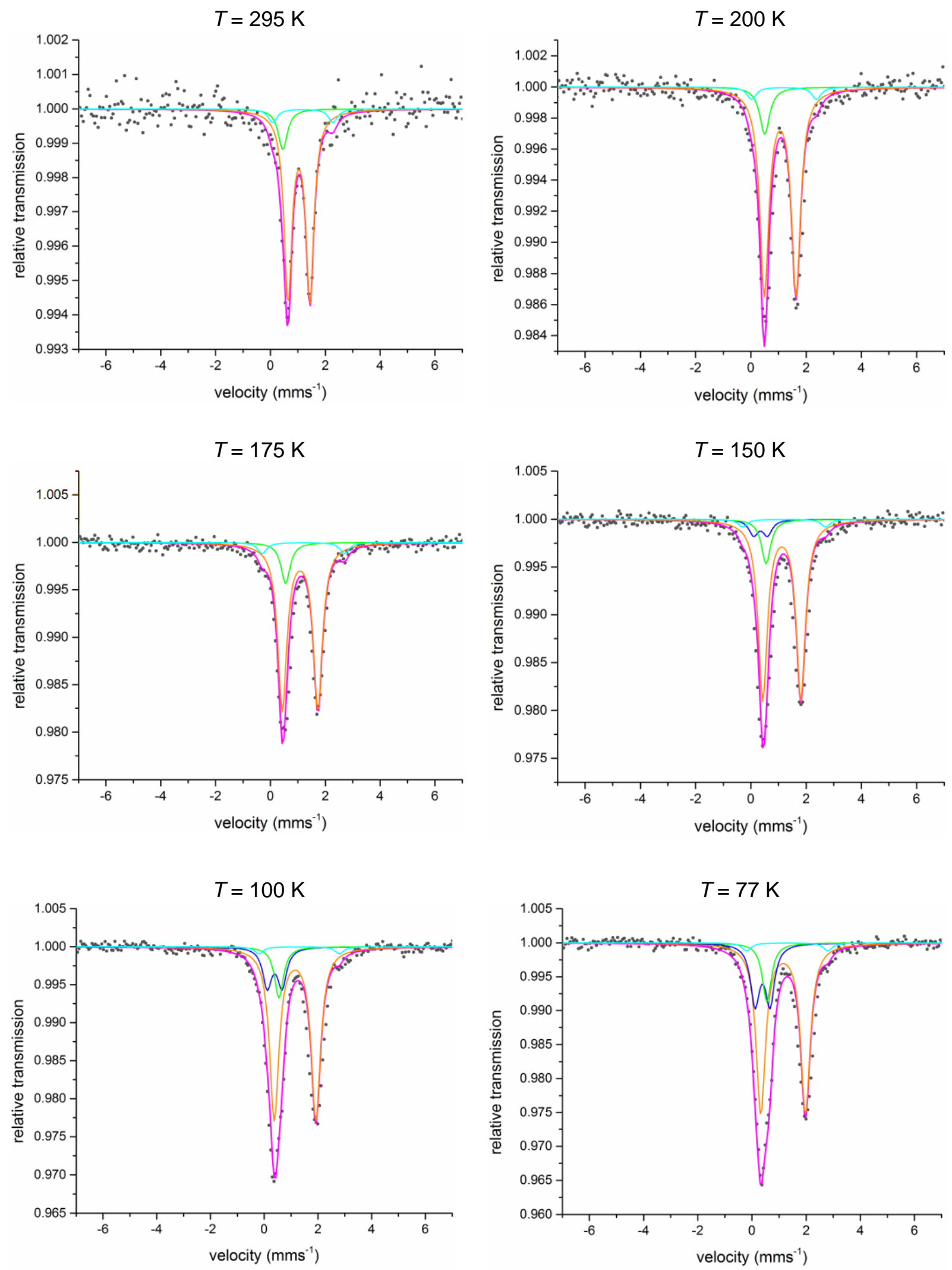

Figure S16 Measured and simulated Mössbauer spectra of 1. The graph at $T=77 \mathrm{~K}$ is the same as Figure 6 of the main article.

The simulations are in pink, with the components of those simulations color coded as follows: high-spin iron(II) (orange); low-spin iron(II) (blue); high-spin iron(III) (green); and iron(II) impurity (cyan). 
Table S14 Parameters from the Mössbauer spectrum simulations in Figure S16.

\begin{tabular}{|c|c|c|c|c|}
\hline & High-spin iron(II) & Low-spin iron(II) & High-spin iron(III) & Impurity iron(II) \\
\hline \multicolumn{5}{|l|}{$T=295 \mathrm{~K}$} \\
\hline$\delta / \mathrm{mms}^{-1}$ & $1.04 \pm 0.04$ & 0 & $0.45 \pm 0.04$ & $1.18 \pm 0.04$ \\
\hline$\Delta E_{Q} / \mathrm{mms}^{-1}$ & $0.80 \pm 0.05$ & 0 & $0 \pm 0.1$ & $2.20 \pm 0.05$ \\
\hline$\Gamma / \mathrm{mms}^{-1}$ & $0.35 \pm 0.05$ & 0 & $0.4 \pm 0.04$ & $0.4 \pm 0.05$ \\
\hline Area / \% & $82.8 \pm 2.0$ & 0 & $10.4 \pm 2.0$ & $6.9 \pm 3.0$ \\
\hline \multicolumn{5}{|l|}{$T=200 \mathrm{~K}$} \\
\hline$\delta / \mathrm{mms}^{-1}$ & $1.06 \pm 0.03$ & 0 & $0.55 \pm 0.02$ & $1.20 \pm 0.03$ \\
\hline$\Delta E_{Q} / \mathrm{mms}^{-1}$ & $1.15 \pm 0.03$ & 0 & $0 \pm 0.1$ & $2.35 \pm 0.03$ \\
\hline$\Gamma / \mathrm{mms}^{-1}$ & $0.40 \pm 0.03$ & 0 & $0.40 \pm 0.03$ & $0.40 \pm 0.02$ \\
\hline Area / \% & $84.3 \pm 2.0$ & 0 & $10.5 \pm 2.0$ & $5.2 \pm 2.0$ \\
\hline \multicolumn{5}{|l|}{$T=175 \mathrm{~K}$} \\
\hline$\delta / \mathrm{mms}^{-1}$ & $1.08 \pm 0.02$ & 0 & $0.55 \pm 0.02$ & $1.20 \pm 0.02$ \\
\hline$\Delta E_{Q} / \mathrm{mms}^{-1}$ & $1.30 \pm 0.03$ & 0 & $0 \pm 0.1$ & $3.00 \pm 0.03$ \\
\hline$\Gamma / \mathrm{mms}^{-1}$ & $0.40 \pm 0.02$ & 0 & $0.40 \pm 0.02$ & $0.4 \pm 0.03$ \\
\hline Area / \% & $83.8 \pm 2.0$ & 0 & $10.5 \pm 2.0$ & $5.8 \pm 2.0$ \\
\hline \multicolumn{5}{|l|}{$T=150 \mathrm{~K}$} \\
\hline$\delta / \mathrm{mms}^{-1}$ & $1.12 \pm 0.02$ & $0.35 \pm 0.02$ & $0.55 \pm 0.02$ & $1.20 \pm 0.02$ \\
\hline$\Delta E_{Q} / \mathrm{mms}^{-1}$ & $1.38 \pm 0.03$ & $0.50 \pm 0.04$ & $0 \pm 0.1$ & $3.00 \pm 0.02$ \\
\hline$\Gamma / \mathrm{mms}^{-1}$ & $0.40 \pm 0.03$ & $0.40 \pm 0.02$ & $0.40 \pm 0.02$ & $0.40 \pm 0.02$ \\
\hline Area / \% & $79.8 \pm 2.0$ & $6.8 \pm 2.0$ & $10.0 \pm 2.0$ & $3.4 \pm 1.0$ \\
\hline \multicolumn{5}{|l|}{$T=100 \mathrm{~K}$} \\
\hline$\delta / \mathrm{mms}^{-1}$ & $1.15 \pm 0.02$ & $0.39 \pm 0.02$ & $0.55 \pm 0.02$ & $1.30 \pm 0.02$ \\
\hline$\Delta E_{Q} / \mathrm{mms}^{-1}$ & $1.55 \pm 0.04$ & $0.55 \pm 0.04$ & $0 \pm 0.1$ & $3.00 \pm 0.02$ \\
\hline$\Gamma / \mathrm{mms}^{-1}$ & $0.42 \pm 0.03$ & $0.40 \pm 0.02$ & $0.40 \pm 0.02$ & $0.40 \pm 0.02$ \\
\hline Area / \% & $71.4 \pm 2.0$ & $15.6 \pm 2.0$ & $10.2 \pm 2.0$ & $2.9 \pm 1.0$ \\
\hline \multicolumn{5}{|l|}{$T=77 \mathrm{~K}$} \\
\hline$\delta / \mathrm{mms}^{-1}$ & $1.15 \pm 0.02$ & $0.42 \pm 0.02$ & $0.55 \pm 0.2$ & $1.30 \pm 0.02$ \\
\hline$\Delta E_{Q} / \mathrm{mms}^{-1}$ & $1.65 \pm 0.05$ & $0.55 \pm 0.03$ & $0 \pm 0.1$ & $3.00 \pm 0.03$ \\
\hline$\Gamma / \mathrm{mms}^{-1}$ & $0.42 \pm 0.02$ & $0.40 \pm 0.02$ & $0.40 \pm 0.02$ & $0.40 \pm 0.02$ \\
\hline Area / \% & $64.7 \pm 2.0$ & $21.6 \pm 2.0$ & $10.8 \pm 2.0$ & $2.9 \pm 1.0$ \\
\hline
\end{tabular}


Table S15 Temperature dependence of the high-spin fraction $\left(\gamma_{\mathrm{HS}}\right)$ of the iron(II) centers in the cubane assemblies, as measured by different techniques. These data are plotted in Figure 5 of the main article.

\begin{tabular}{lccc}
\hline$T / \mathrm{K}$ & \multicolumn{3}{c}{$\gamma_{\mathrm{HS}}[\mathrm{Fe}(\mathrm{II})]$} \\
\hline $\mathbf{1}$ or 1·6MeCN & Magnetic data $^{\mathrm{a}}$ & Crystallography $^{\mathrm{b}}$ & Mössbauer spectra \\
300 & $1.000 \pm 0.025$ & & \\
250 & $0.975 \pm 0.025$ & $0.94 \pm 0.06$ & $0.982 \pm 0.047^{\mathrm{c}}$ \\
200 & $0.914 \pm 0.025$ & $0.89 \pm 0.06$ & $1.000 \pm 0.047$ \\
175 & $0.875 \pm 0.025$ & & $0.994 \pm 0.047$ \\
150 & $0.832 \pm 0.025$ & $0.83 \pm 0.06$ & $0.947 \pm 0.046$ \\
125 & $0.786 \pm 0.025$ & & \\
100 & $0.743 \pm 0.025$ & $0.79 \pm 0.05$ & $0.847 \pm 0.044$ \\
77 & $0.696 \pm 0.025$ & & $0.768 \pm 0.042$ \\
& & & \\
$\mathbf{2} \cdot x \mathrm{MeCN} \cdot y \mathrm{Et}_{2} \mathrm{O}$ & & & \\
250 & $0.986 \pm 0.025$ & & \\
200 & $0.996 \pm 0.025$ & $0.996 \pm 0.007$ & \\
175 & $0.971 \pm 0.025$ & $0.970 \pm 0.007$ & \\
150 & $0.943 \pm 0.025$ & & \\
125 & $0.896 \pm 0.025$ & & \\
100 & $0.839 \pm 0.025$ & $0.822 \pm 0.007$ & \\
77 & $0.796 \pm 0.025$ & & \\
\hline
\end{tabular}

${ }^{\mathrm{a} C a l c u l a t e d}$ assuming $\chi_{\mathrm{M}} T=3.5 \mathrm{~cm}^{3} \mathrm{~mol}^{-1} \mathrm{~K}$ for high-spin iron(II), $\chi_{\mathrm{M}} T=0$ for low-spin iron(II) and $\chi_{\mathrm{M}} T=4.2 \mathrm{~cm}^{3} \mathrm{~mol}^{-1} \mathrm{~K}$ for high-spin iron(III). ${ }^{6}{ }^{\mathrm{b}}$ Calculated assuming $V_{\mathrm{Oh}}=12.9 \AA^{3}$ for high-spin iron(II), and $9.9 \AA^{3}$ for low-spin iron(II). ${ }^{2}$ Errors on these values are based on $3 \mathrm{x}$ the crystallographic esds. ${ }^{\mathrm{c}}$ Data point measured at $298 \mathrm{~K}$.

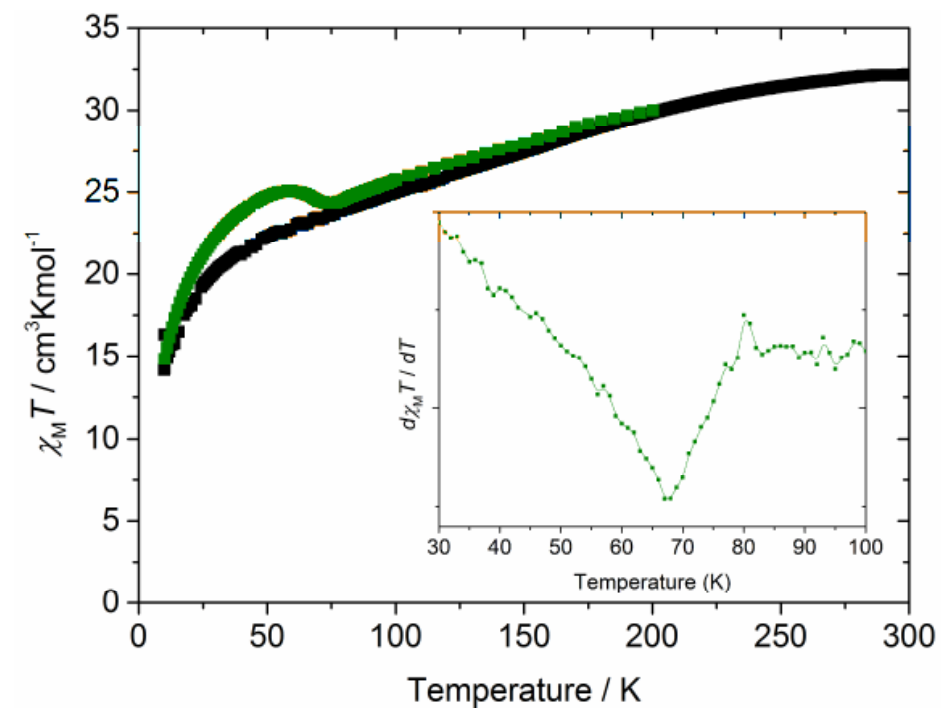

Figure S17 Solid state magnetic susceptibility data for $\mathbf{1}$ upon cooling in the dark (black), and rewarming following irradiation at $650 \mathrm{~nm}$ at $10 \mathrm{~K}$ (green). The inset shows the first derivative of the data from the irradiated sample, whose minimum corresponds to $T$ (LIESST) ${ }^{5}$

The black data points are the same as the aged sample in Figures S14. The LIESST measurement of $\mathbf{2}$ is shown in Figure 7 of the main article. 


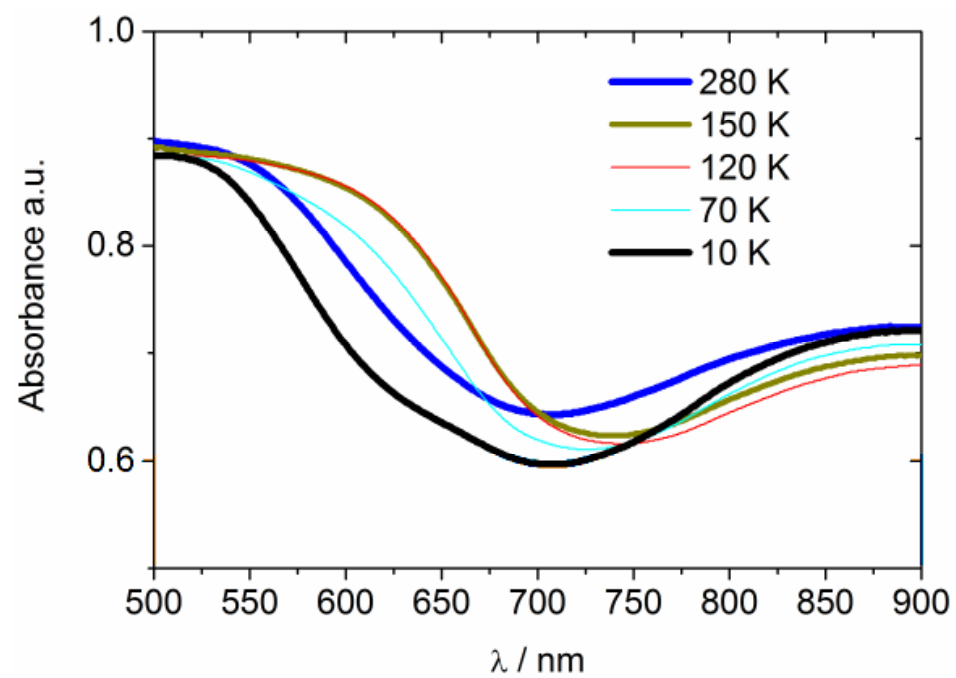

Figure S18 Diffuse reflectance UV/vis spectra of solid $\mathbf{1}$ at different temperatures.

The spectra at 280 and $10 \mathrm{~K}$ are from fully high-spin material, while the sample is in its (predominantly) low-spin form at the other temperatures. The high-spin nature of the sample at $10 \mathrm{~K}$ reflects its excitation by the incident white light, leading to light-induced spin-state trapping below $T$ (LIESST).

While the spectra show a vibronic temperature-dependence that is independent of SCO, the low-spin spectra are easily distinguishable in showing an increased absorbance between $550-750 \mathrm{~nm}$. That is consistent with the more intense MLCT absorptions in the visible region of the spectra, which are expected for low-spin iron(II) complexes with $N$-heterocyclic ligands. ${ }^{7}$

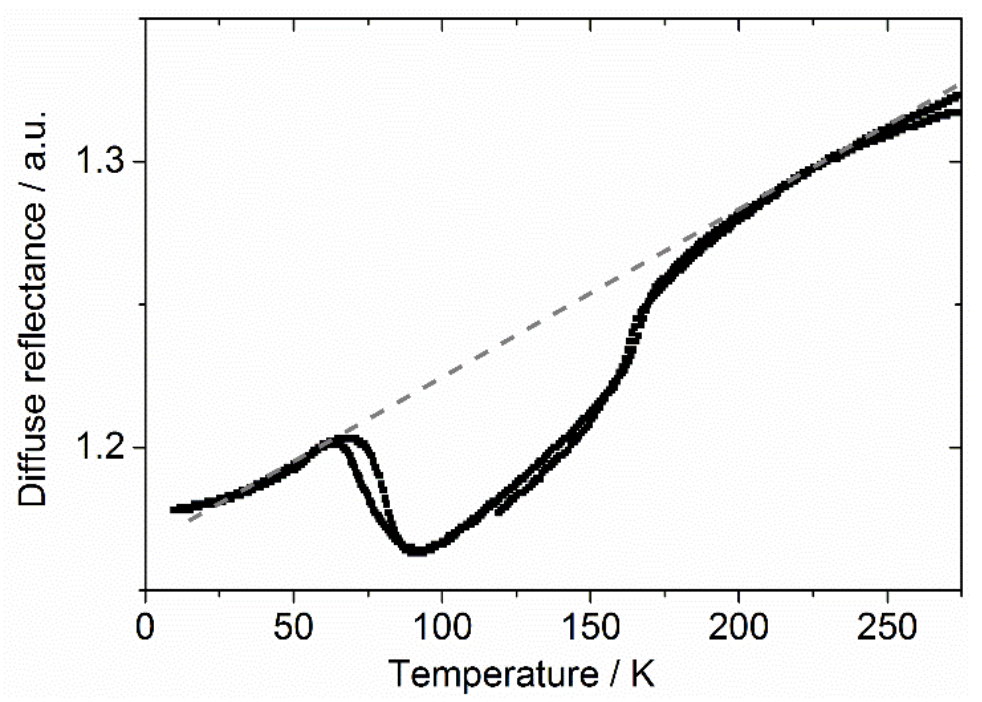

Figure S19 Temperature dependence of the total diffuse reflectance intensity from 1, on a $295 \rightarrow 10 \rightarrow 280 \mathrm{~K}$ temperature cycle. As a guide for the eye, the dotted line indicates the slope of the high-spin portion of the graph.

The additional drop in reflectance on cooling below $170 \mathrm{~K}$ is close to the onset of SCO in dried $\mathbf{1}$ by Mössbauer spectroscopy (Figure 5, main article), which converts some iron(II) centers to their more absorbing low-spin form. ${ }^{66}$ The increase in reflectance around $65 \mathrm{~K}$ reflects excitation by the white incident light of the low-spin fraction of the sample, which becomes trapped in its high-spin excited state at temperatures below $T$ (LIESST). 


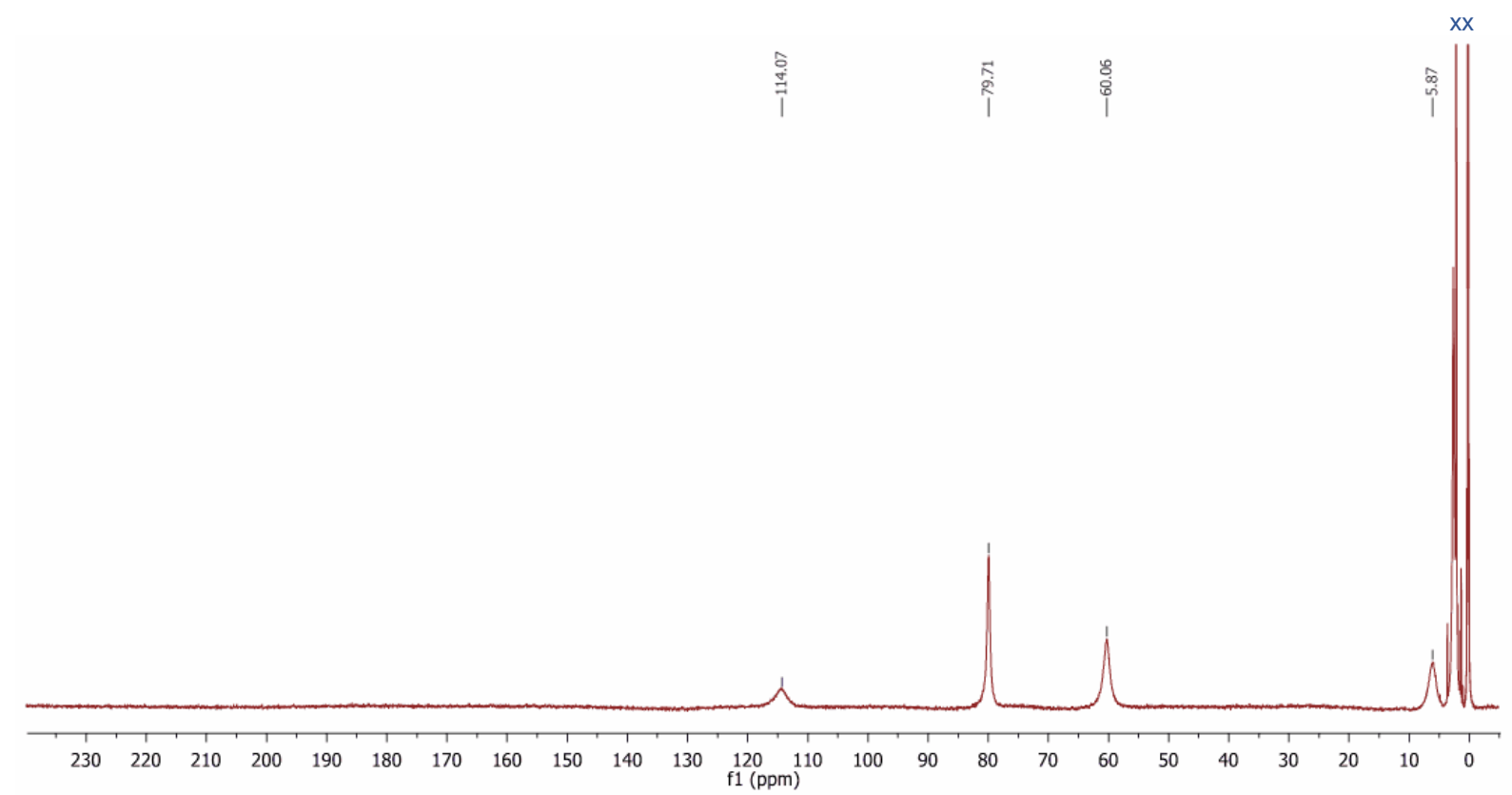

Figure S20 ${ }^{1} \mathrm{H}$ NMR spectrum of $1\left(400 \mathrm{MHz}, \mathrm{CD}_{3} \mathrm{CN}\right)$.

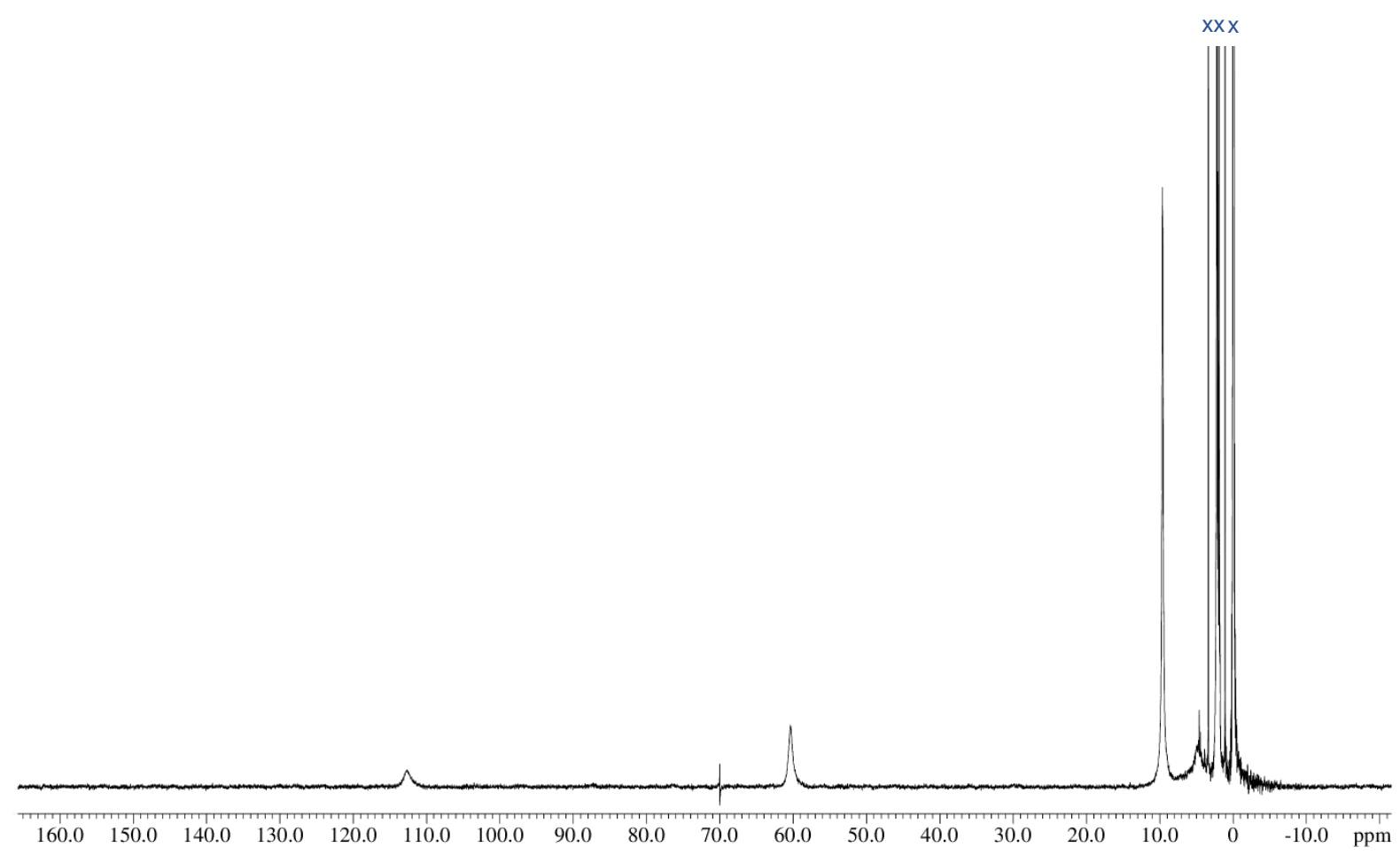

Figure S21 ${ }^{1} \mathrm{H}$ NMR spectrum of 2 (600 MHz, $\left.\mathrm{CD}_{3} \mathrm{CN} / \mathrm{TMS}\right)$. 


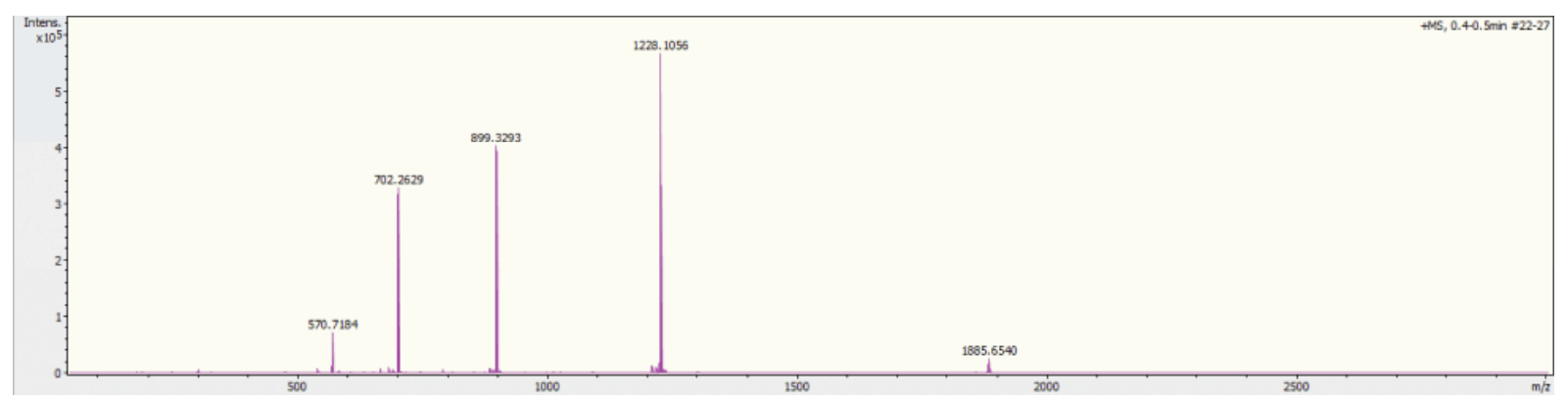

Figure S22 Electrospray mass spectrum of $\mathbf{1}$ from $\mathrm{MeCN}$ solution. This is an expansion of the same spectrum shown in Figure 7 of the main article.

Expansions and simulations of each peak are shown in Figure S23 below.
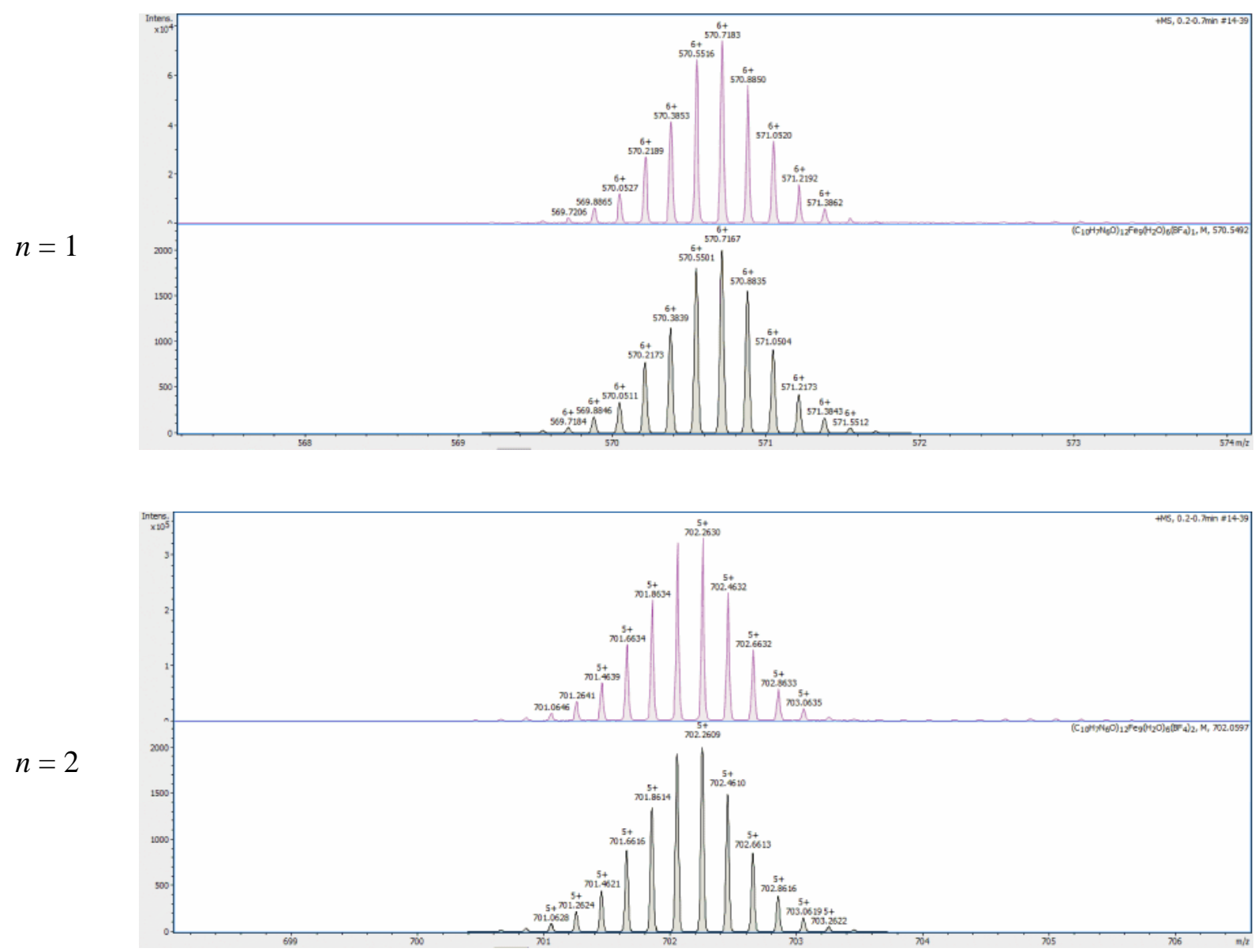

Figure S23 Measured (top) and simulated (bottom) ESMS mass peaks for the ions $\left[\left\{\mathrm{Fe}\left(\mathrm{OH}_{2}\right)_{6}\right\} \subset \mathrm{Fe}_{8}\left(\mu-L^{1}\right)_{12}\left(\mathrm{BF}_{4}\right)_{n}\right]^{(7-n)+}$. 

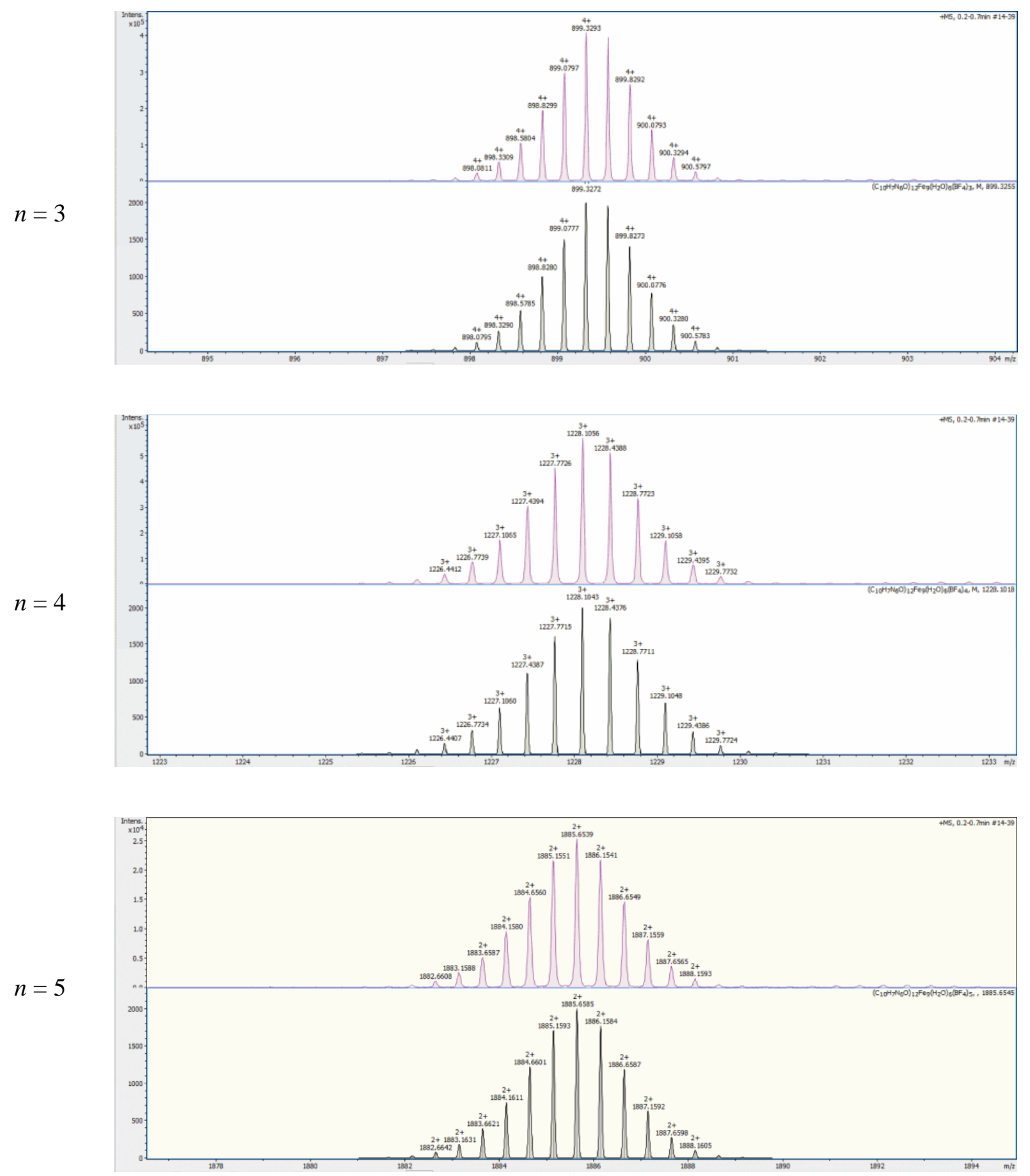

Figure S23 (continued). 


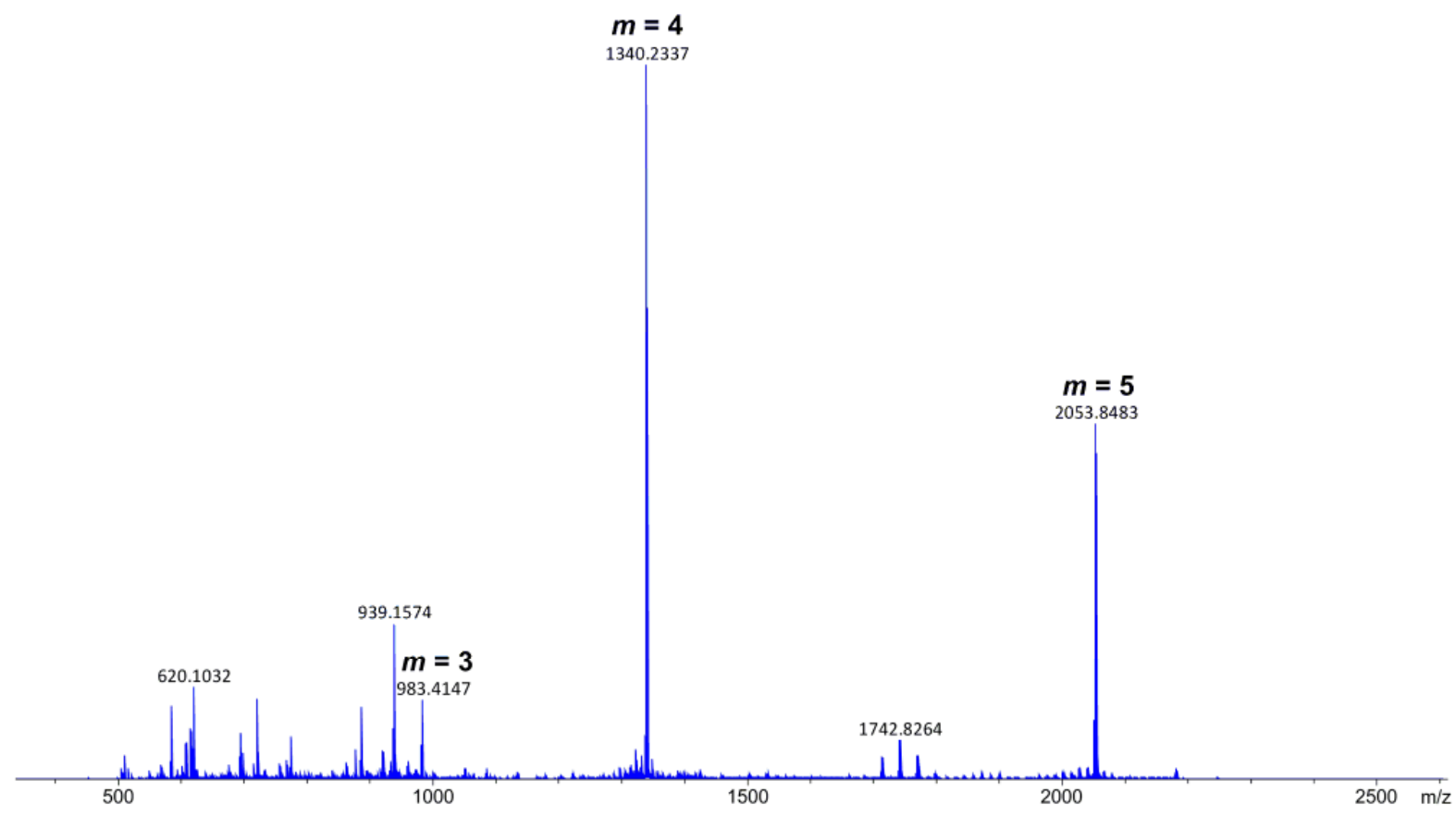

Figure S24 Electrospray mass spectrum of 2, showing the $\left[\left\{\mathrm{Fe}\left(\mathrm{OH}_{2}\right)_{6}\right\} \subset \mathrm{Fe}_{8}\left(\mu-L^{2}\right)_{12}\left(\mathrm{BF}_{4}\right)_{m}\right]^{(7-m)+}$ peak progression.

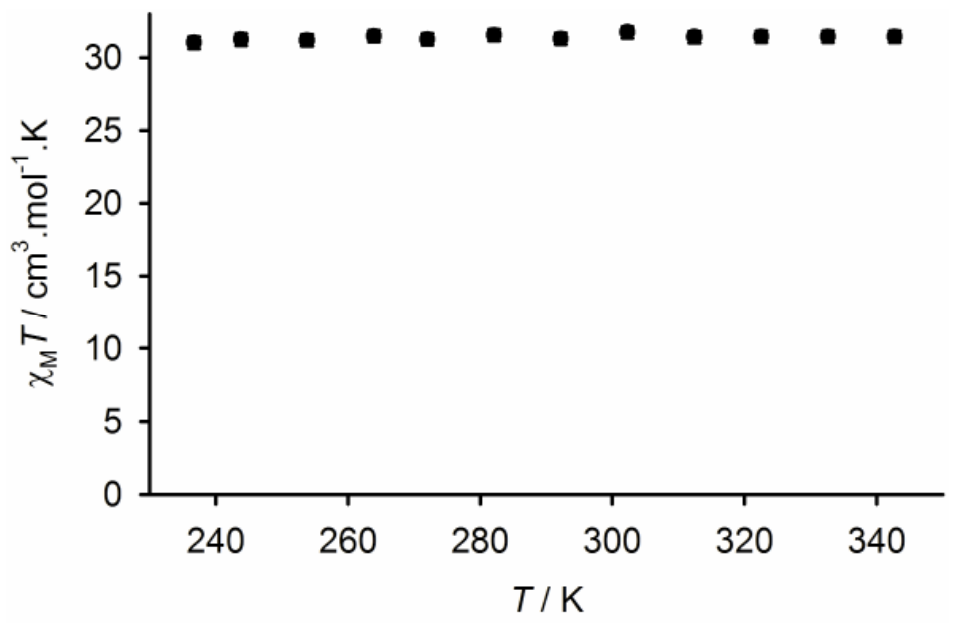

Figure S25 Variable temperature magnetic susceptibility data for 1 in $\mathrm{CD}_{3} \mathrm{CN}$ 


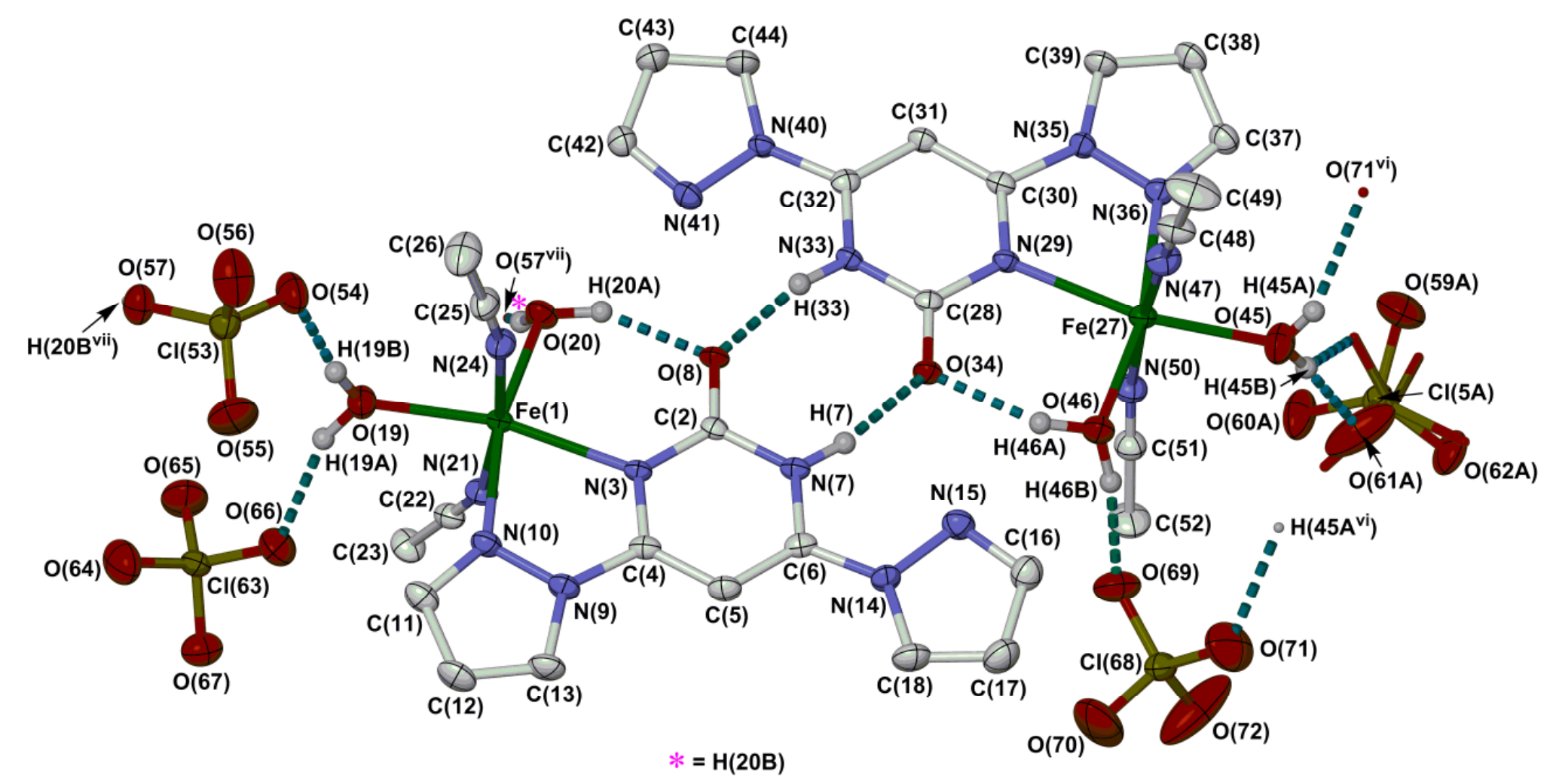

Figure S26 View of the asymmetric unit in $\left[\mathrm{Fe}\left(\mathrm{HL}^{1}\right)\left(\mathrm{OH}_{2}\right)_{2}(\mathrm{NCMe})_{2}\right]\left[\mathrm{ClO}_{4}\right]_{2}(3)$. The minor orientation of the disordered $\mathrm{ClO}_{4}{ }^{-}$ion is de-emphasised, and C-bound $\mathrm{H}$ atoms have been omitted for clarity. Atomic displacement ellipsoids are at the $50 \%$ probability level. Symmetry codes: (vi) $-x, 1-y, 1-z ;$ (vii) $2-x,-y, 2-z$.

Color code: $\mathrm{C}$, white; $\mathrm{H}$, pale gray; $\mathrm{Cl}$, dark yellow; $\mathrm{Fe}$, green; $\mathrm{N}$, blue; $\mathrm{O}$, red.

These supramolecular dimers associate, via hydrogen bonds to bridging perchlorate ions, into 1D chains along the crystallographic [2 $\overline{1} 1]$ vector (Table S8). 


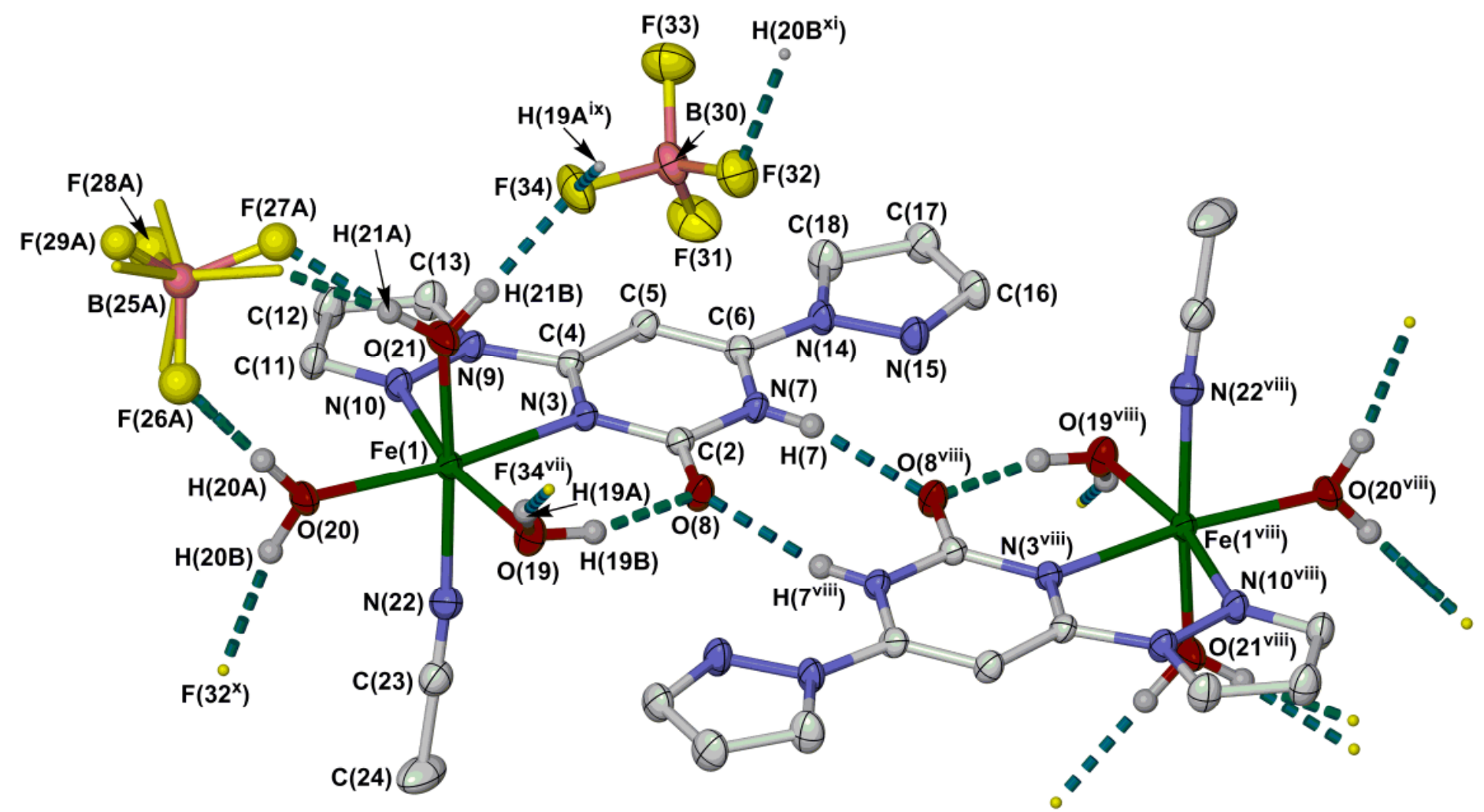

Figure S27 View of the asymmetric unit in $\left[\mathrm{Fe}\left(\mathrm{HL}^{1}\right)\left(\mathrm{OH}_{2}\right)_{3}(\mathrm{NCMe})\right]\left[\mathrm{BF}_{4}\right]_{2}(4)$. One orientation of the disordered $\mathrm{BF}_{4}^{-}$ion is de-emphasised, and $\mathrm{C}-\mathrm{bound} \mathrm{H}^{-}$atoms have been omitted for clarity. Atomic displacement ellipsoids are at the $50 \%$ probability level. Symmetry codes: (viii) $1-x, 1-y, 2-z$; (ix) $1-x, 2-y, 2-z$;

(x) $-1+x, y, z$; (xi) $1+x, y, z$.

Color code: C, white; H, pale gray; B, pink; F, yellow; Fe, green; N, blue; O, red. 
Table S16 Selected bond lengths and angles in the crystal structure of $\mathbf{3}\left(\AA{ }^{\circ}{ }^{\circ}\right)$. See Fig. S26 for the atom numbering scheme employed.

\begin{tabular}{llll}
\hline $\mathrm{Fe}(1)-\mathrm{N}(3)$ & $2.240(2)$ & $\mathrm{Fe}(27)-\mathrm{N}(29)$ & $2.239(2)$ \\
$\mathrm{Fe}(1)-\mathrm{N}(10)$ & $2.146(2)$ & $\mathrm{Fe}(27)-\mathrm{N}(36)$ & $2.134(2)$ \\
$\mathrm{Fe}(1)-\mathrm{O}(19)$ & $2.092(2)$ & $\mathrm{Fe}(27)-\mathrm{O}(45)$ & $2.100(2)$ \\
$\mathrm{Fe}(1)-\mathrm{O}(20)$ & $2.082(2)$ & $\mathrm{Fe}(27)-\mathrm{O}(46)$ & $2.088(2)$ \\
$\mathrm{Fe}(1)-\mathrm{N}(21)$ & $2.151(3)$ & $\mathrm{Fe}(27)-\mathrm{N}(47)$ & $2.140(3)$ \\
$\mathrm{Fe}(1)-\mathrm{N}(24)$ & $2.132(3)$ & $\mathrm{Fe}(27)-\mathrm{N}(50)$ & $2.139(3)$ \\
& & & \\
$\mathrm{N}(3)-\mathrm{Fe}(1)-\mathrm{N}(10)$ & $73.38(9)$ & $\mathrm{N}(29)-\mathrm{Fe}(27)-\mathrm{N}(36)$ & $74.10(9)$ \\
$\mathrm{N}(3)-\mathrm{Fe}(1)-\mathrm{O}(19)$ & $165.46(9)$ & $\mathrm{N}(29)-\mathrm{Fe}(27)-\mathrm{O}(45)$ & $165.82(10)$ \\
$\mathrm{N}(3)-\mathrm{Fe}(1)-\mathrm{O}(20)$ & $94.16(9)$ & $\mathrm{N}(29)-\mathrm{Fe}(27)-\mathrm{O}(46)$ & $93.37(9)$ \\
$\mathrm{N}(3)-\mathrm{Fe}(1)-\mathrm{N}(21)$ & $95.80(9)$ & $\mathrm{N}(29)-\mathrm{Fe}(27)-\mathrm{N}(47)$ & $93.90(10)$ \\
$\mathrm{N}(3)-\mathrm{Fe}(1)-\mathrm{N}(24)$ & $91.82(9)$ & $\mathrm{N}(29)-\mathrm{Fe}(27)-\mathrm{N}(50)$ & $91.59(10)$ \\
$\mathrm{N}(10)-\mathrm{Fe}(1)-\mathrm{O}(19)$ & $92.17(10)$ & $\mathrm{N}(36)-\mathrm{Fe}(27)-\mathrm{O}(45)$ & $92.04(10)$ \\
$\mathrm{N}(10)-\mathrm{Fe}(1)-\mathrm{O}(20)$ & $166.67(10)$ & $\mathrm{N}(36)-\mathrm{Fe}(27)-\mathrm{O}(46)$ & $167.36(10)$ \\
$\mathrm{N}(10)-\mathrm{Fe}(1)-\mathrm{N}(21)$ & $89.42(10)$ & $\mathrm{N}(36)-\mathrm{Fe}(27)-\mathrm{N}(47)$ & $91.62(10)$ \\
$\mathrm{N}(10)-\mathrm{Fe}(1)-\mathrm{N}(24)$ & $93.21(10)$ & $\mathrm{N}(36)-\mathrm{Fe}(27)-\mathrm{N}(50)$ & $94.42(10)$ \\
$\mathrm{O}(19)-\mathrm{Fe}(1)-\mathrm{O}(20)$ & $100.37(9)$ & $\mathrm{O}(45)-\mathrm{Fe}(27)-\mathrm{O}(46)$ & $100.56(10)$ \\
$\mathrm{O}(19)-\mathrm{Fe}(1)-\mathrm{N}(21)$ & $85.44(10)$ & $\mathrm{O}(45)-\mathrm{Fe}(27)-\mathrm{N}(47)$ & $89.39(11)$ \\
$\mathrm{O}(19)-\mathrm{Fe}(1)-\mathrm{N}(24)$ & $87.30(10)$ & $\mathrm{O}(45)-\mathrm{Fe}(27)-\mathrm{N}(50)$ & $86.42(10)$ \\
$\mathrm{O}(20)-\mathrm{Fe}(1)-\mathrm{N}(21)$ & $87.11(9)$ & $\mathrm{O}(46)-\mathrm{Fe}(27)-\mathrm{N}(47)$ & $87.44(10)$ \\
$\mathrm{O}(20)-\mathrm{Fe}(1)-\mathrm{N}(24)$ & $91.88(10)$ & $\mathrm{O}(46)-\mathrm{Fe}(27)-\mathrm{N}(50)$ & $87.53(10)$ \\
$\mathrm{N}(21)-\mathrm{Fe}(1)-\mathrm{N}(24)$ & $172.36(10)$ & $\mathrm{N}(47)-\mathrm{Fe}(27)-\mathrm{N}(50)$ & $172.76(11)$ \\
\hline
\end{tabular}

Table S17 Hydrogen bond parameters for the crystal structures of $3\left(\AA,{ }^{\circ}\right)$. See Fig. S26 for the atom numbering scheme. Symmetry codes: (vi) $-x, 1-y, 1-z$; (vii) $2-x,-y, 2-z$.

\begin{tabular}{lllll}
\hline & $\mathrm{D}-\mathrm{H}$ & $\mathrm{H} \ldots \mathrm{A}$ & $\mathrm{D} \ldots \mathrm{A}$ & $\mathrm{D}-\mathrm{H} \ldots \mathrm{A}$ \\
\hline $\mathrm{N}(7)-\mathrm{H}(7) \ldots \mathrm{O}(34)$ & 0.88 & 2.00 & $2.846(3)$ & 159.5 \\
$\mathrm{O}(19)-\mathrm{H}(19 \mathrm{~A}) \ldots \mathrm{O}(66)$ & $0.892(18)$ & $1.98(2)$ & $2.831(4)$ & $159(4)$ \\
$\mathrm{O}(19)-\mathrm{H}(19 \mathrm{~B}) \ldots \mathrm{O}(54)$ & $0.885(18)$ & $1.944(18)$ & $2.822(3)$ & $171(4)$ \\
$\mathrm{O}(20)-\mathrm{H}(20 \mathrm{~A}) \ldots \mathrm{O}(8)$ & $0.896(18)$ & $1.92(2)$ & $2.733(3)$ & $151(4)$ \\
$\mathrm{O}(20)-\mathrm{H}(20 \mathrm{~B}) \ldots \mathrm{O}\left(57^{\mathrm{vii}}\right)$ & $0.879(18)$ & $1.96(2)$ & $2.809(3)$ & $162(3)$ \\
$\mathrm{N}(33)-\mathrm{H}(33) \ldots \mathrm{O}(8)$ & 0.88 & 2.05 & $2.888(3)$ & 158.9 \\
$\mathrm{O}(45)-\mathrm{H}(45 \mathrm{~A}) \ldots \mathrm{O}\left(71^{\mathrm{vi}}\right)$ & $0.871(18)$ & $2.08(3)$ & $2.891(4)$ & $154(5)$ \\
$\mathrm{O}(45)-\mathrm{H}(45 \mathrm{~B}) \ldots \mathrm{O}(61 \mathrm{~A} / \mathrm{B})$ & $0.880(18)$ & $1.867(19) / 2.04(3)$ & $2.742(6) / 2.786(9)$ & $174(5) / 142(4)$ \\
$\mathrm{O}(46)-\mathrm{H}(46 \mathrm{~A}) \ldots \mathrm{O}(34)$ & $0.887(18)$ & $1.89(2)$ & $2.722(3)$ & $155(4)$ \\
$\mathrm{O}(46)-\mathrm{H}(46 \mathrm{~B}) \ldots \mathrm{O}(69)$ & $0.889(18)$ & $1.93(2)$ & $2.805(4)$ & $168(4)$ \\
\hline
\end{tabular}


Table S18 Selected bond lengths and angles in the crystal structure of $4\left(\AA,^{\circ}\right)$. See Fig. S27 for the atom numbering scheme employed.

\begin{tabular}{llll}
\hline $\mathrm{Fe}(1)-\mathrm{N}(3)$ & $2.232(3)$ & $\mathrm{Fe}(1)-\mathrm{O}(20)$ & $2.092(2)$ \\
$\mathrm{Fe}(1)-\mathrm{N}(10)$ & $2.145(3)$ & $\mathrm{Fe}(1)-\mathrm{O}(21)$ & $2.127(3)$ \\
$\mathrm{Fe}(1)-\mathrm{O}(19)$ & $2.080(3)$ & $\mathrm{Fe}(1)-\mathrm{N}(22)$ & $2.147(3)$ \\
& & & \\
$\mathrm{N}(3)-\mathrm{Fe}(1)-\mathrm{N}(10)$ & $73.48(10$ & $\mathrm{N}(10)-\mathrm{Fe}(1)-\mathrm{N}(22)$ & $92.09(12)$ \\
$\mathrm{N}(3)-\mathrm{Fe}(1)-\mathrm{O}(19)$ & $93.41(10)$ & $\mathrm{O}(19)-\mathrm{Fe}(1)-\mathrm{O}(20)$ & $100.55(10)$ \\
$\mathrm{N}(3)-\mathrm{Fe}(1)-\mathrm{O}(20)$ & $165.88(11)$ & $\mathrm{O}(19)-\mathrm{Fe}(1)-\mathrm{O}(21)$ & $87.83(12)$ \\
$\mathrm{N}(3)-\mathrm{Fe}(1)-\mathrm{O}(21)$ & $94.67(10)$ & $\mathrm{O}(19)-\mathrm{Fe}(1)-\mathrm{N}(22)$ & $88.45(11)$ \\
$\mathrm{N}(3)-\mathrm{Fe}(1)-\mathrm{N}(22)$ & $91.77(10)$ & $\mathrm{O}(20)-\mathrm{Fe}(1)-\mathrm{O}(21)$ & $88.00(10)$ \\
$\mathrm{N}(10)-\mathrm{Fe}(1)-\mathrm{O}(19)$ & $166.89(10)$ & $\mathrm{O}(20)-\mathrm{Fe}(1)-\mathrm{N}(22)$ & $86.56(11)$ \\
$\mathrm{N}(10)-\mathrm{Fe}(1)-\mathrm{O}(20)$ & $92.55(11)$ & $\mathrm{O}(21)-\mathrm{Fe}(1)-\mathrm{N}(22)$ & $172.75(11)$ \\
$\mathrm{N}(10)-\mathrm{Fe}(1)-\mathrm{O}(21)$ & $92.94(12)$ & & \\
\hline
\end{tabular}

Table S19 Hydrogen bond parameters for the crystal structures of $4\left(\AA,{ }^{\circ}\right)$. See Fig. S27 for the atom numbering scheme. Symmetry codes: (viii) $1-x, 1-y, 2-z$; (ix) $1-x, 2-y, 2-z$; (x) $-1+x, y, z$.

\begin{tabular}{lllll}
\hline & $\mathrm{D}-\mathrm{H}$ & $\mathrm{H} \ldots \mathrm{A}$ & $\mathrm{D} \ldots \mathrm{A}$ & $\mathrm{D}-\mathrm{H} \ldots \mathrm{A}$ \\
\hline $\mathrm{N}(7)-\mathrm{H}(7) \ldots \mathrm{O}\left(8^{\text {viii }}\right)$ & $0.83(5)$ & $2.05(5)$ & $2.867(4)$ & $167(4)$ \\
$\mathrm{O}(19)-\mathrm{H}(19 \mathrm{~A}) \ldots \mathrm{F}\left(34^{\mathrm{ix}}\right)$ & $0.89(2)$ & $2.07(2)$ & $2.928(4)$ & $162(5)$ \\
$\mathrm{O}(19)-\mathrm{H}(19 \mathrm{~B}) \ldots \mathrm{O}(8)$ & $0.894(19)$ & $1.84(3)$ & $2.684(3)$ & $156(5)$ \\
$\mathrm{O}(20)-\mathrm{H}(20 \mathrm{~A}) \ldots \mathrm{F}(26 \mathrm{~A}) / \mathrm{F}(26 \mathrm{~B})$ & $0.87(2)$ & $1.81(2) / 1.85(2)$ & $2.683(5) / 2.699(7)$ & $175(5) / 163(5)$ \\
$\mathrm{O}(20)-\mathrm{H}(20 \mathrm{~B}) \ldots \mathrm{F}\left(32^{\mathrm{x}}\right)$ & $0.88(2)$ & $1.98(2)$ & $2.829(4)$ & $163(5)$ \\
$\mathrm{O}(21)-\mathrm{H}(21 \mathrm{~A}) \ldots \mathrm{F}(27 \mathrm{~A}) / \mathrm{F}(27 \mathrm{~B})$ & $0.88(2)$ & $1.86(2) / 1.83(2)$ & $2.735(5) / 2.706(6)$ & $173(5) / 171(5)$ \\
$\mathrm{O}(21)-\mathrm{H}(21 \mathrm{~B}) \ldots \mathrm{F}(34)$ & $0.88(2)$ & $1.85(2)$ & $2.716(4)$ & $171(5)$ \\
\hline
\end{tabular}




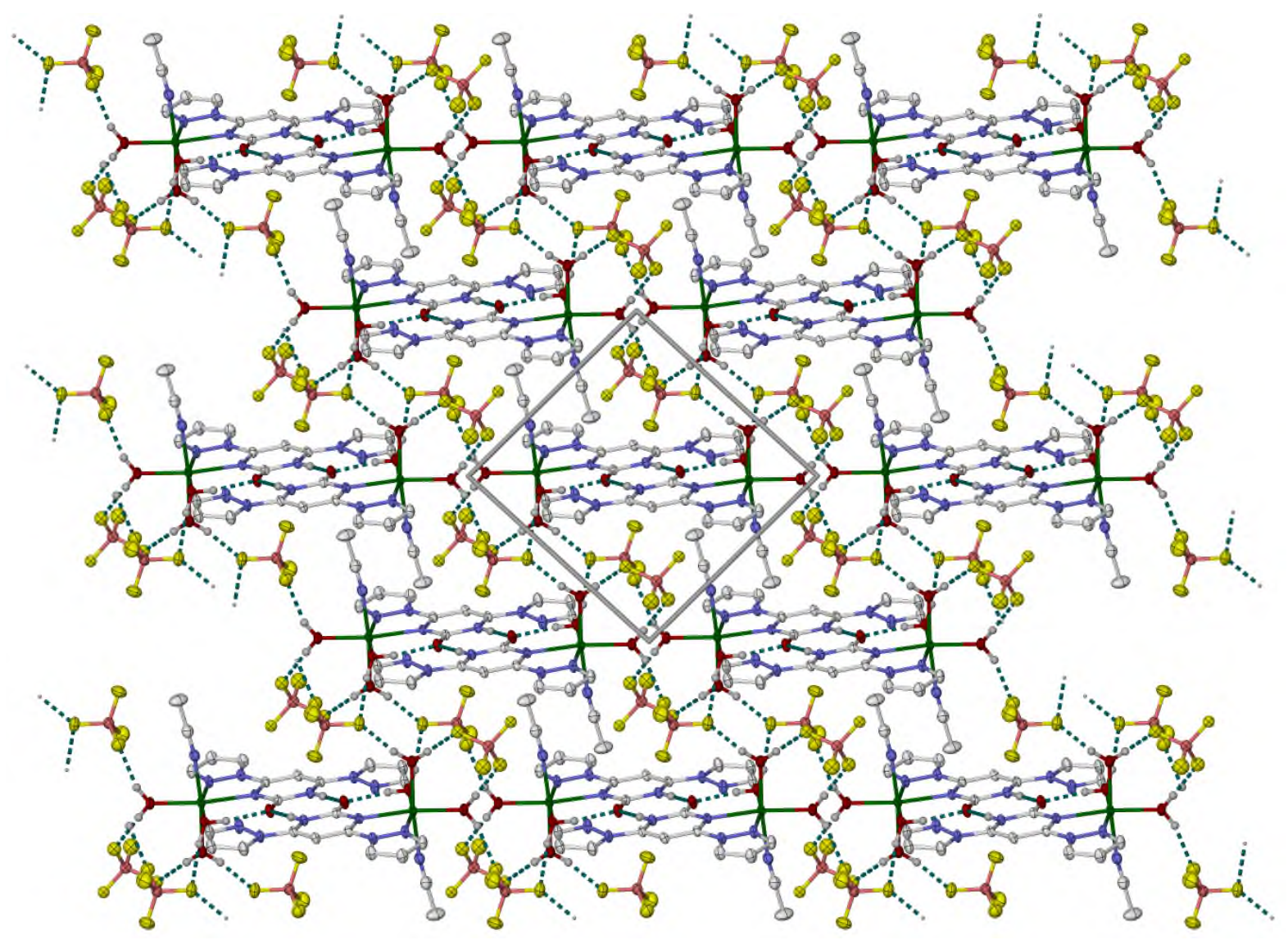

Figure S28 Packing diagram of $\mathbf{4}$, showing the association of the hydrogen-bonded dimers into sheets parallel to (001). Only one orientation of the disordered $\mathrm{BF}_{4}^{-}$ion is shown, and $\mathrm{C}$-bound $\mathrm{H}$ atoms have been omitted for clarity. Atomic displacement ellipsoids are at the $50 \%$ probability level. The view is parallel to the (001) crystal vector.

Color code: C, white; H, pale gray; B, pink; F, yellow; Fe, green; N, blue; O, red.

The hydrogen bonding topology in the bilayers is $\left(4^{2} 6^{2}\right)\left(4^{2} 6\right)$ in the short Schläfli notation, ${ }^{8}$ with the complex cation and bridging $\mathrm{BF}_{4}^{-}$ion both acting as three-connected nodes. 


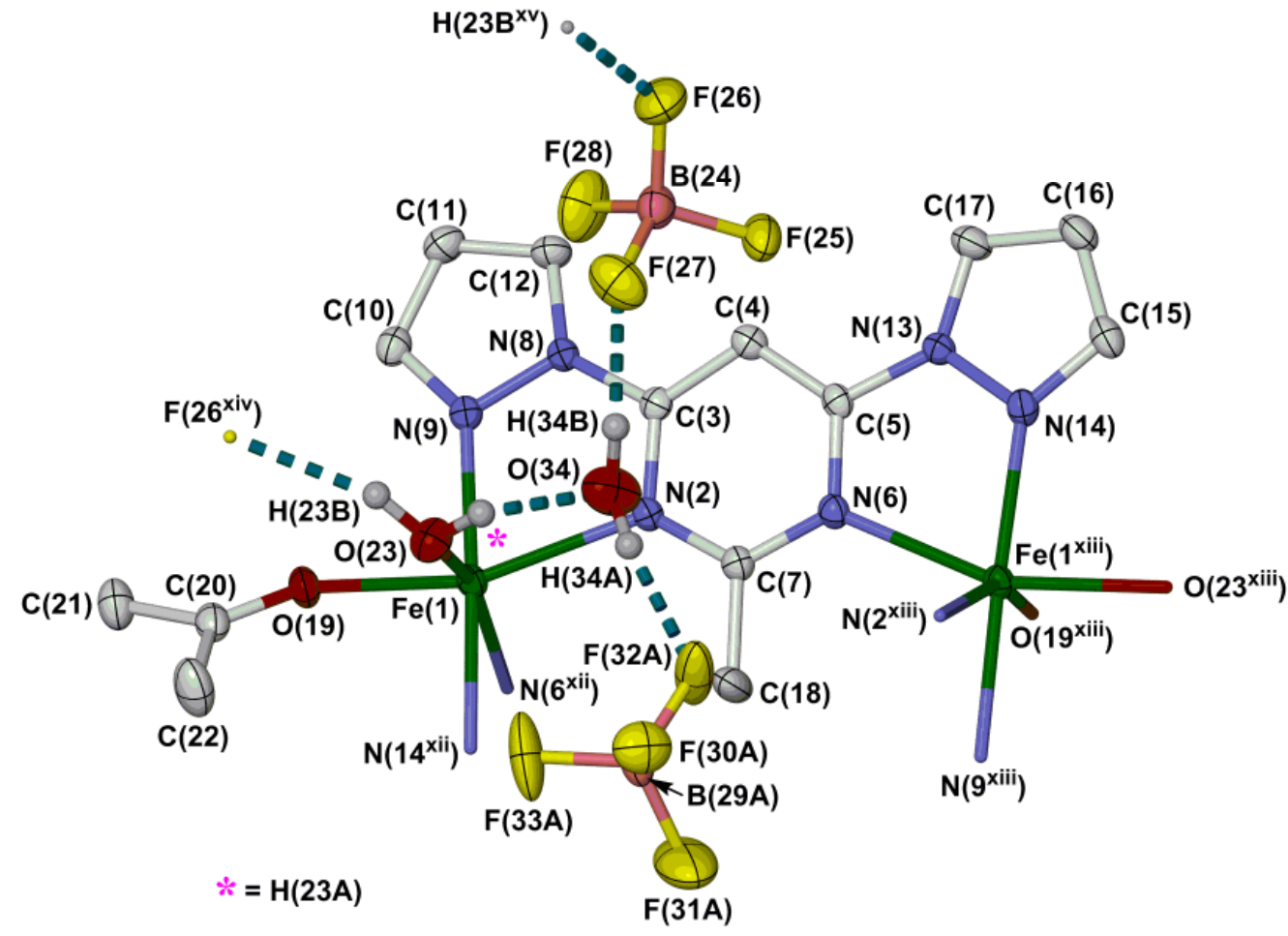

Figure S29 View of the asymmetric unit in $\left[\mathrm{Fe}\left(\mu-L^{3}\right)\left(\mathrm{OCMe}_{2}\right)\left(\mathrm{OH}_{2}\right)\right]\left[\mathrm{BF}_{4}\right]_{2} \cdot \mathrm{H}_{2} \mathrm{O}\left(\mathbf{5} \cdot \mathrm{H}_{2} \mathrm{O}\right)$. Only the major orientation of the disordered $\mathrm{BF}_{4}^{-}$ion $\mathrm{B}(29)-\mathrm{F}(33)$ is shown, and $\mathrm{C}$-bound $\mathrm{H}$ atoms have been omitted for clarity. Atomic displacement ellipsoids are at the $50 \%$ probability level. Symmetry codes:

(xii) ${ }^{3} / 2-x,-1 / 2+y,{ }^{3} / 2-z$; (xiii) ${ }^{3} / 2-x,{ }^{1} / 2+y,{ }^{3} / 2-z$; (xiv) $1 / 2-x,-1 / 2+y,{ }^{3} / 2-z$; (xv) $1 / 2-x, 1 / 2+y,{ }^{3} / 2-z$.

Color code: $\mathrm{C}$, white; $\mathrm{B}$, pink; F, yellow; Fe, green; N, blue; O, red.

Table S20 Selected bond lengths and angles in the crystal structure of $\mathbf{5} \cdot \mathrm{H}_{2} \mathrm{O}\left(\AA{ }^{\circ},{ }^{o}\right)$. See Fig. S29 for the atom numbering scheme. Symmetry code: (xii) $3 / 2-x,-1 / 2+y, 3 / 2-z$.

\begin{tabular}{llll}
$\mathrm{Fe}(1)-\mathrm{N}(2)$ & $2.2804(17)$ & $\mathrm{Fe}(1)-\mathrm{N}\left(14^{\mathrm{xii}}\right)$ & $2.1224(19)$ \\
$\mathrm{Fe}(1)-\mathrm{N}\left(6^{\mathrm{xii}}\right)$ & $2.2935(18)$ & $\mathrm{Fe}(1)-\mathrm{O}(19)$ & $2.1247(15)$ \\
$\mathrm{Fe}(1)-\mathrm{N}(9)$ & $2.1130(18)$ & $\mathrm{Fe}(1)-\mathrm{O}(23)$ & $2.0995(16)$ \\
& & & \\
$\mathrm{N}(2)-\mathrm{Fe}(1)-\mathrm{N}\left(6^{\mathrm{xii}}\right)$ & $97.02(6)$ & $\mathrm{N}\left(6^{\mathrm{x}}\right)-\mathrm{Fe}(1)-\mathrm{O}(23)$ & $159.32(7)$ \\
$\mathrm{N}(2)-\mathrm{Fe}(1)-\mathrm{N}(9)$ & $74.33(7)$ & $\mathrm{N}(9)-\mathrm{Fe}(1)-\mathrm{N}\left(14^{\mathrm{xii}}\right)$ & $177.41(7)$ \\
$\mathrm{N}(2)-\mathrm{Fe}(1)-\mathrm{N}\left(14^{\mathrm{xii}}\right)$ & $107.69(7)$ & $\mathrm{N}(9)-\mathrm{Fe}(1)-\mathrm{O}(19)$ & $84.67(7)$ \\
$\mathrm{N}(2)-\mathrm{Fe}(1)-\mathrm{O}(19)$ & $158.33(6)$ & $\mathrm{N}(9)-\mathrm{Fe}(1)-\mathrm{O}(23)$ & $92.81(7)$ \\
$\mathrm{N}(2)-\mathrm{Fe}(1)-\mathrm{O}(23)$ & $91.12(7)$ & $\mathrm{N}\left(14^{\mathrm{xii}}\right)-\mathrm{Fe}(1)-\mathrm{O}(19)$ & $93.46(7)$ \\
$\mathrm{N}\left(6^{\mathrm{xii}}\right)-\mathrm{Fe}(1)-\mathrm{N}(9)$ & $107.66(7)$ & $\mathrm{N}\left(14^{\mathrm{xii}}\right)-\mathrm{Fe}(1)-\mathrm{O}(23)$ & $85.57(7)$ \\
$\mathrm{N}\left(6^{\mathrm{xii}}\right)-\mathrm{Fe}(1)-\mathrm{N}\left(14^{\mathrm{xii}}\right)$ & $73.86(7)$ & $\mathrm{O}(19)-\mathrm{Fe}(1)-\mathrm{O}(23)$ & $95.23(7)$ \\
$\mathrm{N}\left(6^{\mathrm{xii}}\right)-\mathrm{Fe}(1)-\mathrm{O}(19)$ & $84.15(6)$ & & \\
\hline
\end{tabular}


Table S21 Hydrogen bond parameters for the crystal structure of $5 \cdot \mathrm{H}_{2} \mathrm{O}\left(\AA{ }^{\circ},{ }^{\circ}\right)$. See Fig. S29 for the atom numbering scheme. Symmetry code: (xiv) $1 / 2-x,-1 / 2+y, 3 / 2-z$.

\begin{tabular}{lllll}
\hline & $\mathrm{D}-\mathrm{H}$ & $\mathrm{H} \ldots \mathrm{A}$ & $\mathrm{D} \ldots \mathrm{A}$ & $\mathrm{D}-\mathrm{H} \ldots \mathrm{A}$ \\
\hline $\mathrm{O}(23)-\mathrm{H}(23 \mathrm{~A}) \ldots \mathrm{O}(34)$ & $0.876(18)$ & $1.79(2)$ & $2.636(3)$ & $161(3)$ \\
$\mathrm{O}(23)-\mathrm{H}(23 \mathrm{~B}) \ldots \mathrm{F}\left(26^{\text {xiv }}\right)$ & $0.860(18)$ & $1.90(2)$ & $2.731(2)$ & $162(3)$ \\
$\mathrm{O}(34)-\mathrm{H}(34 \mathrm{~A}) \ldots \mathrm{F}(27)$ & $0.905(19)$ & $1.86(2)$ & $2.758(3)$ & $169(4)$ \\
$\mathrm{O}(34)-\mathrm{H}(34 \mathrm{~B}) \ldots \mathrm{F}(32 \mathrm{~A}) / \mathrm{F}(33 \mathrm{~B})$ & $0.895(19)$ & $1.85(2) / 2.05(3)$ & $2.713(3) / 2.776(9)$ & $160(4) / 137(3)$ \\
\hline
\end{tabular}

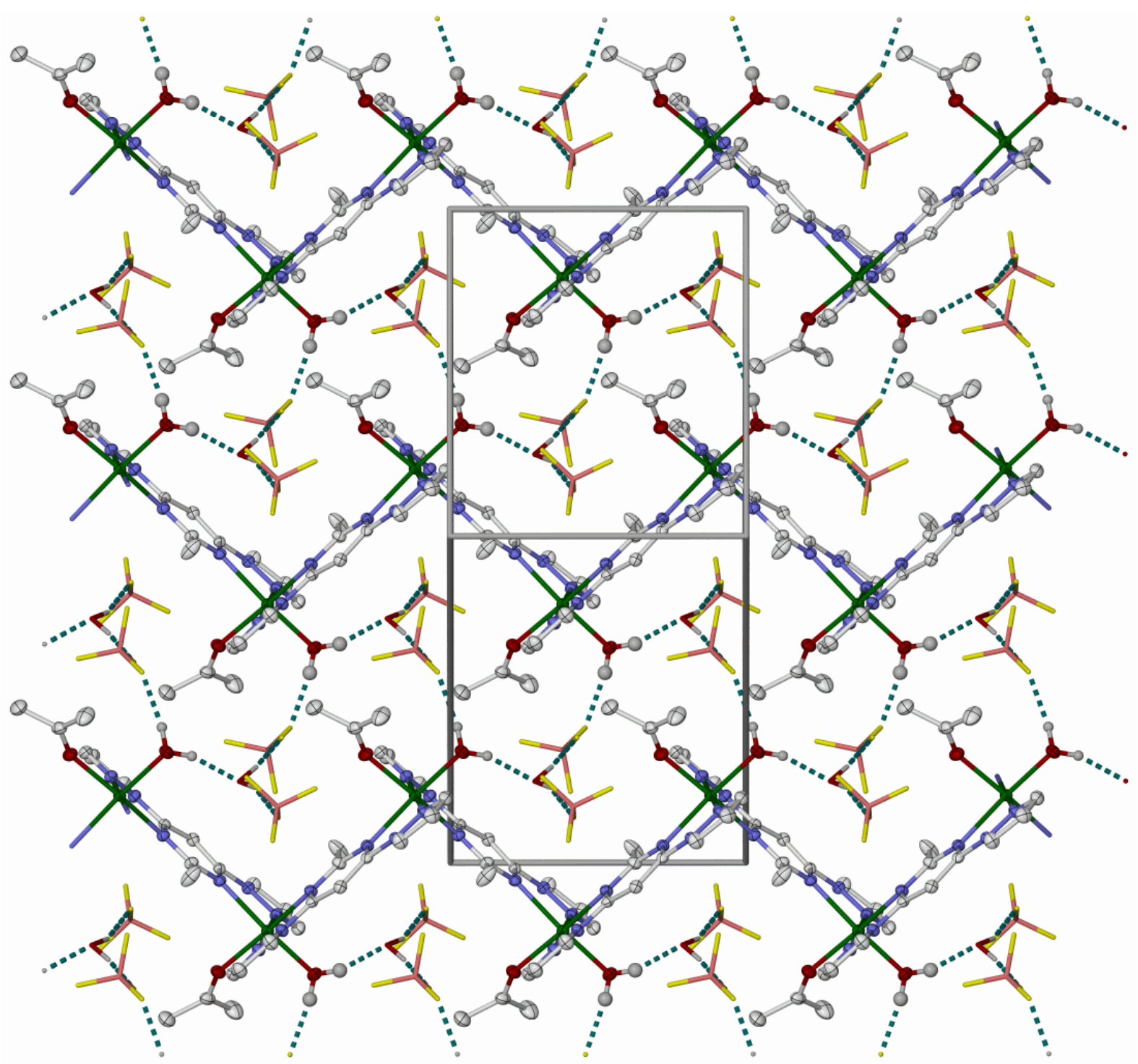

Figure S30 Packing diagram of $5 \cdot \mathrm{H}_{2} \mathrm{O}$, showing the association of coordination polymer chains into hydrogen bonded sheets. Displacement ellipsoids are at the $50 \%$ probability level except for the $\mathrm{BF}_{4}{ }^{-}$ions and lattice water which are de-emphasized for clarity. Only one orientation of the disordered anion environment is shown. The view is parallel to the [101] crystal vector, with $b$ horizontal.

Color code: $\mathrm{C}$, white; $\mathrm{B}$, pink; F, yellow; Fe, green; N, blue; O, red. 

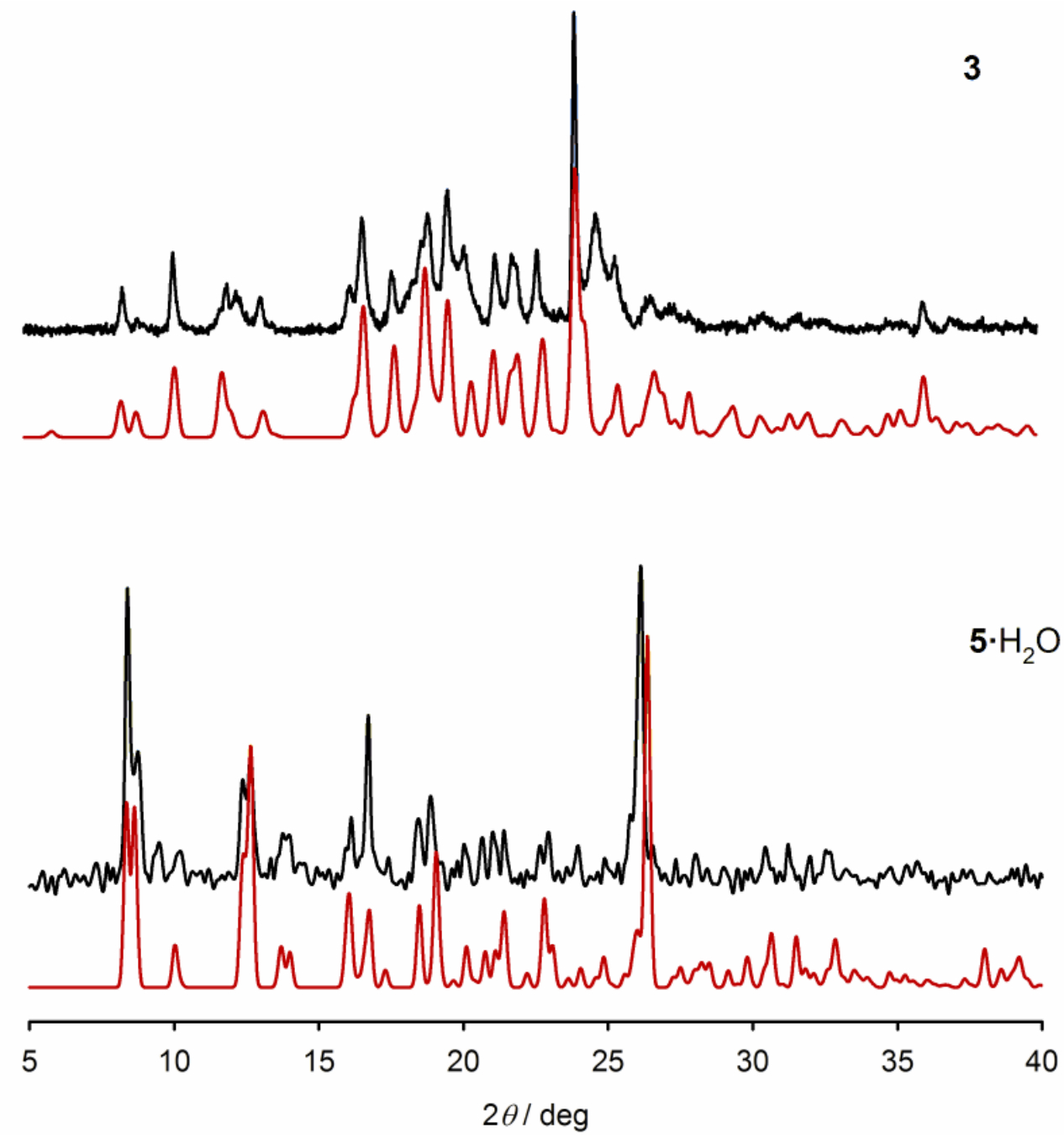

Figure S31 Black: room-temperature X-ray powder diffraction data for air-dried samples of $\mathbf{3}$ (top) and $5 \cdot \mathrm{H}_{2} \mathrm{O}$ (bottom). Red: simulated powder patterns of the same compounds from their low temperature crystal structures.

Agreement between these measured and simulated data is generally good, although $\mathbf{5} \cdot \mathrm{H}_{2} \mathrm{O}$ exhibits an additional low-angle peak at $2 \theta=9^{\circ}$ that does not have a counterpart in the simulation. A possible explanation is that the sample was contaminated by a small amount of the powder $\mathbf{5 b}$ (see Experimental Section, main article).

Data for $\mathbf{4}$ are not included, since a bulk sample of that compound was not obtained for analysis. 


\section{References}

(1) Turner, C. J.; Cheeseman, G. W. H. ${ }^{13}$ C N.M.R. Spectra of 2-Substituted Pyrimidines. Org. Magn. Reson. 1976, 8, 357-360.

(2) Guionneau, P.; Marchivie, M.; Bravic, G.; Létard, J.-F.; Chasseau, D. Structural Aspects of Spin Crossover. Example of the $\left[\mathrm{Fe}^{\mathrm{II}} \mathrm{L}_{\mathrm{n}}(\mathrm{NCS})_{2}\right]$ Complexes. Top. Curr. Chem. 2004, 234, 97-128.

(3) O'Keeffe, M. The Prediction and Interpretation of Bond Lengths in Crystals. Struct. Bonding (Berlin) 1989, 71, 161-190.

(4) Brown, I. D. https://www.iucr.org/resources/data/data-sets/bond-valence-parameters

(5) (a) Létard, J.-F. Photomagnetism of Iron(II) Spin Crossover Complexes - the T(LIESST) Approach. $J$. Mater. Chem. 2006, 16, 2550-2559.

(b) Chastanet, G.; Desplanches, C.; Baldé, C.; Rosa, P.; Marchivie, M.; Guionneau, P. A Critical Review of the T(LIESST) Temperature in Spin Crossover Materials - What it is and What it is Not. Chem. Sq. 2018, 2, 2/1-19.

(6) O'Connor, C. J. Magnetochemistry - Advances in Theory and Experimentation. Prog. Inorg. Chem. 1982, 29, 203-283.

(7) (a) Hauser, A. Light-Induced Spin Crossover and the High-Spin $\rightarrow$ Low-Spin Relaxation. Top. Curr. Chem. 2004, 234, 155-198.

(b) Wenger, O. S. Is Iron the New Ruthenium? Chem. Eur. J. 2019, 25, 6043-6052.

(8) Öhrströhm, L.; Larsson, K. Molecule-Based Materials - the Structural Network Approach, Elsevier, Amsterdam, 2005, p. 314. 